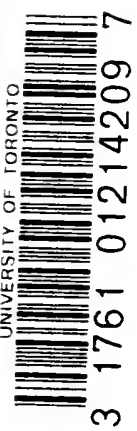









\section{LECONS}

SUR LES

FONCTIONS ENTIÈRES. 


\section{DU MÉME AUTEUR.}

Leçors sur ha TuLionie des foxctioxs. Éléments de la Théorie des ensembles et applications. (Paris, Gauthier-Villars et fils, 1898 . - Prix : 3 fr. 5o.)

\section{EN PREPARATION.}

Nouvelles Leçons sur li Théorje des foxctions. Leçons sur les séries divergentes. 
NOUVELLES LEC̣ONS SUR LA THÉORIE DES FONCTIONS.

\section{LECONS}

SCR LES

\section{FONCTIONS ENTIERES}

P.II

\section{ÉMILE BOREL,}

MAITRE DE CONFÉRENCES A L'ÉCOLE NorMale SUPÉRIEURE.

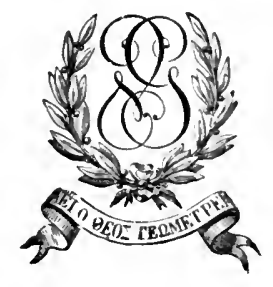

\section{PARIS,}

GAUTHIER-VILLARS, IMPRIMEUR-LIBRAIRE

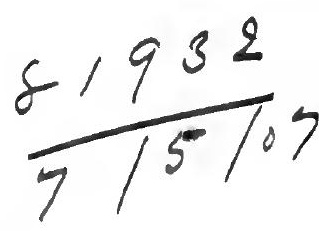

De L'obsebvatoire de paris et du bureau des longitudes,

Quai des Grands-Augustins, 55.

\section{0}

(Tous droits réservés.) 


$$
\begin{aligned}
& \text { QA } \\
& 351 \\
& B 67
\end{aligned}
$$




\section{PRÉFACE.}

Ce petit Livre a été rédigé d'après des Leçons faites ì l'École Normale pendant l'année scolaire $1897-1898$ ('). Ces Leçons s'adressaient aux élèves de seconde année, c'està-dire à des jeunes gens dont les connaissances en Analyse sont généralement peu étendues, mais solides; la plupart d'entre eux ne connaissent que le programme de la Licence $\left({ }^{2}\right)$. mais presque tous le possèdent bien. Il est dès lors possible, après avoir choisi un sujet bien délimité, d'aller assez vite et d'arriver en peu de leçons à approcher, au moins sur certains points, des limites actuelles de la science. On montre ainsi, sur un exemple particulier tout au moins, quelle est la nature des méthodes employées dans la recherche mathématique et quelle est la forme sous laquelle se posent les problèmes qui restent à résoudre.

Cette conception de l'enseignement me conduit à publier sur la Théorie des fonctions une série de petits livres, dont voici le second et qui seront, en principe, complètement indépendants les uns des autres. J'entends par là que chacun d'eux pourra être lu par un lecteur pourvu seulement des connaissances générales que je rappelais il y a un instant. Mais j'espère que l'ensemble de ces livres pourra néanmoins

( $\left.{ }^{1}\right)$ Je dois remercier deux de mes élèves, MM. Dubesset et Genty, dont les notes m'ont été fort utiles pour cette rédaction.

( ${ }^{2}$ En ce qui concerne la théorie des fonctions, qui nous occupe surtout ici, ce programme correspond à peu près aux parties de cette théorie développées dans le Cours autographié de M. IIermite. 
être considéré comme formant un tout, car ils seront écrits dans le même esprit et inspirés par les mêmes idées directrices.

Je dirai peu de chose sur le sujet même de ce Livre; l'Index placé ci-contre montre comment on peut marquer par quelques noms les progrès successifs faits depuis une vingtaine d'années dans la théorie des fonctions entières : je suis ainsi dispensé d'écrire un historique.

J'ai rejeté dans des Notes quelques développements d'une nature moins élémentaire que ceux du texte et aussi certaines considérations, en partie nouvelles, que j'ai dù me contenter d'esquisser brièvement, car leur champ d'application me paraît dépasser notablement les limites de la théorie qui est l'objet propre de ce Livre. 


\section{INDEX.}

Chap. I. - Le théorème fondamental de IVeierstrass ...... I Chap. II. - Les idées de Laguerre ................ 24

Chap. III. - Les inégalités de M. Poincaré............ 48

Chap. IV. - Les résultats de M. Hadamard ...........

Chis. V. - Le théorème de M. Picard............... 88

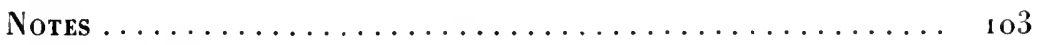

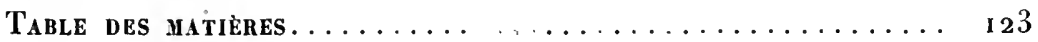

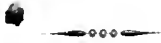





\title{
LEC CONS
}

SUR LES

\section{FONCTIONS ENTIERES.}

\author{
CHAPITRE I. \\ LE THÉORĖME FONDAMENTAL DE WEIERSTRASS.
}

Généralités sur les fonctions entières.

On appelle fonction entière une fonction analytique n'admettant aucune singularité à distance finie ( 1 ). Une telle fonction est caractérisée par le fait que son développement taylorien a un rayon de convergence infini. L'étude des fonctions entières peut ainsi ètre considérée comme une introduction à l'étude générale des fonctions définies par un développement de Taylor.

En réalité, une étude tant soit peu approfondie de ces diverses questions montre vite que les difficultés réclles y sont les mêmes et sont très grandes. Néanmoins, certains problèmes, bien particuliers sans doute, mais dont la solution peut rendre de très grands services dans les applications, se traitent plus aisément sur les fonctions entières. Il est donc assez naturel de les étudier tout d'abord, avant de s'occuper des fonctions analytiques les plus générales.

Étant donnée une fonction entière

$$
\mathrm{F}(z)=a_{0}+a_{1} z+a_{2} z^{2}+\ldots+a_{m} z^{m}+\ldots,
$$

(1) Certains auteurs emploient la locution fonction transcendante entière, réservant l'expression de fonction entière pour désigner les polynomes. Mais ce dernier mot est assez clair et assez communément adopté, pour qu'il nous paraisse absolument inutile de Iui créer un synonyme; il n'y a dés lors aucun inconvénient à dire simplement fonction entière au lieu de fonction transcendante entière. 
la question qui se pose tout d'abord est la suivante : comment varie $\mathbf{F}(z)$ lorsque $z$ se déplace dans son plan? Si l'on savait résoudre simplement cette question pour tous les déplacements possibles de $z$, on pourrait dire que l'on connaît parfaitement la fonction $F(z)$. C'est ainsi que nous connaissons bien les fonctions $e^{z}, \cos z, \sin z, \sigma z$ et quelques autres. On ne peut espérer arriver à étudier aussi complètement une fonction entière donnée par un développement en série absolument quelconque tel que (1); l'étude d'un tel développement peut conduire néanmoins à d'importants résultats, qui seront exposés au cours de ces Leçons.

La première proposition générale sur le développement (r) est due à Cauchy : elle consiste en ce que $\mathrm{F}(z)$ ne peut pas rester fini sans se réduire à une constante. Plus généralement, s'il existe un nombre $m$ tel que (sauf au voisinage de $z=0$ ) le quotient $\frac{\mathrm{F}(z)}{z^{m}}$ soit inférieur en module à un nombre fixe $\mathrm{M}$, on peut affirmer que $\mathrm{F}(z)$ se réduit à un polynome de degré ma plus.

En effet, si nous divisons les deux membres dé l'égalité (I) par $z^{m+q+1}$ et si nous intégrons le long d'un cercle C ayant son centre à l'origine, nous obtenons

$$
\int_{c} \frac{\mathrm{F}(z) d z}{z^{m+q+1}}=2 i \pi a_{m+q}
$$

car l'intégrale de tous les autres termes du second membre est nulle le long d'un contour fermé. Or, la longueur du contour d'intégration est $2 \pi \mathrm{R}$, si l'on désigne par $\mathrm{R}$ le rayon du cercle $\mathrm{C}$; comme le module de $\mathbf{F}(z)$ est inférieur à $\mathrm{MR}^{m}$, on en conclut que le module du premier membre est inférieur à $\frac{2 \pi \mathrm{M}}{\mathrm{R} q}$ et, par suite, que l'on a, quel que soit $\mathrm{R}$,

$$
\left|a_{m+q}\right|<\frac{\mathrm{M}}{\mathrm{R} q} .
$$

11 en résulte $\alpha_{m+q}=0$, pour toute valeur positive de $q$. C. Q. F. D.

On peut, comme l'a montré M. Hadamard ('), obtenir par une 
méthode analogue un résultat plus complet. Posons

$$
\begin{gathered}
z=r(\cos \theta+i \sin \theta), \\
a_{m}=x_{m}+i \beta_{m}, \\
\mathrm{~F}(z)=\mathrm{P}(r, \theta)+i \mathrm{Q}(r, 0) ;
\end{gathered}
$$

nous aurons, en séparant le réel de l’imaginaire,

$$
\begin{aligned}
P(r, \theta)=\alpha_{0} & +\left(\alpha_{1} \cos \theta-\beta_{1} \sin \theta\right) r+\ldots \\
& +\left(\alpha_{m} \cos m \theta-\beta_{m} \sin m \theta\right) r^{m}+\ldots
\end{aligned}
$$

On en conclut, par un procédé connu ('),

$$
\begin{gathered}
2 \pi \alpha_{0}=\int_{0}^{2 \pi} \mathrm{P}(r, 0) d \theta, \\
\pi r^{m} \alpha_{m}=\int_{0}^{2 \pi} P(r, \theta) \cos m \theta d \theta, \quad m \neq 0, \\
\pi r^{m} \beta_{m}=-\int_{0}^{2 \pi} P(r, 0) \sin m \theta d \theta, \quad m \neq 0 .
\end{gathered}
$$

Les deux dernières égalités donnent d'ailleurs, en remarquant que $\alpha_{m}+i \beta_{m}=a_{m}, \cos m \theta-i \sin m \theta=e^{-i m \theta}$,

$$
\pi r^{m} a_{m}=\int_{0}^{2 \pi} \mathrm{P}(r, 0) e^{-i m \theta} d \theta \text {. }
$$

On en conclut, le module du facteur $e^{-i m \theta}$ étant égal à l'unité,

$$
\pi r^{m}\left|a_{m}\right| \leqq \int_{0}^{2 \pi}|\mathrm{P}(r, 0)| d 0 .
$$

Les relations (2) et (3) donnent enfin, par addition et soustraction,

$$
\begin{aligned}
& \pi r^{m}\left|a_{m}\right|+2 \pi x_{0} \leqq \int_{0}^{2 \pi}[|\mathrm{P}(r, 0)|+\mathrm{P}(r, \theta)] d \theta \\
& \pi r^{\prime \prime}\left|a_{m}\right|-2 \pi x_{0} \leqq \int_{0}^{2 \pi}[|\mathrm{P}(r, 0)|-\mathrm{P}(r, \theta)] d \theta .
\end{aligned}
$$

Considérons, par exemple, la première de ces deux inégalités; la quantité à intégrer est visiblement nulle lorsque $\mathrm{P}$ est négatif, et

(') Ce procédé, devenu classique depuis les travaux de Fourier sur les séries trigonométriques, consiste à intégrer entre o et $2 \pi$ les deux membres de l'égalité (1), multipliée successivement par $\mathbf{r}, \cos \theta, \sin \theta, \ldots, \cos m \theta, \sin m \theta, \ldots$ 
égale à $2 \mathrm{P}$ lorsque $\mathrm{P}$ est positif. Si donc nous désignons par $\mathrm{A}(r)$ le maximum des valeurs positives de $\mathrm{P}(r, \theta)$ lorsque, $r$ étant constant, $\theta$ varie de o à $2 \pi$, nous avons

$$
\pi r^{m}\left|a_{m}\right|+2 \pi \alpha_{0} \leqq 4 \pi \mathrm{A}(r) .
$$

De même, en désignant par $\mathrm{B}(r)$ le maximum des valeurs positives de $-\mathrm{P}(r, 0)$ pour $r$ constant et $\theta$ variable, la seconde des inégalités donnera

$$
\pi r^{m}\left|a_{m}\right|-2 \pi x_{0} \leqq 4 \pi \mathrm{B}(r) .
$$

Lcs inégalités (4) et (5) nous seront fort utiles dans la suite. Pour le moment, nous voulons simplement attirer l'attention sur ce fait que le second membre de (4), par exemple, ne dépend que des valcurs posilives de la partie réelle de $\mathrm{F}(z)$. Si donc on suppose que la partie réelle de $\mathrm{F}(z)$ soit toujours algébriquement inférieure ( ${ }^{1}$ ) à $M r q, r$ désignant le module de $z$, M et $q$ des nombres positifs fixes, on en conchura que $a_{m}$ est nul pour $m>q$, c'est-à-dire que $\mathrm{F}(z)$ se réduit à un polynome. Ainsi, lorsque $\mathrm{F}(\tilde{z})$ n'est pas un polynome, on peut affirmer, non seulement que son module dépasse tout nombre assignable, mais encore que sa partie réelle $\mathrm{P}(r, \theta)$ [et naturellement aussi $\mathrm{Q}(r, \theta)$ ] prend des valeurs, soit positives, soit négatives, supérieures en valeur absolue à tout nombre donné, et même à $M r^{q}$, quels que soient les nombres fixes M el $q$.

Dans l'ordre d'idées où nous nous trouvons en ce moment, nous devons signaler un important théorème de Weierstrass, que nous nous contenterons de rappeler brièvement, car nous n'en aurons pas besoin el nous allons obtenir dans un instant un résultat plus complet, mais, il cst vrai, moins aisé à démontrer. Cette proposition est la suivante: Dans le voisinage d'un point singulier essentiel, une fonction uniforme peut approcher autant que l'on veut de toute valeur donnée. En particulier, une fonction cntière $\mathrm{F}(z)$ peut devenir aussi voisine que l'on veut d'un nombre quelconque $a$ donné à l'avance $\left({ }^{2}\right)$.

( ${ }^{1}$ Elle peut ainsi avoir des valeurs négatives très grandes en valeur absolue; on ne suppose rien à leur égard.

$\left({ }^{2}\right)$ La démonstration est fondée sur la considération de la fonction $\frac{1}{F(z)-a}$ 
L'étude de ce théorème de Weierstrass a conduit M. Picard à se poser la question suivante : l'équation

$$
\mathbf{F}(z)=a
$$

a-t-elle effectivement des racines, quel que soit $a$ ? Le dernier Chapitre de ces Leçons est consacré à l'étude détaillée de cette question, et de questions qui s'y rattachent; mais il n'est pas sans intérêt d'indiquer dès maintenant un résultat important obtenu par M. Picard dès $1880\left({ }^{1}\right)$.

Considérons d'abord une fonction entière $F(z)$ telle que l'équation

$$
\mathrm{F}(z)=0
$$

u'ait pas de racines, et posons

$$
\mathrm{G}(z)=\log \mathrm{F}(z)
$$

La fonction $\mathrm{G}(z)$ est manifestement régulière en tout point du plan, puisque $\mathrm{F}(z)$ n'est jamais nul ni infini; c'est donc une fonction entière; on a ainsi

$$
\mathbf{F}(z)=e^{\mathbf{G}(z)}
$$

telle est la forme d'une fonction entière qui n'a pas de zéros $\left({ }^{2}\right)$.

Considérons maintenant une fonction entière $F(z)$ telle que les équations

$$
\begin{aligned}
& \mathrm{F}(z)=0, \\
& \mathrm{~F}(z)=1,
\end{aligned}
$$

n'aient pas de racines $\left({ }^{3}\right)$, et désignons par $\varpi(x)$ la fonction modulaire, c'est-à-dire la fonction qui exprime au moyen du module

qui, si elle ne devient pas une infinité de fois infinie, est visiblement égale au quolient d'une fonction entière par un polynome et peut dépasser, par suite, tout nombre assignable.

(') Annales de l'École Normale, $\mathbf{1} 880$ (S. II, T. IX).

(2) Suivant un usage assez répandu, nous appelons zéros d'une fonction lcs racines de l'équation obtenue en égalant cette fonction à zéro.

$\left({ }^{3}\right)$ Si l'on a une fonction $F_{1}(z)$ telle que les équations $F_{1}(z)=a, F_{1}(z)=b$ n'aient pas de racines, on posera

$$
F(z)=\frac{F_{1}(z)-a}{b-a} .
$$


le rapport des périodes d'une fonction elliptique ( 1 ); on sait que la fonction $\varpi(x)$ n'admet que les points singuliers $0,1, \infty$; et, d'autre part, que le coefficient de $i$ dans $\varpi(x)$ est constamment de même signe; on pent, par cxemple, le supposcr positif. Dès lors, considérons, avec M. Picard, la fonction $\varpi[\mathrm{F}(z)]$; ce sera une fonction analytique régulière en tout point à distance finie; c'est donc une fonction entière $\left({ }^{2}\right)$. D'après une remarque faite il y a un instant, cette fonction doit se réduire à une constante, puisque sa partie imagivaire est constamment positive; le maximum des valeurs négatives de $-\mathrm{Q}(r, \theta)$ est ici zéro. Ainsi se trouve démontré le premier théorème de M. Picard: Une fonction entière $\mathrm{F}(z)$, telle que les équations

$$
\left\{\begin{array}{l}
\mathrm{F}(z)=a, \\
\mathrm{~F}(z)=b, \quad a \neq b
\end{array}\right.
$$

n'aient pas de racines, se réduit nécessairement à une constante. Nous n'utiliserons d'ailleurs pas ce théorème; nous le retrouverons par une voie directc, c'est-à-dire sans faire appel à la théorie des fonctions modulaires $\left({ }^{3}\right)$. Mais nous avons tenu, à cause de sa brièveté et de son élćgance, à rappeler ici la démonstration même de M. Picard. Rappelons aussi qu'avec de légères modificakions la même méthode a permis à M. Picard de démontrer que siles équations (6) ont tontes deux un nombre limité de racines, la fonction $F(z)$ se réduit à un polynome. Nous généraliserons plus loin ce résultat par la voie directe.

Une dernière remarque relativement aux fonctions entières qui n'ont pas de racines. Nous avons vu qu'elles sont nécessairement de la forme

$$
\mathbf{F}(z)=e^{\mathbf{G}(z)},
$$

(') On pose, par exemple, $\mathrm{J}=\int_{0}^{u} \frac{d u}{\sqrt{u(1-u)(1-k u)}}$ et l'on regarde le rapport de deux périodes primitives de cette intégrale comme une fonction de $k$.

( ${ }^{2}$ ) Ici, comme précédemment pour le logarithme, il est absolument inutile de démontrer que la fonction est uniforme, puisqu'clle est régulière en tout point z du plan. Le cercle de convergence du développement de Taylor de cette fonction a donc nécessairement un rayon infini.

$\left({ }^{3}\right)$ Voir le Chapitre V et la note I. 
$\mathrm{G}(z)$ étant une fonction entière (ou un polynome). Si G(z) n’est pas un polynome, nous savons que la partie réelle de $\mathrm{G}(z)$ devient, quels que soient $M$ et $q$, algébriquement supérieur à $M r^{q}, r$ désignant le module de $z$. Donc le module de $\mathrm{F}(z)$ devient supérieur à $e^{\mathrm{M} r}$. La fonction $\mathrm{F}(z)$ prend donc des valeurs bien plus grandes que dans le cas où $\mathrm{G}(z)$ est un polynome de degré quelconque, mais déterminé. Si, comme il est naturel, et cette induction sera confirmée par tout ce qui suit, on regarde une fonction entière comme d'autant plus simple qu'elle croît moins vite avec $z$. on voit que, parmi les fonctions entières qui n'ont pas de zéros, la plus simple (') est la fonction $e^{z}$.

On voit que l'étude approfondie de la théorie générale des zéros des fonctions entières aurait nécessairement conduit à introduire la fonction exponentielle et à lui donner une place distinguée en Analyse, si elle n'avait déjà occupé cette place depuis un siècle. C'est là un fait très fréquent : les cas particuliers intéressants sont plus souvent trouvés sans méthode bien définie qu'à l'aide des théories générales; cela ne doit pas nous empêcher de cultiver ces dernières.

\section{Les facteurs primaires.}

On sait qu'un polynome $\mathbf{P}(z)$ de degré $m$ est caractérisé par le fait qu'il a $m$ zéros. Si l'on donne ces $m$ zéros : $a_{1}, a_{2}, \ldots, a_{m}$ (distincts ou non), on a, en désignant par A une constante,

$$
\mathrm{P}(z)=\mathrm{A}\left(z-a_{1}\right)\left(z-a_{2}\right) \ldots\left(z-a_{m}\right) .
$$

Le polynome est ainsi déterminé, à un facteur constant près, par la connaissance de ses zéros, lesquels pourraient être donnés arbitrairement a priori.

Ces remarques bien simples ont-elles des analogues dans lat théorie des fonctions entières? Le but de ce paragraphe est d'indiquer la réponse donnée par Weierstrass à cette question.

Observons d'abord que, dans une aire finie, une fonction en-

( $\left.{ }^{1}\right)$ Il est clair que l'on pourrait considérer $e^{a z}$ et prendre pour $a$ des valeurs de plus en plus petites; mais cela n'a aucun intérèt. 
tière $\mathrm{F}(z)$ possède néeessairement un nombre fini de zéros. Car, si les zéros n’étaient pas en nombre limité, leur ensemble posséderait nécessairement un point limite $A$, c'est-à-dire qu'il y aurait une infinité de zéros dans un cercle aussi petit que l'on veut, ayant son centre en A. Ce résultat est en contradiction avec l'hypothèse que la fonction est régulière en $\mathrm{A}$.

Les zéros de $\mathrm{F}(z)$ étant en nombre limité dans toute aire finie, il est possible de les supposer rangés d'après l'ordre de grandeur de leurs modules; si plusieurs ont le même module, on pourra leur donner un ordre arbitraire. Nous désignerons les zéros par

$$
a_{1}, a_{2}, \ldots, a_{m}, \ldots,
$$

et nous poserons $\left|a_{m}\right|=r_{m}$; nous aurons, par hypothèse,

$$
r_{1} \leqq r_{2} \leqq r_{3} \leqq \cdots \leqq r_{m} \leqq \cdots
$$

D’ailleurs, les zéros ne sont pas néeessairement distinets; mais les zéros multiples figurent dans la suite des $a$ un nombre de fois égal à leur degré de multiplicité; on peut avoir $a_{1}=a_{2}=a_{3}$; $a_{4}=a_{3} ; \ldots$, le point $z=a_{1}$ est alors un zéro triple, $z=a_{4}$ un zéro double, ete.

Nous supposerons $a_{1} \neq 0$; si la fonction donnée s'annulait pour $\approx=0$, il suffirait de mettre en facteur une puissance de $z$ pour être ramené au cas que nous traitons.

Supposons d'abord que la série à termes positifs

$$
\frac{1}{r_{1}}+\frac{1}{r_{2}}+\ldots+\frac{1}{r_{m}}+\cdots
$$

soit convergente $\left(^{1}\right)$. Dans ce cas, le produit infini

$$
\Pi(z)=\left(1-\frac{z}{a_{1}}\right)\left(1-\frac{z}{a_{2}}\right) \cdots\left(1-\frac{z}{a_{m}}\right) \cdots
$$

( $\left.{ }^{1}\right)$ Ce cas comprend celui où les zéros sont en nombre limité; si Jeur nombre est $m$, la série (2) se réduit à une somme de $m$ termes. On doit alors supposer que $r_{n+q}$ est infini pour $q=1,2, \ldots$ Cette hypothèse s'accorde bien avec ce qui se passe pour les polynomes; lorsqu'un certain nombre de racines disparaissent, lorsque, par exemple, un polynome à coefficients variables de degré $m+q$ n'a que $m$ racines, cela tient à ce que $q$ racines ont augmenté indéfiniment, et l'on dit qu'il y a $q$ racines infinies. 
est absolument convergent pour toute valeur de z et uniformément convergent dans tout domaine limité. En effet, si l'on a $|z| \leqq r$, pour prouver que le produit $\Pi(z)$ est absolument et uniformément convergent, il suffit de constater la convergence du produit infini

$$
\left(\mathrm{I}+\frac{r}{r_{1}}\right)\left(\mathrm{I}+\frac{r}{r_{2}}\right) \cdots\left(\mathrm{I}+\frac{r}{r_{m}}\right) \cdots
$$

et cette convergence résulte, comme on sait, de la convergence de la série (2).

Le produit $\Pi(z)$ représente donc une fonction entière, qui admet les mèmes zéros que $\mathrm{F}(z)$; le quotient $\frac{\mathrm{F}(z)}{\mathrm{II}(z)}$ est donc aussi une fonction entière (1). Ce quotient ne pourrait, en effet, admettre comme point singulier à distance finic que les zéros de II $(z)$, et l'on voit immédiatement qu'il est régulier en ces points; d'ailleurs il ne saurait y être nul. C'est donc une fonction entière dépourvue de zéros; on en conclut que l'on a

$$
\frac{\mathbf{F}(z)}{\boldsymbol{\Pi}(z)}=e^{\mathbf{G}(z)}
$$

$\mathrm{G}(z)$ étant une fonction entière (qui, dans des cas particuliers, pourrait se réduire à un polynome ou même à une constante). On a donc finalement

$$
\mathbf{F}(\boldsymbol{z})=e^{\mathbf{G}(z)} \boldsymbol{\Pi}(\boldsymbol{z}),
$$

c'est-à-dire

$$
\mathrm{F}(z)=e^{\mathbf{G}(z)}\left(1-\frac{z}{a_{1}}\right)\left(1-\frac{z}{a_{2}}\right) \cdots\left(1-\frac{z}{a_{m}}\right) \cdots
$$

Nous renvoyons les remarques qu'appelle cet important résultat après l'étude du cas général que nous allons maintenant aborder. Ce cas est celui où l'on ne suppose rien sur la convergence de la série (2). On sait seulement que $r_{n}$ augmente indéfiniment avec $n$. Il en résulte que l'on peut trouver une série de nombres entiers positifs

$$
\rho_{1}, \rho_{2}, \ldots, \rho_{n}, \ldots,
$$

( ') Il est à peine utile d'observer que chaque zéro multiple a été introduit avec son degré de multiplicité. 
tels que la série à termes positifs

$$
\left(\frac{r}{r_{1}}\right)^{p_{1}}+\left(\frac{r}{r_{2}}\right)^{p_{2}}+\ldots+\left(\frac{r}{r_{n}}\right)^{p_{n}}+\ldots
$$

converge quel que soit $r$. C'est ce qui a lien en tous cas, comme l'a remarqué Weierstrass, si l'on prend

$$
\rho_{n}=n \text {. }
$$

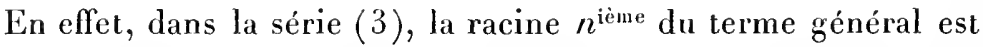
alors $\frac{r}{r_{n}}$; elle tend vers zéro pour $n$ infini. On peut observer qu'il suffit de prendre

$$
\rho_{n}=\mathrm{E}(\log n)
$$

en désignant par $\mathbf{E}(x)$ la partie entière de $x$.

On a, en effet,

$$
\left(\frac{r}{r_{n}}\right)^{\log n}=e^{\left(\log r-\log r_{n}\right) \log n}=n^{\log r-\log r_{n}}
$$

et, $r_{n}$ augmentant indéfiniment avec $n$, les termes de la série (3) sont, à partir d'un certain rang, inférieurs à ceux de la série

$$
\Sigma n-q \text {, }
$$

quel que soit le nombre fixe $q$. La série (3) est donc convergente $(1)$.

Observons enfin que, s'il existe un nombre entier $p$ tel que la série

$$
\sum \frac{\mathrm{I}}{r_{n}^{p}}
$$

soit convergente, on peut prendre

$$
\rho_{1}=\rho_{2}=\ldots=\rho_{n}=\ldots=p \text {. }
$$

Mais, pour l'instant, il importe peu de connaître la valeur des $p_{n}$;

( ${ }^{1}$ Un calcul analogue montre aisément que, comme je l'ai indiqué dans les $\operatorname{Acta}(\mathrm{t} . \mathrm{XX}, \mathrm{p} .360)$, il suffit de prendre $\rho_{n} \equiv \mathbf{E}\left(\frac{2 \log n}{\log r_{n}}\right) \cdot$ Le nombre 2 pourrait d'ailleurs ètre remplacé par un autre nombre quelconque supérieur à un. 
il suffit d'avoir constaté qu'ils peuvent toujours ètre choisis de manière à assurer la convergence de la série $(3)$.

Nous appellerons facteur. primaire (1) de genre $\left({ }^{2}\right) k$ l'expression

$$
\mathrm{P}_{k}(u)=(1-u) e^{\frac{u}{1}+\frac{u^{2}}{2}+\frac{u^{3}}{3}+\cdots+\frac{u^{k}}{h}} .
$$

On voit que l'exposant de $e$ est formé par les $k$ premiers termes du développement en série de $\log \frac{1}{1-u}$. C'est là l'idéc fondamentale de Weierstrass d'où découlent, comme on va le voir, les propriétés essentielles de $\mathrm{P}_{k}(u)$.

Nous allons étudier le développement en série de $\mathrm{P}_{k}(u)$ suivant les puissances croissantes de $u$. On a

$$
\begin{aligned}
\log \mathrm{P}_{k}(u) & =\log (1-u)+\frac{u}{\mathrm{i}}+\frac{u^{2}}{2}+\ldots+\frac{u^{k}}{k} \\
& =-\frac{u^{k+1}}{k+1}-\frac{u^{k+2}}{k+2}-\frac{u^{k+3}}{k+3}-\ldots
\end{aligned}
$$

Donc

$$
\mathrm{P}_{k}(u)=e^{-\frac{u^{k+1}}{k+1}-\frac{u^{k+2}}{k+2}-\cdots}=\mathbf{I}+\beta_{1} u^{k+1}+\beta_{2} u^{k+2}+\cdots
$$

Ainsi le terme indépendant de $u$ est égal à l'unitć et les coefficients des $k$ premières puissances de $u$ sont nuls. Il est clair, d'ailleurs, que $\mathrm{P}_{k}(u)$ étant une fonction entière, le résultat obtenu est valable quel que soit $u$, bien que les séries employées dans les calculs intermédiaires ne soient convergentes que lorsque le module de $u$ est inférieur à l'unité.

Nous allons obtenir maintenant des inégalités importantes auxquelles satisfont les coefficients $\beta$. Considérons l'expression $e^{\frac{u}{1}+\frac{u^{2}}{2}+\ldots+\frac{u^{k}}{u}}=\mathrm{I}+\left(\frac{u}{1}+\frac{u^{2}}{2}+\ldots+\frac{u^{k}}{k}\right)+\frac{1}{1 \cdot 2}\left(\frac{u}{1}+\frac{u^{2}}{2}+\ldots+\frac{u^{k}}{k}\right)^{2}+\ldots$

( $\left.{ }^{1}\right)$ Weierstrass a donné le nom de facteur primaire à toute expression de la forme

$$
(k z+l) e^{\mathrm{G}(z)} \text {; }
$$

c'est la fonction entière la plus générale ne possédant qu'un zẻro (ce zẻro disparait mème si $k$ est nul). Au problème de la décomposition d'un polynome en facteurs du premier degré (ou polynomes à un seul zéro) correspond celui de la décomposition d'une fonction entière en facteurs primaires (ou fonctions entières à un seul zéro).

(2) Comme nous le verrons dans le Chapitre suivant, c'est Laguerre qui a introduit cette dénomination. 
Si on l'ordonne suivant les puissances de $u$, on obtiendra une série dont tous les coefficients seront positifs; il est manifeste que ces coefficients augmentent si l'on augmente le nombre $k$; car chacun d'eux est la somme de termes tous positifs, et dont le nombre augmente avec $k$.

Or, si le nombre $k$ augmente indéfiniment, on obtient

$$
e^{\frac{u}{1}+\frac{u^{2}}{2}+\ldots+\frac{u^{k}}{k}+\ldots}=e^{\log \frac{1}{1-u}}=\frac{1}{1-u}=\mathrm{r}+u+u^{2}+u^{3}+\ldots,
$$

c'est-à-dire que tous les coefficients deviennent égaux à l'unité('). Il en résultc que si l'on pose

$$
e^{\frac{u}{1}+\frac{u^{2}}{2}+\ldots+\frac{u^{k}}{k}}=1+\alpha_{1} u+\alpha_{2} u^{2}+\ldots+\alpha_{n} u^{n}+\ldots
$$

on aura, quel que soit $u$,

$$
0<\alpha_{n}<1 .
$$

Or, par définition,

$\mathrm{P}_{k}(u)=(1-u) e^{\frac{u}{1}+\frac{u^{2}}{2}+\ldots+\frac{u^{k}}{k}}=(\mathrm{I}-u)\left(\mathrm{1}+\alpha_{1} u+\alpha_{2} u^{2}+\ldots+\alpha_{n} u^{n}+\ldots\right)$.

On avait posé

$$
\mathrm{P}_{k}(u)=\mathbf{I}+\beta_{1} u^{k+1}+\beta_{2} u^{k+2}+\ldots
$$

On a done

$$
\beta_{r}=\alpha_{k+r}-\alpha_{k+r-1},
$$

et, dès lors, les inégalités

entraînent

$$
0<\alpha_{k+r}<\mathrm{I}, \quad 0<\alpha_{k+r-1}<\mathrm{I}
$$

$$
\left|\beta_{r}\right|<1 .
$$

( ${ }^{1}$ Il n'est pas inutile d'observer que, pour toute valeur finie de $k$, $e^{u} u^{u}+\frac{u^{2}}{2}+\ldots+\frac{u^{k}}{k}$ est une fonction entière; lorsque $k$ devient infini on obtient une fraction rationnelle $\frac{1}{I-u} \cdot$ Le développement en série de la fonction entière a pour limite le développement en série de la fraction rationnelle, en ce sens que chaque coefficient a pour limite (pour $k$ infini) le coefficient correspondant, et c'est lout ce qui nous importe ici; mais la fonction entière ne tend pas, dans Lout le plan, vers la limite $\frac{\mathbf{I}}{\mathbf{I}-u}$. 
LE THÉORÈME FONDAMENTAL DE WEIERSTRASS.

Donc, si nous posons

$$
\mathrm{P}_{k}(u)=\mathrm{I}+\varphi_{k}(u) .
$$

nous aurons, en supposant le module de $u$ inférieur à l'unité,

$$
\begin{gathered}
\left|\wp_{k}(u)\right|<\left|\beta_{1} u^{k+1}\right|+\left|\beta_{2} u^{k+2}\right|+\ldots ; \\
\left|\wp_{k}(u)\right|<\left|u^{k+1}\right|+\left|u^{k+2}\right|+\ldots,
\end{gathered}
$$

c'est-à-dire

$$
\left|\varphi_{k}(u)\right|<\frac{\left|u^{k+1}\right|}{1-|u|} .
$$

Ces calculs préliminaires achevés, reprenons les nombres $a_{1}$, $a_{2}, \ldots, a_{n}, \ldots$ de modules $r_{1}, r_{2}, \ldots, r_{n}, \ldots$ et supposons les entiers $p_{n}$ choisis de manière que la série (3) soit eonvergente. Nous allons montrer que le produit infini

$$
\Pi(z)=\mathrm{P}_{\rho_{1}}\left(\frac{z}{a_{1}}\right) \mathrm{P}_{\rho_{z}}\left(\frac{z}{a_{2}}\right) \cdots \mathrm{P}_{\rho_{n}}\left(\frac{z}{a_{n}}\right) \cdots
$$

est absolument et uniformément convergent pour $|z|<r, r$ étant un nombre quelconque donné à l'avance. Il en résultera immédiatement que ce produit représente une fonction entière admettant les zéros $a_{1}, \ldots, a_{n}, \ldots$

Pour démontrer la convergence du produit infini, il suffit, puisque l'on a

$$
\mathrm{P}_{\rho_{n}}\left(\frac{\tilde{z}}{a_{n}}\right)=1+\varphi_{\rho_{n}}\left(\frac{z}{a_{n}}\right)
$$

de prouver la convergence de la série

$$
\sum\left|\varphi \dot{\rho_{n}}\left(\frac{\ddot{\dot{a}}}{a_{n}}\right)\right| .
$$

Pour démontrer celte convergence, nous pouvons négliger les termes, en nombre limité, pour lesquels le module de $a_{n}$ est inférieur à $r$. Pour chacun des autres termes, on peut écrire

$$
\left|i_{p_{n}}\left(\frac{z}{a_{n}}\right)\right|<\frac{\left(\frac{r}{r_{n}}\right)^{\rho_{n}}}{1-\frac{r}{r_{n}}},
$$

puisque le module de $a_{n}$ est égal à $r_{n}$ et le module de $z$ inférieur 
ou égal à $r$. La convergence absolue et uniforme de la série (4) est donc une conséquence immédiate ( ${ }^{1}$ ) de la convergence de la série à termes positifs

$$
\sum\left(\frac{r}{r_{n}}\right)^{p_{n}}
$$

Nous avons ainsi démontré le résultat fondamental qui est une des plus belles découvertes de Wejerstrass : Étant donnée une suite infinie quelconque de nombres dont le module croît indéfiniment (2), il est possible de former un produit de facteurs primaires, dont chacun s'annule pour un de ces nombres. Ce produit est d'ailleurs absolument et uniformément convergent dans tout domaine fini et représente par suite une fonction entière.

De ce résultat découlent deux conséquences principales:

1" Étant donné dans le plan un ensemble quelconque de points isolés $\left({ }^{3}\right)$, on peut former une fonction entière admettant ces points pour zéros.

On en conclut que le seul résultat général que l'on puisse énoncer relativement à la distribution des zéros d'une fonction entière quclconque, c'est que ce sont des points isolés : c'est la seule condition à laquelle ils soient assujettis. Cette remarque fait prévoir combien sont grandes les difficultés de toute recherche générale relative à ces zéros; elle fait comprendre pourquoi nous serons obligés de nous borner à des cas très particuliers en apparence, mais heureusement importants dans les applications.

(1) Il n'y a pas à s'arrêter à la présence du diviseur $\frac{1}{1-\frac{r}{r_{n}}}$ qui tend vers

l'unité lorsque $n$ augmente indéfiniment.

( $\left.{ }^{2}\right)$ Bien que nous ne nous occupions dans ces Leçons que des fonctions entières, nous pouvons faire observer, incidemment, que cette hypothèse n'a été utilisée que pour démontrer l'existence des $\rho_{n}$; or, si l'on suppose que les nombres $r_{n}$ croissent et tendent vers une limite $R$, il suffit de supposer $r<R$ et de prendre $\rho_{n}=n$ pour que la série ( 3 ) soit convergente; il n'y a dès lors rien à changer à nos calculs pour prourer que le produit infini obtenu converge absolument et uniformément à l'intérieur de tout cercle de rayon inférieur à $\mathbf{R}$. Mais le cercle de rayon $\mathrm{R}$ sera, en général, une ligne singulière essentielle pour la fonction, car on doit regarder comme le cas général celui où les $a_{n}$ ont pour points limites tous les points de la circonférence. (Voir Piciard, Traité d'Analyse.)

( $\left.{ }^{3}\right)$ C'est-d̀-dire n'ayant aucun point limite à distance finie. 
$2^{\circ}$ Étant donnée une fonction entière $\mathrm{F}(z)$, il existe un produit $\Pi(z)$ ayant les mêmes zéros; on a dès lors, en raisonnant comme plus haut,

$$
\mathbf{F}(z)=e^{\mathbf{G}(z)} \boldsymbol{\Pi}(z),
$$

$\mathrm{G}(z)$ étant une fonction entière. La fonction donnée $\mathrm{F}(z)$ se trouve décomposée en facteurs primaires; on peut dire aussi que l'on a l'expression générale des fonctions entières $\mathbf{F}(z)$, mais il faut observer que cette expression renferme une fonction entière arbitraire $G(z)$, c'est-à-dire un élément de même nature que celui que l'on cherche à définir. On peut cependant montrer que la fonction $\mathrm{G}(z)$ est, en général (1), plus simple que $\mathrm{F}(z)$, de sorte que l'on a bien fait un progrès dans la connaissance de $\mathbf{F}(z)$.

La détermination de ce facteur exponentiel $e^{\mathbf{G}(z)}$ est toujours la plus grande difficulté dans les applications du théorème fondamental de Weierstrass; nous reviendrons sur ce point dans le Chapitre IV, après avoir exposé les belles recherches de M. Наdamard.

Quelques renarques sur les séries à termes positifs.

Nous ne pouvons pas développer ici une théorie générale de la convergence des séries à termes positifs; ce sujet exigerait, à lui seul, un petit Livre; nous désirons seulement, sans revenir sur les résultats classiques que l'on trouve dans tous les Traités de Calcul différentiel, les compléter sur quelques points, dans la mesure qui est strictement indispensable pour la suite $\left({ }^{2}\right)$.

Les règles de convergence des séries à termes positifs s'obtiennent par la comparaison de la série proposée avec une série convergente connue,

$$
u_{1}+u_{2}+\ldots+u_{n}+\ldots
$$

( $\left.{ }^{1}\right)$ Il faut, pour cela, choisir convenablement les nombres $\rho_{n}$. [Voir mon Mémoire sur les zéros des fonctions entières (Acta mathematica, t. XX.)]

$\left({ }^{2}\right)$ Le lecteur trouvera des détails plus complets, ainsi que des renseignements bibliographiques, dans l'excellent article que M. Pringsheim a consacré à cette question dans l'Encyclopédie Burkhardt-Meyer. 
Si la série proposée est telle que son terme général soit inférieur à $u_{n}$, on peut affirmer sa convergence. De même, si la série (I) est divergente, toute série dont le terme général est supérieur à $u_{n}$ est sûrement divergente.

Mais il importe d'attirer l'attention sur le point suivant : Supposons que la série (1) soit divergente, mais que l'on ait cependant

$$
\lim _{n=\infty} u_{n}=0 .
$$

Je dis que l'on peut, dans ces conditions, trouver une série convergente

$$
v_{1}+v_{2}+\ldots+v_{n}+\ldots,
$$

telle que l'on ait, pour une infinité de valeurs de n,

$$
v_{n}>u_{n} .
$$

En effet, la condition (2) étant remplie, on peut déterminer une série d'indices successifs $n_{1}, n_{2}, \ldots, n_{p}, \ldots$, tels que l'on ait

$$
u_{n_{1}}<\frac{\mathrm{I}}{\mathrm{I}^{2}}, \quad u_{n_{\mathrm{z}}}<\frac{\mathrm{I}}{2^{2}}, \quad \ldots, \quad u_{n_{p}}<\frac{1}{p^{2}},
$$

Il suffira, dès lors, de prendre

$$
v_{n_{1}}=\frac{1}{1^{2}}, \quad v_{n_{2}}=\frac{1}{2^{2}}, \quad \ldots, \quad v_{n_{p}}=\frac{1}{p^{2}}, \quad \ldots
$$

pour que la condition (4) soit vérifiée pour une infinité de valeurs de $n$. La série (3) sera d'ailleurs convergente si l'on a soin, lorsque $n$ diffère de $n_{1}, n_{2}, \ldots, n_{p}, \ldots$, de prendre, par exemple,

$$
v_{n}=\frac{\mathrm{I}}{n^{2}} .
$$

Notre assertion est donc justifiée; c'est à des faits de ce genre qu'esı due la principale difficulté de l'étude des questions de convergence et de divergence, ou des questions connexes relatives aux modes de croissance des fonctions. C'est ce que l'on peut exprimer en disant que les modes de croissance peuvent ne pas ètre comparables. Nous ne pouvons nous étendre sur ces difficultés; notre but sera, au contraire, de les éviter le plus possible; cependant, il n'était pas inutile de les signaler, ne serait-ce 
LE THÉORÈME FONDAMENTAL DE WEIERSTRASS.

que pour bien faire voir que le théorème que nous allons maintenant démontrer n'est nullement évident.

Ce théorème est le suivant :

\section{Soit}

$$
u_{1}+u_{2}+\ldots+u_{n}+\ldots
$$

une série à termes posıtifs décroissants; si elle est convergente, on a

$$
\lim _{n=\infty} n u_{n}=0 .
$$

Il résulte, en effet, de ce qui précède que si les termes de la série proposée n'étaient pas décroissants, la proposition ne serait pas exacte.

Nous allons prouver que, si le produit $n u_{n}$ n'a pas pour limite zéro, la série proposée est forcément divergente. En effet, il existe alors un nombre positif $a$ tel que, pour une infinité de valeurs de $n$, l'on ait

$$
n u_{n}>a .
$$

Désignons ces valeurs de $n$ par

$$
n_{1}, n_{2}, \ldots, n_{p}, \ldots
$$

Nous pouvons toujours, en supprimant ('), s'il est nécessaire, un certain nombre de $n_{i}$, supposer que l'on a

$$
n_{2}>2 n_{1}, \quad n_{3}>2 n_{2}, \quad \ldots, \quad n_{p+1}>2 n_{p},
$$

c'est-à-dire

$$
n_{2} . \quad n_{1}>\frac{n_{2}}{2}, \quad n_{3}-n_{2}>\frac{n_{3}}{2}, \quad n_{p+1}-n_{p}>\frac{n_{p+1}}{2} .
$$

On a d'ailleurs, par hypothèse, $n_{i} u_{n_{i}}>a$, c'est-à-dire

$$
u_{n_{1}}>\frac{a}{n_{1}}, \quad u_{n_{3}}>\frac{a}{n_{2}}, \quad \ldots, \quad u_{n_{p+1}}>\frac{a}{n_{p+1}},
$$

La série proposée se compose d'une série de groupes de termes tels que le suivant :

$$
u_{n_{p}+1}+u_{n_{p}+2}+\ldots+u_{n_{p+1}} \text {. }
$$

( ${ }^{1}$ Les $n_{i}$ étant en nombre infini, il en existe un qui est supérieur à $2 n_{1}$; nous le désignerons par $n_{2}$; nous désignerons de mème par $n_{3}$ l'un des $n_{i}$ qui sont supérieurs à $n_{2}$, et ainsi de suite. Nous ne tiendrons pas compte des $n_{i}$ que nous n'aurons pas été amenés à considérer dans la suite indéfinie de ces opérations.

B. 
Or, les termes de la série étant décroissants, ce groupe de termes a une somme supérieure à

$$
\left(n_{p+1}-n_{p}\right) u_{n_{p+1}}>\frac{n_{p+1}}{2} \frac{a}{n_{p+1}}=\frac{a}{2} .
$$

La série, renfermant une infinité de groupes de termes dont la somme est supérieure à $\frac{a}{2}$, est nécessairement divergente.

\section{L'exposant de convergence.}

Nous pouvons maintenant étudier une notion fort importante dans la théorie des fonctions entières, celle de l'exposant de convergence (') d'une suite indéfinie de nombres positifs croissants

$$
r_{1}, \quad r_{2}, \ldots, r_{n}, \ldots
$$

Désignons par $\alpha$ un nombre positif quelconque el considérons la série

$$
\frac{\mathrm{I}}{r_{1}^{\alpha}}+\frac{1}{r_{2}^{\alpha}}+\ldots+\frac{\mathrm{I}}{r_{n}^{\alpha}}+\ldots
$$

Cette série peut être convergente ou divergente. Dans le premier cas nous dirons que le nombre $\alpha$ appartient à la première catégorie; dans le deuxième cas, qu'il appartient à la deuxième catégorie. Il résulte immédiatement des propriétés les plus élémentaires des séries à termes positifs que, si un nombre $\alpha^{\prime}$ appartient à la première catégorie, il en est de même de tout nombre supérieur à $\alpha^{\prime}$; si un nombre $\alpha^{\prime \prime}$ appartient à la deuxième catégorie, il en est de même de tout nombre inférieur à $\alpha^{\prime \prime}$.

Dès lors, on sait qu'il existe un nombre $p$ séparant les deux catégories, c'est-à-dire tel que la première catégorie soit formée des nombres plus grands que $\rho$ et la deuxième des nombres plus petits que $\rho$. Il peut d'ailleurs arriver, pour certaines suites (5), que l'une des deux catégories comprenne tous les nombres positifs; la valeur de $p$ est alors o ou $+\infty\left({ }^{2}\right)$.

Nous dirons que $p$ est l'exposant de convergence de la

(1) Voir la note de la page 26.

(2) Par exemple, si $r_{n}=n !, \rho=0$; si $r_{n}=\log n, \rho=\alpha$. 
suite (5); par définition, $\varepsilon$ étant un nombre positif quelconque, la série

$$
\sum \frac{\mathrm{I}}{r_{n}^{p+\varepsilon}}
$$

est convergente; et la série

$$
\sum \frac{1}{r_{n}^{p-\varepsilon}},
$$

est divergente; quant à la série

$$
\sum \frac{1}{r_{n}^{*}}
$$

elle peut être convergente ou divergente, de mème qu'une série de Taylor est, suivant les cas, convergente ou divergente pour un point appartenant à la circonférence de son cercle de convergence.

On voit immédiatement quelle relation existe entre la notion que nous venons d'introduire et la décomposition en facteurs primaires. Si nous supposons que la suite (5) est formée des modules des zéros d'une fonction entière, rangés dans l'ordre croissant, et, si l'exposant de convergence de la suite n'est pas infini, on pourra exprimer la fonction entière par le produit de facteurs primaires de genre fini, et d'un facteur exponentiel.

Mais nous reviendrons sur ce point dans le Chapitre suivant; nous voulons d'abord étudier en elle-mème la notion d'exposant de convergence d'une suite telle que $(5)$.

Nous venons de dire que la série

$$
\sum \frac{1}{r_{n}^{p+s}}
$$

est convergente; les termes de cette série sont d'ailleurs positifs et décroissants. Il résulte donc du théorème de la page i que l'on a

$$
\lim _{n=\infty} \frac{n}{r_{n}^{p+\varepsilon}}=0
$$

Donc, à partir d'une certaine valeur de $n$, l'on a

$$
\frac{n}{r_{n}^{p}+\varepsilon}<1
$$


c'est-à-dire

$$
r_{n}>n \frac{1}{\rho+\cdot \varepsilon}
$$

Si nous posons, pour abréger,

$$
\frac{\mathbf{I}}{\rho}=\lambda
$$

nous aurons, en désignant par $\eta$ un nombre positif,

$$
\frac{1}{\rho+\varepsilon}=\lambda-r
$$

de sorte que l'on peut écrire

$$
r_{n}>n \lambda-n
$$

Cette inégalité (6) est vérifiée, quel que soit le nombre positif $\eta$, à partir d'une certaine valeur de $n$. Nous pourrons exprimer ce fait en disant que $r_{n}$ croîl plus vite que $n^{\lambda-\eta}$.

Cherchons maintenant à utiliser le fait que la série

$$
\sum \frac{1}{r_{n}^{\rho-\varepsilon}}
$$

est divergente. Il en résulte que, $\varepsilon^{\prime}$ étant un nombre positif, on ne peut pas avoir, pour toutes les valeurs de $n$ dépassant un nombre donné,

$$
\frac{\mathrm{I}}{r_{n}^{p-\varepsilon}}<\frac{\mathrm{I}}{n^{1+\varepsilon^{\prime}}}
$$

on a donc, pour une infinité de valeurs de $n$,

$$
r_{n}^{p-\varepsilon}<n^{1+\varepsilon^{\prime}},
$$

c'est-à-dire

$$
r_{n}<n^{\lambda+n}
$$

$\eta$ étant un nombre positif quelconque.

Mais nous ne pouvons pas`affirmer que l'inégalité (7) ait lieu pour toutes les valeurs de $n$ supérieures à un nombre fixe, comme l'inégalité (6). Pour nous en rendre compte, désignons par $n_{1}$ un nombre entier quelconque et par $n_{2}, n_{3}, \ldots, n_{h}, \ldots$ des entiers assujettis seulement à vérifier les inégalités

$n_{2}^{2}>e^{n_{1}}, \quad n_{3}^{2}>e^{n_{2}+1}, \quad n_{4}^{2}>e^{n_{3}+1}, \quad \ldots, \quad n_{h+1}^{2}>e^{n_{h}+1}, \quad \ldots$ 
Prenons

$$
\begin{gathered}
r_{n_{1}}=e^{n_{1}}, \quad r_{n_{9}}=n_{2}^{2}, \quad r_{n_{3}+1}=e^{n_{2}+1}, \quad r_{n_{3}}=n_{3}^{2}, \quad \ldots . \\
r_{n_{l}}=n_{h}^{2}, \quad r_{n_{h}+1}=e^{n_{h}+1}, \quad r_{n_{h+1}}=n_{h+1}^{2}, \quad \ldots
\end{gathered}
$$

Il est clair que les nombres

$$
r_{n_{1}}, \quad r_{n_{3}}, \ldots, \quad r_{n_{h}}, r_{n_{h}+1}, r_{n_{h+1}}, \ldots
$$

vont en croissant avec leurs indices; $n$ étant compris entre $n_{h}+1$ et $n_{h+1}$, nous prendrons

$$
r_{n}=r_{n_{h+1}}+\frac{n_{h+1}-n}{n_{h+1}-n_{h}-1} r_{n_{h+1}},
$$

et nous aurons ainsi défini unè suite de nombres positifs croissants

$$
r_{1}, r_{2}, \ldots, r_{n}, \ldots,
$$

tels que l'on ait, pour une infinité de valeurs de $n$,

$$
r_{n}=n^{2},
$$

et pour une infinité de valeurs de. $n$,

$$
r_{n}=e^{n} \text {. }
$$

D'ailleurs, on constate aisément que: pour toute valeur de n, on a

$$
r_{n}>n^{2} .
$$

On en conclut que l'inégalité (6) est vérifiée pour toute valcur de $n$, si l'on prend $\lambda=2$. Elle cesserait de l'être pour une infinité de valeurs de $n$, si l'on prenait $\lambda>2($ et $n<\lambda-2)$. D'autre part, si l'on prend $\lambda<2$, l'inégalité $(7)$ ne sera vérifiée pour aucune valeur de $n$.

L'exposant de convergence de la suite (8) est donc égal à $\frac{1}{2}$, puisque $\rho=\frac{1}{\lambda}$, et l'égalité $(9)$, vérifiée pour une infinité de valeurs de $n$, prouve qu'il n'existe pas de nombre $\lambda$ tel que l'inégalité $(7)$ ait lieu quel que soit $n$.

On voit que la connaissance de l'exposant de convergence d'une suite est loin de renseigner d'une manière complete sur la rapidité de la croissance de son terme général $r_{n}$. Il est cependant un cas très important dans la pratique, bien qu'en apparence fort particulier, où l'on peut aboutir à des conclusions beaucoup plus pré- 
cises. C'est le cas où l'ordre d'infinitude de $r_{n}$, considéré comme fonction de n, est déterminé. Voici ce que nous entendrons par là; il est clair que l'on peut toujours poser

$$
r_{n}=n \lambda_{n},
$$

l'exposant $\lambda_{n}$ étant une certaine fonction de $n$.

Si nous marquons sur une droite l'ensemble des nombres dont l'abscisse est $\lambda_{n}$, cet ensemble admettra en général un ensemble dérivé, lequel, étant parfait, renfermera un élément $\lambda$. plus grand que tous les autres et un élément $\lambda^{\prime \prime}$ plus petit que tous les autres ( 1 ).

Les nombres $\lambda^{\prime}$ et $\lambda^{\prime \prime}$ sont ce que Cauchy appelait respectivement la plus grande et la plus petite des limites de la suite $\lambda_{n}\left({ }^{2}\right)$; Paul du Bois-Reymond leur a donné les noms de limite supérieure (ou inférieure) d'indétermination de la suite. Enfin M. Hadamard les nomme limite supérieure (ou inférieure) de $\lambda_{n}$ pour $n$ infini.

Nous supposons essentiellement ici que $\lambda^{\prime \prime}$ n'est pas nul $\left({ }^{3}\right)$, ce qui revient à admettre que les nombres $\lambda_{n}$ sont supérieurs à un nombre fixe ; dès lors, $\varepsilon$ étant un nombre positif arbitraire (4), on a, à partir d'une certaine valeur de $n$,

et, par suite,

$$
\lambda^{\prime \prime}-\varepsilon<\lambda_{n}<\lambda^{\prime}+\varepsilon,
$$

$$
n \lambda^{\lambda^{\prime}-\varepsilon}<r_{n}<n^{\lambda^{\prime}+\varepsilon}
$$

La fonction $r_{n}$ est ainsi comprise entre $n^{\lambda^{\prime \prime}-\varepsilon}$ et $n^{\lambda^{\prime}+\varepsilon}$, mais $l a$ considération de fonctions de la forme $n^{k}$ ( $k$ constante) ne

( $\left.{ }^{1}\right)$ On déduira aisément, du fait que les $r_{n}$ sont croissants, que l'ensemble dérivé est formé de tous les nombres compris entre $\lambda^{\prime \prime}$ et $\lambda^{\prime}$. Il est bon de remarquer que, si une infinité de $\lambda_{n}$ sont égaux, et que, par suite, une infinité de points coïncident en un seul, ce point doit être regardé comme faisant partie de l'ensemble dérivé, même s'il est isolé. Cette modification de détail aux définitions adoptẻes dans la théorie des ensembles de points parait devoir étre souvent avantageuse.

( $\left.{ }^{2}\right)$ Cours d'Analyse de l'École Polytechnique (OEuvres de Cauchy, II'série, i. III).

$\left({ }^{3}\right)$ On peut aussi étudier les ordres d'infinitude dans le cas où $\lambda_{n}$ tendrait vers zéro ou vers l'infini avec $n$; ee n'en est pas iri le lieu.

( $\left.{ }^{4}\right)$ Nous supposons aussi, pour plus de netteté, que $\lambda^{\prime}$ n'est pas infini; cette hypothèse n'est pas indispensable. 
permet pas de préciser davantage le mode de croissance de $r_{n}$. C'est ce que nous exprimerons en disant que, lorsque $\lambda^{\prime \prime}$ diffère de $\lambda^{\prime}$, l'ordre d'infinitude de $r_{n} n^{\prime}$ est pas déterminé; $\lambda^{\prime \prime}$ et $\lambda^{\prime}$ sont ses deux limites d'indétermination. Au contraire, si $\lambda^{\prime \prime}=\lambda^{\prime}=\lambda$, c'est-à-dire si $\lambda_{n}$ tend vers la limite $\lambda$, on a, quel que soit $\varepsilon$,

$$
n^{\lambda-\varepsilon}<r_{n}<n^{\lambda+\varepsilon},
$$

au moins à partir d'une valeur assez grande de $n$.

Nous dirons alors que l'ordre d'infinitude de $r_{n}$ est déterminé et est le même que celui de $n^{\lambda}$. Par exemple, si l'on a

$$
\begin{aligned}
& r_{n}=n^{\lambda}+n^{\lambda-1}, \\
& r_{n}=n^{\lambda} \log n, \\
& r_{n}=\frac{n^{\lambda}}{(\log n)^{q}},
\end{aligned}
$$

l'ordre d'infinitude de $r_{n}$ sera dit égal à celui de $n^{\lambda}$.

Il résulte immédiatement de ce qui précède que, lorsque l'ordre d'infinitude de $r_{n}$ est déterminé, il est égal à l'inverse de l'exposant de convergence de la suite

$$
r_{1}, r_{2}, \ldots, r_{n}, \ldots
$$

On a, en effet, l'égalité déjà écrite plus haut

$$
\lambda=\frac{\mathrm{I}}{\mathrm{\rho}} \text {. }
$$

Lorsque l'ordre d'infinitude de $r_{n}$ est indéterminé, sa limite inférieure d'indétermination $\lambda^{\prime \prime}$ est égale à $\frac{\mathrm{I}}{\rho}$, mais on ne peut rien dire sur $\lambda^{\prime}$ qui peut avoir toutes les valeurs supérieures $\grave{a} \lambda^{\prime \prime}$, jusque et $y$ compris $+\infty$.

On voit combien il est avantageux de savoir que l'ordre d'infinitude de $r_{n}$ est déterminé; lorsqu'il en est ainsi, la connaissance de l'exposant de convergence de la suite des $r_{n}$ permet d'obtenir immédiatement cet ordre, c'est-à-dire de préciser dans une assez large mesure la manière dont $r_{n}$ croît avec $n$. Ces remarques trouveront leur application dans la Note II. 


\section{CHAPITRE II.}

LES IDÉES DE LAGUERRE.

La notion de genre.

Laguerre a publié sur les fonctions entières, peu de temps après la découverte de Weierstrass, une série de Notes fort courtes, mais très substantielles et très suggestives (1). On sait quelle importance il altachait aux faits particuliers, mais bien précis, et combien il était peu porté à exposer systématiquement les idées générales qui, cependant, ne lui manquaient pas; il préférait les laisser deviner au lecteur, les dissimulant presque derrière les applications. Cette tendance apparaît très nettement dans ses travanx sur les fonctions entières; on se rend fort bien compte que sa pensée est allée plus loin qu'une lecture superficielle de ses publications ne le laisserait croire et qu'il a tout au moins entrevu les plus importants des résultats obtenus après lui.

D’ailleurs, l'étude des idées de Laguerre n'a pas seulement un intérêt historique; plusieurs de ses méthodes sont fort originales, et, dans les cas particuliers où elles s'appliquent, permettent d'aller bien plus loin que les méthodes plus générales que nous étudierons dans les Chapitres suivants. Il y aurait grand intérêt à rechercher si le champ d'application de ces méthodes ne peut pas être ètendu; c'est là un sujet de nature à tenter un jeune chercheur, car il peut être abordé sans connaissances très vastes, les méthodes de Laguerre étant, comme on va le voir, d'une nature élémentaire.

Le premier progrès que doit à Laguerre la théorie des fonctions

(1) Comptes rendus, Tomes XCIV, XCV, XCVIII. - OEuvres de Laguerre, Tome I, pages 167 et suiv. 
entières, c'est l'introduction de la notion de genre, notion qui a été l'origine de tous les travaux ultérieurs.

Soient $\mathrm{F}(z)$ une fonction entière $; a_{1}, a_{2}, \ldots, a_{n}, \ldots$ ses zéros; $r_{n}$ le module de $a_{n}$, les $r_{n}$ sont supposés croissants. Désignons par $k$ le plus petit nombre entier tel que la séric

$$
\sum \frac{1}{r_{n}^{k+1}}
$$

soit convergente. Nous avons vu que l'on a

$$
\mathrm{F}(z)=e^{Q(z)} \prod \mathrm{P}_{k}\left(\frac{z}{a_{n}}\right)
$$

$\mathrm{Q}(z)$ étant une fonction entière ou un polynome. Nous supposerons que $\mathrm{Q}(z)$ est un polynome et désignerons son degré par $q$. Laguerre appelle genre $p$ de la fonction $\mathrm{F}(z)$ le plus grand des deux nombres entiers $k$ et $q$. Dans le cas où il n'existe pas de nombre $k$ tel que la série ( 1 ) soit convergente el dans le cas où, ce nombre existant, $\mathrm{Q}(z)$ n'est pas un polynome, mais une fonction entière, on dit que le genre est infini. Nous étudierons presque exclusivement dans cet Ouvrage les fonetions de gerre fini.

L'un des deux nombres entiers qui nous ont servi à définir le genre est en relation simple avec l'exposant de convergence de la suite

$$
r_{1}, \quad r_{2}, \ldots, \quad r_{n}, \ldots
$$

En effet, ce nombre $k$ est défini par la condition que la série (1) est convergente, tandis que la série

$$
\sum \frac{1}{r_{n}^{\prime}}
$$

est divergente. On a donc, d'après la définition de l'exposant de convergence $\rho$ de la suite $(2)$

$$
k \leqq p \leqq k+\mathrm{r} .
$$

On voit que, lorsque le nombre $\rho$ n'est pas entier, $k$ est l'entier immédiatement inférieur à $\rho$; lorsque $\rho$ est entier, $k$ est égal à 
$\rho-1$ ou à $p$ suivant que la série

$$
\sum \frac{1}{r_{n}^{?}}
$$

est convergente ou divergente.

Nous conviendrons de dire que p est l'ordre réel(') de la fonction $\mathrm{F}(z)$. L'expression ordre réel est employée par opposition avec l'ordre apparent, qui sera défini plus loin. D'ailleurs nous verrons que, le plus souvent, ces deux ordres sont égaux, de sorte que, dans la plupart des cas, on pourra, sans ambiguïté possible, dire simplement ordre.

On voit que, lorsque l'ordre réel $p$ n'est pas entier, sa connaissance en apprend plus que celle du nombre $k$; au contraire, si $\rho$ cst entier, sa connaissance ne suffit pas pour déterminer $k$, qui pent être égal à $\rho$ ou à $\rho-1$; mais on doit évidemment regarder comme exceptionnel le cas où le nombre $\rho$ est un nombre entier, de sorte que l'on peut dire qu'en général l'étude de $\rho$ est préférable à celle de $k$. A plus forte raison est-elle préférable à celle du genre $p$ (lequel est égal soil à $k$, soit à $q$ ), si l'on a en vue seulement la connaissance de la distribution des zéros.

Il est cependant fort heureux que Laguerre n'ait pas introduit le nombre $p$; la théorie n'était pas'encore assez avancée pour que cette introduction pût être profitable et, d'autre part, il était tout à fait essentiel de faire jouer le même rôle aux deux nombres $k$ et $q$. Les propriétés les plus importantes de la fonction dépendent en effet, comme nous lc verrons, de la valeur du plus grand de ces deux nombres. C'est parce qu'elle suppose implicitement ce fait que la définition du genre a puissamment contribué au développement de la théorie. Il n'y avait pas grand effort à faire pour donner un nom au nombre $k$, ou au nombre $q$; la formule fonda-

( $\left.{ }^{1}\right)$ J'ai introduit pour la première fois eette expression dans mon Mémoire sur les zéros des fonetions entières (Acta mathematica, t. XX). Dans son Inaugural Dissertation (Göttingen, I898), un élève de M. Hilbert, M. von Schaper, s'est servi de l'expression exposant de convergence (Konvergenzexponent) pour désigner le nombre $p$. Nous l'avons adoptée, mais dans un sens légèrement différent : nous parlons de l'exposant de convergenee de la suite (2), mais non de l'exposant de convergence de la fonetion $\mathrm{F}(z)$. En résumé, nous appelons ordre réel d'une fonction entière l'exposant de convergence de la suite des modules de ses zéros (rangés toujours dans l'ordre croissant). 
mentale de Weierstrass suggérait d'ailleurs naturellement l'idée de considérer ces deux nombres. Le mérite principal de Laguerre, c'est.d'avoir vu qu'il y avait intérêt à porter l'attention sur le plus grand des deux, car ce point n'était nullement évident a priori ( $\left.{ }^{1}\right)$, et c'était cependant la condition nécessaire de tout progrès ultérieur.

Laguerre s'est particulièrement occupé des fonctions dont le genre est égal à zéro ou à un. Il n'est pas inutile de faire voir comment on peut rattacher les fonctions de genre fini aux fonctions de genre zéro; l'une des propositions que nous démontrerons à cette occasion nous sera d'ailleurs utile plus loin.

Soit $\mathrm{F}(z)$ une fonction de genre fini $p$; l'on a

$$
\mathbf{F}(z)=e^{Q(z)} \prod \mathrm{P}_{k}\left(\frac{\tilde{z}}{a_{n}}\right) ;
$$

le degré de $\mathrm{Q}(z)$ étant $q, p$ est égal au plus grand des deux nombres $q$ et $k$. Nous désignerons par $p$ l'exposant de convergence de la suite des modules des $a_{n}$

$$
r_{1}, r_{2}, \ldots, r_{n}, \ldots
$$

Nous savons que $\rho$ est au plus égal à $k+1$; d'ailleurs, dans le cas où $\rho=k+\mathrm{I}$, la série

$$
\sum \frac{1}{r_{i}^{?}}
$$

est convergente.

Cela posé, désignons par $m$ un entier supérieur à $p$ (par conséquent au moins égal à $p+1)$, par $\omega$ une racinc primitive de l'équation binome

$$
\omega^{m}=\mathrm{I},
$$

et formons le produit

$$
\mathrm{G}(z)=\mathrm{F}(z) \mathrm{F}(\omega z) \mathrm{F}\left(\omega^{2} z\right) \ldots \mathrm{F}\left(\omega^{m-1} z\right)
$$

( ${ }^{1}$ Par exemple, il aurait pu sembler étrange de ranger dans la mème catégoric (fonctions de genre un), les fonctions

$$
e^{z}, \quad e^{z} \prod_{1}^{\infty}\left(\mathrm{I}-\frac{\tilde{z}}{n^{2}}\right), \quad \prod_{1}^{\infty}\left(\mathrm{I}-\frac{\tilde{z}}{n}\right)^{\frac{z}{n}}, \quad \text { П }\left(\mathrm{I}-\frac{z}{\sqrt[3]{n^{2}}}\right) e^{\frac{3}{\sqrt[3]{n^{2}}}}
$$

dont les ordres réels sont respectivement $0, \frac{1}{2}, \frac{3}{2}$. 
On sait que si $\mathrm{R}(z)$ est un polynome de degré inférieur à $m$, l'on a

$$
\mathrm{R}(z)+\mathrm{R}(\omega z)+\ldots+\mathrm{R}\left(\omega^{m-1} z\right)=m \mathrm{R}(\mathrm{o}) .
$$

Or, le degré $q$ du polynome $\mathrm{Q}(z)$ est inférieur à $m$; d'autre part, les polynomes qui figurent comme exposant de $e$ dans les facteurs primaires de genre $k$ ont aussi un degré $k$ inférieur à $m$ et, de plus, s'annulent pour $z=0$. On a donc

$$
\mathrm{G}(z)=e^{m Q(0)} \prod\left(1-\frac{z^{m}}{a_{n}^{m}}\right) \cdot
$$

Posons, pour abréger,

il viendra

$$
\begin{aligned}
e^{m Q(0)} & =c, \\
z^{m} & =\mathrm{Z}, \\
a_{n}^{m} & =\mathbf{A}_{n} ;
\end{aligned}
$$

$$
\mathrm{G}(z)=c \prod\left(1-\frac{\mathrm{Z}}{\mathrm{A}_{n}}\right) .
$$

Si d'ailleurs on désigne par $\mathrm{R}_{n}$ le module de $\mathrm{A}_{n}$, l'exposant de convergence de la suite

$$
\mathrm{R}_{1}, \mathrm{R}_{2}, \quad \ldots, \mathrm{R}_{n}, \ldots
$$

est manifestement égal à $\frac{p}{m}$; il est inférieur à l'unité, sauf dans le cas particulier où, $p$ étant égal à $k+1$ et $q$ non supérieur à $k$, l'on a pris $m=p+\mathrm{I}=k+\mathrm{I}=\rho$. Dans ce cas, l'exposant de convergence de la suite des $\mathrm{R}_{n}$ est égal à l'unité, mais la série

$$
\sum_{\mathrm{R}_{n}}^{\frac{\mathrm{T}}{\mathrm{n}}}
$$

est convergente, ainsi qu'il résulte d'une remarque faite il y a un instant.

Dans tous les cas, on voit que $\mathrm{G}(\boldsymbol{z})$, considéré comme fonction de $\mathrm{Z}$, est une fonction de genre zéro et d'ordre $\frac{p}{m}$; nous ferons usage de ce résultat, obtenu, on le voit, par les considérations les plus élémentaires.

On peut énoncer une proposition bien plus complète, mais en utilisant un théorème que nous ne démontrerons que plus loin; ce théorème est le suivant : la somme de plusieurs fonctions de 
genre $p$ est une fonction de genre au plus égal ( 1$)$ à $p+\mathrm{I}$; il est d'ailleurs évident que le produil de plusieurs fonctions de genre $p$ est une fonction de genre égal à $p$. Dès lors, si, conservant les notations précédentes, mais désignant par $m$ un entier supérieur à $p+\mathrm{I}$, l'on pose

$$
\theta(\mathrm{Y})=[\mathrm{Y}-f(z)][\mathrm{Y}-f(\omega z)]\left[\mathrm{Y}-f\left(\omega^{2} z\right)\right] \ldots\left[\mathrm{Y}-f\left(\omega^{m-1} z\right)\right],
$$

les coefficients du polynome $\theta(Y)$ seront des fonctions entières dont le genre ne dépassera pas $p+\mathrm{I}$, c'est-à-dire sera inférieur à $m$. D'autre part, il est manifeste que $\theta(Y)$ reste invariable quand on change $z$ en $\omega z$; ces fonctions entières ne dépendent donc que de $z^{m}=\mathrm{Z}$, et l'on voit immédiatement que ce sont des fonctions de genre zéro $\left({ }^{2}\right)$ en $\mathrm{Z}$. La fonction $f(z)$ de genre $p$ est ainsi racine d'une équation de degré $m$,

$$
\mathrm{Y}^{m}+\mathrm{F}_{1}(\mathrm{Z}) \mathrm{Y}^{m-1}+\mathrm{F}_{2}(\mathrm{Z}) \mathrm{Y}^{m-2}+\ldots+\mathrm{F}_{m-1}(\mathrm{Z}) \mathrm{Y}+\mathrm{F}_{m}(\mathrm{Z})=\mathrm{o},
$$

dont les coefficients sont des fonctions entières de genre zéro en $\mathrm{Z}=z^{m}$.

Les fonctions de genre zéro eı de genre un.

On a vu que c'est la présence de facteurs exponentiels qui dislingue la décomposition des fonctions entières en facteurs primaires, de la décompositiou des polynomes en facteurs du premier degré. Il est dès lors naturel de penser que, parmi les fonctions entières, celles dont les propriétés se rapprocheront le plus de celles des polynomes seront les fonctions entières du genre zéro, qui peuvent être décomposées en un prodıit absolument convergent de facteurs du premier degré, sans aucun facteur exponentiel. Une induction plus hardie conduit même à supposer que les

(1) On prouve mème que c'est, en général, une fonction de genre $p$; il est infiniment probable qu'il en est toujours ainsi; mais on ne l'a pas encore démontré rigoureusement.

( $\left.{ }^{2}\right)$ D'ailleurs, en utilisant les propositions qui seront démontrées plus tard, on prouverait que leur ordre est au plus $\frac{\rho}{m}$; mais si $\rho=p+1$, et si l'on prenait $m=p+\mathbf{I}=p$, la difficulté serait de prouver que les fonctions obtenues, dont l'ordre est un, sont de genre zéro. C'est pour cela que nous avons supposé $m>p+r$. 
propriétés des fonctions entières s'éloigneront de plus en plus de celles des polynomes, à mesure que les facteurs exponentiels seront plus compliqués, et, dès lors, on pourra espérer étendre certaines propriétés des polynomes non seulement aux fonctions de genre zéro, mais encore à celles dont le genre est un nombre peu élevé.

Les idées que nous venons d'indiquer brièvement ont conduit Laguerre à plusieurs résultats sur les fonctions entières de genre zéro, de genre un et même de genre fini; ces résultats, à l'exposition desquels est consacré la fin de ce Chapitre, nous paraissent d'ailleurs surtout intéressants en tant qu'ils confirment l'analogie pressentie entre les fonctions considérées et les polynomes, et qu'en outre ils donnent des indications précieuses sur la distribution des zéros de certaines fonctions entières. Ils ouvrent ainsi la voie à une double série de recherches, comme on s'en rendra compte plus loin.

On désigne généralement sous le nom de théorème de Rolle la proposition d'après laquelle, entre deux racines réelles consécutives d'une équation, se trouve un nombre impair de racines de l'équation dérivée (et par suite au moins une). D'ailleurs, on peut démontrer cette proposition par des considérations de continuité, de sorte que, moyennant quelques hypothèses fort larges el inutiles à rappeler, le théorème de Rolle s'applique aussi bien aux équations transcendantes qu'aux équations algébriques.

Mais il n'en est pas de même de certaines de ses conséquences, dont l'importance en Algèbre est comparable à celle du théorème lui-même. On sait, par exemple, que, si l'on considère une équation algébrique dont toutes les racines sont réelles, l'équation dérivée a aussi toutes ses racines réelles; une seule de ses racines se trouve dans chaque interralle limité par deux racines consécutives de l'équation proposée; la démonstration de cette proposition, étant basée sur le fait que le nombre des racines de la dérivée est inférieur d'une unité au nombre des racines de l'équation, ne peut s'étendre aux équations transcendantes; il est d'ailleurs aisé de voir que la proposition ne peut subsister sans. restriction : par exemple, si l'on pose

$$
f(z)=(z+1) e^{z^{2}}
$$


on a

$$
f^{\prime}(z)=\left(2 z^{2}+2 z+1\right) e^{z^{2}}
$$

et l'on voit que l'équation

$$
f(z)=0
$$

n'admet qu'une racine, laquelle est réelle, tandis que l'équation

$$
f^{\prime}(z)=0
$$

admet deux racines imaginaires. Nous reviendrons dans le paragraphe suivant sur ce point, en étudiant les fonctions de genre fini; nous verrons avec quelles restrictions on peut leur étendre la proposition en question. Notre but actuel est de montrer qu'elle subsiste sans restrictions pour les fonctions de genre zéro et de genre $u n$; comme la fonction $f(z)$ qui vient d'être considérée est de genre deux et même l'une des fonctions les plus simples de genre deux, on voit que la limitation indiquée est bien dans la nature des choses et ne dépend pas seulement du procédé de démonstration.

Il est aisé de voir qu'une autre hypothèse restrictive est nécessaire pour les fonctions de genre un : on doit supposer la fonction réelle. Pour les fonctions de genre zéro, comme pour les polynomes, la réalité des racines entraîne la réalité de la fonction, à un facteur constant près, ici sans importance. Il n'en est pas de même pour les fonctions de genre un; on peut seulement affirmer qu'une telle fonction, si tous ses zéros sont réels, est de la forme

$$
\mathbf{F}(z)=e^{i(\alpha z+\beta)} \mathrm{G}(z),
$$

$\mathrm{G}(\approx)$ étant une fonction réelle, $\alpha$ et $\beta$ des constantes réelles; on a dès lors

$$
\mathrm{F}^{\prime}(z)=e^{i(\alpha z+\beta)}\left[\mathrm{G}^{\prime}(z)+i \alpha \mathrm{G}(z)\right]
$$

et l'on voit que toute racine réelle de l'équation

$$
F^{\prime}(z)=0
$$

doit satisfaire aux deux équations

$$
\begin{aligned}
& \mathrm{G}^{\prime}(z)=0, \\
& \mathrm{G}(z)=0,
\end{aligned}
$$

c'est dire que l'équation dérivée aura, en général, toutes ses racines imaginaires. 
La proposition que nous avons en vué s'énoncera donc comme il suit :

Étant donnée une fonction entière réelle $\mathrm{F}(z)$ de genre séro ou de genre un, si toutes ses racines sont réelles, on peut affirmer que la fonction dérivée $\mathrm{F}^{\prime}(z)$ a aussi toutes ses racines réelles et que, de plus, entre deux racines consécutives de $\mathrm{F}(z)$, se trouve une seule (') racine de $\mathrm{F}^{\prime}(z)$. Il en résulte que le genre de $\mathrm{F}^{\prime}(z)$ est égal à celui de $\mathrm{F}(z)$.

Soit, en effet,

$$
\mathrm{F}(z)=e^{k z \prod}\left(\mathrm{I}-\frac{z}{a_{n}}\right) \frac{z}{e^{\frac{z}{a_{n}}}}
$$

la fonction donnée; les nombres $a_{n}$ sont réels, ainsi que $k$; et la série

$$
\sum \frac{\mathrm{I}}{a_{n}^{2}}
$$

est convergente. Dans le cas où la série

$$
\sum \frac{1}{\left|a_{n}\right|}
$$

est convergente, si l'on a de plus

$$
k+\sum \frac{\mathrm{I}}{a_{n}}=0
$$

les facteurs exponentiels disparaissent et la fonction $\mathrm{F}(\boldsymbol{z})$ est de genre zéro. L'expression que nous avons écrite convient donc aux fonctions de genre zéro et de genre un, sous la seule hypothèse que la série (1) est convergente.

On a

$$
\frac{\mathrm{F}^{\prime}(z)}{\mathrm{F}(z)}=k+\sum\left(\frac{\mathrm{I}}{z-a_{n}}+\frac{\mathrm{I}}{a_{n}}\right)
$$

et l'on en conclut

$$
\frac{d}{d z}\left[\frac{\mathrm{F}^{\prime}(z)}{\mathrm{F}(z)}\right]=-\sum \frac{\mathrm{I}}{\left(z-a_{n}\right)^{2}} .
$$

La légitimité de ces opérations est assurée par la convergence

( $\left.{ }^{1}\right)$ Nous supposons, pour abréger, que toutes les racines de $F(z)$ sont simples; on sait que les racines multiples de $F(z)$ appartiennent aussi à $F^{\prime}(z)$; rien n'est d'ailleurs changé à nos conclusions. 
de la série (I). On voit que le rapport $\frac{F^{\prime}(z)}{F(z)}$ est constamment décroissant dans ses intervalles de continuité, car sa dérivée est essentiellement négative pour $\approx$ réel : il ne peat donc s'annuler qu'une fois dans chacun de ces intervalles. Ainsi se trouve démontrée la seconde partie de notre théorème.

Reste à faire voir que l'équation $\mathrm{F}^{\prime}(z)=0$ n'a pas de racines imaginaires; pour cela remplaçons (1), dans (2), $z$ par $x+i y$; nous obtenons

$$
\frac{\mathrm{F}^{\prime}(x+i y)}{\mathrm{F}(x+i y)}=k+\sum\left[\frac{x-a_{n}}{\left(x-a_{n}\right)^{2}+y^{2}}+\frac{1}{a_{n}}\right]-i \sum \frac{y}{\left(x-a_{n}\right)^{2}+y^{2}},
$$

les séries étant convergentes en même temps que la série (2). On voit que le coefficient de $-i$ se réduit au produit de $y$ par une somme essentiellement positive; il ne peut être nul que si l'on a

On ne peut donc avoir

$$
y=0 \text {. }
$$

$$
\mathrm{F}^{\prime}(x+i y)=0
$$

lorsque $y$ n'est pas nul ; notre proposition est donc complètement démontrée.

Reste à faire voir que le genre de $\mathrm{F}^{\prime}(z)$ est égal au genre de $\mathrm{F}(z)$; supposons d'abord que les $a_{n}$ soient tous positifs; désignons par $b_{0}$ la racine de la dérivée inférieure à $a_{1}$, si elle existe, et par $b_{1}, b_{2}, \ldots$ les autres racines de la dérivée; on a

$$
a_{1}<b_{1}<a_{2}<b_{2}<a_{3}<b_{3}<\ldots<a_{n}<b_{n}<a_{n+1}<\ldots
$$

On en conclut que, quel que soit le nombre positif $\rho$, la convergence de la série

entraîne celle de la série

$$
\sum \frac{1}{a_{i 2}^{P}}
$$

$$
\sum \frac{1}{b_{n}^{\circ}}
$$

et réciproquement. Les deux fonctions $F(z)$ et $F^{\prime}(z)$ ont donc non seulement. le même genre $\left({ }^{2}\right)$, mais encore le même ordre.

( ${ }^{1}$ ) Cette démonstration est due à Félix Chio (voir Hermite, Cours autographié, $4^{\mathrm{e}}$ édition, p. $\left.9^{2}\right)$.

$\left({ }^{2}\right)$ Pour que cette conclusion fút absolument rigoureuse, il serait nécessaire de prouver qu'il n'y a pas dans l'expression $\operatorname{de}^{\prime}(z)$ de facteur exponentiel $e^{G(z)}$

B. 
Plus généralement, si $\varphi(x)$ est une fonction positive décroissante, la convergence de la série

$$
\Sigma \varphi\left(a_{n}\right)
$$

entraîne celle de la série

$$
\Sigma \varphi\left(b_{n}\right)
$$

et réciproquement.

Il est aisé de s'affranchir de l'hypothèse que tous les $a_{n}$ sont positifs ;'il suffit de séparer, dans chacune des deux séries

$$
\begin{aligned}
& \sum \frac{1}{\left|a_{n}\right| \rho}, \\
& \sum \frac{1}{\left|b_{n}\right| \rho},
\end{aligned}
$$

les termes qui proviennent de racines positives de ceux qui proviennent de racines négatives; chacune de ces séries se trouve ainsi remplacée par deux autres, et il suffit de comparer deux à deux les quatre séries ainsi obtennes.

Le fait que le genre de $F^{\prime}(z)$ est égal à celui de $\mathbf{F}(z)$ a une conséquence très importante : nous pouvons appliquer le même théorème à $\mathbf{F}^{\prime}(z)$; ainsi toutes les équations dérivées ont leur's racines réelles, et, entre deux racines consécutives de la $n^{\text {ième }}$ se trouve une seule racine de la $(n+\mathbf{r})^{\text {ième }}$.

Laguerre a montré que l'équation (3) conduit à des résultats intéressants; en effectuant la dérivation indiquée dans le premier membre et retenant seulement que le second membre est essentiellement négatif pour $z$ réel, nous obtenons

$$
\mathrm{F}^{\prime \prime}(z) \mathbf{F}(z)-\left[\mathrm{F}^{\prime}(z)\right]^{2}<0 .
$$

Cette inégalité est une conséquence de la réalité des racines de $F(z)$; elle subsiste done si l'on remplace $F(z)$ par $F^{(n-1)}(z)$, c'est-à-dire que l'on a

$$
\mathrm{F}^{(n+1)}(z) \mathrm{F}^{(n-1)}(z)-\left[\mathrm{F}^{(n)}(z)\right]^{2}<0,
$$

quelle que soit la valeur réelle de $z$. En particulier, faisons $z=0$

de genre supérieur à un. C'est là un point que Laguerre a négligé d'étudier en détail; on peut compléter sa démonstration en suivant une marche analogue à celle que nous indiquons dans le dernier paragraphe de ce Chapitre, pour la proposition plus générale concernant les fonctions de genre fini. 
et posons

$$
\mathbf{F}(z)=\mathbf{A}_{0}+\mathbf{A}_{1} z-\ldots+\mathbf{A}_{n} z^{n}+\ldots,
$$

l'inégalité devient

$$
(n+\mathrm{I}) ! \mathbf{A}_{n+1}(n-\mathbf{I}) ! \mathbf{A}_{n-1}-\left[n ! \mathbf{A}_{n}\right]^{2}<0,
$$

c'est-à-dire

$$
(n+\mathrm{I}) \mathbf{A}_{n+1} \mathbf{A}_{n-1}-n \mathbf{A}_{n}^{2}<0 .
$$

Telle est l'inégalité à laquelle satisfont les coefficients de la fonction entière $F(z)$ sous les conditions que nous rappelons : la fonction $\mathrm{F}(z)$ est réelle, son genre ne dépasse pas l'unité et elle n'a pas de racines imaginaires.

En particulier, si l'on a

$$
\mathrm{A}_{n}=\mathrm{o},
$$

on en conclut

$$
\mathrm{A}_{n+1} \mathbf{A}_{n-1}<\mathrm{o}
$$

c'est-à-dire qu'une lacune (') ne peut se produire qu'entre deux coefficients de signes contraires. Sous une autre forme, on peut dire que, si dans le développement en série de la fonction réelle $\mathrm{F}(z)$ de genre inférieur à deux, il $y$ a une lacune entre deux coefficients de même signe, l'équation

$$
\mathrm{F}(z)=0
$$

admet nécessairement des racines imaginaires.

Laguerre donne comme exemple l'équation

$$
1+x \sin x=0
$$

dont le premier membre est de genre un, comme nous le verrons plus loin ( $\left.{ }^{2}\right)$.

(1) Il résulte manifestement de ce qui précède que deux coefficients consécutifs ne peuvent être nuls, car deux dérivées consécutives ne peuvent s'annuler pour $z=0$ [ sauf dans le cas où $F(z)$ admet $z=0$ comme racine multiple; alors les premiers coefficients sont nuls, mais notre remarque subsiste à partir du premier coefficient non nul].

( $\left.{ }^{2}\right)$ Laguerre affirme, sans démonstration, que ce genre est égal à l'unité; à moins d'une détermination directe des racines, qui constituerait ici un cercle vicieux, il nous semble que cette proposition ne peut ètre obtenuc que par lc théorème de M. Hadamard (ou un théorème analogue), et il n'est pas vraisemblable que Laguerre ait possédé un tel résultat (voir Chap. IV). 
L'inégalité (4) permet d'obtenir, dans le cas où les coefficients $\mathrm{A}_{n}$ sont tous positifs, une inégalité intéressante. Donnons, en effet, à $n$ successivement les valeurs $\mathbf{~}, 2,3, \ldots$, nous aurons

$$
\begin{aligned}
2 \mathbf{A}_{0} \quad \mathbf{A}_{2} & <\mathbf{A}_{1}^{2}, \\
3 \mathbf{A}_{1} \quad \mathbf{A}_{3} & <2 \mathbf{A}_{2}^{2}, \\
4 \mathbf{A}_{2} \quad \mathbf{A}_{4} & <3 \mathbf{A}_{3}^{2}, \\
5 \mathbf{A}_{3} \quad \mathbf{A}_{5} & <4 \mathbf{A}_{4}^{2}, \\
\ldots \ldots & \ldots \ldots \ldots, \\
n \mathbf{A}_{n} \quad \mathbf{A}_{n-2} & <(n-1) \mathbf{A}_{n-1}^{2}, \\
(n+1) \mathbf{A}_{n+1} \mathbf{A}_{n-1} & <n \mathbf{A}_{n}^{2},
\end{aligned}
$$

et en multipliant membre à membre, ce qui est permis, puisque les A sont tous positifs,

$$
(n+\mathrm{I}) \mathrm{A}_{0} \mathrm{~A}_{n+1}<\mathrm{A}_{1} \mathrm{~A}_{n},
$$

c'est-à-dire, en posant $\frac{A_{1}}{A_{0}}=c$,

$$
\mathbf{A}_{n+1}<\frac{c \mathbf{A}_{n}}{n+1}<\frac{c^{2} \mathbf{A}_{n+1}}{n(n+1)}<\ldots<\frac{c^{n+1} \Lambda_{0}}{(n+1) !} .
$$

Les coefficients de $\mathrm{F}(z)$ sont inférieurs aux coefficients correspondants de la série $\mathrm{A}_{0} e^{c x}$; tel est le résultat que nous voulions obtenir; il suppose que la fonction $\mathrm{F}(z)$, dont le genre est inférieur à deux, a ses racines réelles et ses coefficients tous positifs (les racines sont, dès lors, manifestement négatives).

Nous renverrons aux Notes déjà citées de Laguerre pour l'extension du théorème de Descartes aux fonctions entières.

\section{Les fonctions de genre fint.}

Nous allons, toujours d'après Laguerre, indiquer une application fort inléressante du théorème de Rolle aux fonctions de genre fini; le principe de la démonstration semble d'ailleurs pouvoir être utilisé dans d'autres recherches et mérite d'être connu. S'il a jusqu'ici passé à peu près inaperçu, c'est sans doute que la brièveté des explications données par Laguerre pouvait inspirer des doutes sérieux sur la rigueur de sa démonstration; j'espère avoir comblé 
les lacunes qui pouvaient y subsister; c'est ce que Laguerre aurait sans doute fait lui-même s'il avait publié son théorème ailleurs que dans les Comptes rendus; il paraît, dans tous les cas, bien probable qu'il possédait, explicitement ou implicitement, les éléments d'une démonstration complète et rigoureuse de son beau théorème.

Ce théorème peut s'énoncer comme il suit : Étant donnée une fonction entière $\mathrm{F}(z)$ de genre $p$ ayant un nombre fini $q$ de racines imaginaires : $1^{\circ}$ la fonction dérivée $\mathrm{F}^{\prime}(z)$ est de genre $p ; 2^{\circ}$ l'équation $\mathrm{F}^{\prime}(z)=0$ a, comme on sait d'après le théorème de. Rolle, une racine au moins dans l'intervalle de deux racines réelles consécutivès de l'équation $\mathrm{F}(z)=0$; on peut affirmer de plus que, en dehors de ces racines dont l'existence est décelée par le théorème de Rolle, il y a au plus $p+q$ autres racines, d'ailleurs réclles ou imaginaires.

Une fonction entière de genre fini $p$ est de la forme

$$
\mathrm{F}(z)=e^{Q(z)} \prod_{n=1}^{n=\infty}\left(\mathrm{I}-\frac{\tilde{z}}{a_{n}}\right) e^{\frac{z}{a_{n}}+\frac{z^{2}}{2 a_{n}^{z}}+\ldots+\frac{z p}{p a_{n}^{\nu}}}
$$

$\mathrm{Q}(z)$ étant un polynome de degré $p$ au plus. On a donc

$$
\mathrm{F}(z)=\lim _{m=\infty}\left[e^{Q(z)} \prod_{n=1}^{n=m}\left(\mathrm{I}-\frac{z}{a_{2}}\right) e^{\frac{z}{\bar{\pi}_{n}}+\ldots+\frac{z p}{p a_{n}^{p}}}\right],
$$

c'est-à-dire

$$
\mathrm{F}(\tilde{z})=\lim _{m=\infty} e^{Q_{m}(z)} \mathrm{P}_{m}(\tilde{z})
$$

en désignant par $\mathrm{P}_{m}(z)$ et $\mathrm{Q}_{m}(z)$ les deux polynomes

$$
\begin{aligned}
& \mathrm{P}_{m}(z)=\prod_{n=1}^{n=m}\left(1-\frac{z}{a_{n}}\right), \\
& Q_{m}(z)=\mathrm{Q}(z)+\sum_{n=1}^{n=m}\left(\frac{z}{a_{n}}+\ldots+\frac{z^{p}}{p a_{n}^{\nu}}\right),
\end{aligned}
$$

dont le dernier est au plus de degré $p$.

L'égalité (I) est fondamentale; remarquons d'ailleurs que 
$e^{Q(z)} \mathrm{P}_{m}(z)$ tend uniformément vers $\mathrm{F}(z)$ dans tout domaine fini; c'est une conséquence de la convergence uniforme du produit infini.

La démonstration de Laguerre repose essentiellement sur la différentiation de l'égalité (I), ce qui fournit l'égalité

$$
\mathbf{F}^{\prime}(z)=\lim _{m=\infty} \frac{d}{d z}\left[e^{Q_{m}(z)} \mathbf{P}_{m}(z)\right]
$$

nous allons montrer que cette opération est légitime, c'est-à-dire que l'égalité (I) a bien pour corrséquence l'égalité (2); on sait que, en général, de l'égalité

$$
\mathrm{F}(z)=\lim _{m=\infty} f_{m}(z)
$$

on n'a nullement le droit de conclure

$$
\mathrm{F}^{\prime}(z)=\lim _{m=\infty} f_{m}^{\prime}(z)
$$

Nous poserons

$$
\begin{gathered}
e^{Q_{m}(z)} \mathrm{P}_{m}(z)=\varphi_{m}(z) \\
\prod_{n=m+1}^{n=\infty}\left(\mathrm{I}-\frac{z}{a_{n}}\right) e^{\frac{z}{a_{n}}+\ldots+\frac{z^{p}}{a_{n}^{p}}}=\psi_{m}(z),
\end{gathered}
$$

de telle sorte que l'on a

$$
\mathrm{F}(z)=\varphi_{m}(z) \psi_{m}(z)
$$

on en conclut

$$
\mathrm{F}^{\prime}(z)=\varphi_{m}^{\prime}(z) \psi_{m}(z)+\varphi_{m}(z) \psi_{m}^{\prime}(z) .
$$

Nous voulons faire voir que, $z$ restant dans un domaine fine quelconque $(|z|<\mathrm{R}), \quad \varphi_{m}^{\prime}(z)$ tend uniformément vers $\mathrm{F}^{\prime}(z)$ lorsque $m$ augmente indéfiniment. Nous allons, dans ce but, évaluer la différence $\mathrm{F}^{\prime}(z)-\varphi_{m}^{\prime}(z)$. On a

$$
F^{\prime}(z)-\varphi_{m}^{\prime}(z)=\varphi_{m}^{\prime}(z)\left[\psi_{m}^{\prime}(z)-1\right]+\varphi_{m}(z) \psi_{m}^{\prime}(z) .
$$

Il est clair que, $|z|$ étant inférieur à $\mathrm{R}$, il existe un nombre $\mathbf{A}$ 
tel que l'on ait, quel que soit $m(1)$,

$$
\begin{aligned}
& \left|\varphi_{m}^{\prime}(z)\right|<A, \\
& \left|\vartheta_{m}(z)\right|<A .
\end{aligned}
$$

I'autre part, la convergence uniforme du produit infini permet, étant donné à l'avance le nombre $\varepsilon$, de trouver un nombre $\mu$, tel que pour $m>\mu$ l'on ait

$$
|\Psi m(z)-1|<\varepsilon .
$$

Dès lors, si nous prouvons que le nombre $\mu$ peut être choisi de telle manière que l'on ait aussi

$$
\left|\Psi_{m}^{\prime}(z)\right|<s,
$$

l'égalité (5) donnera, en tenant compte de $(6),(7),(8)$ et $(9)$,

$$
\left|F^{\prime}(z)-p_{m}^{\prime}(z)\right|<2 \varepsilon A,
$$

sous les conditions

$$
\left\{\begin{array}{l}
|z|<\mathbf{R} \\
m>\mu
\end{array}\right.
$$

le nombre $\mu$ pouvant d'ailleurs être déterminé quels que soient les nombres donnés d'avance $R$ et $\varepsilon$. C'est bien le résultat que nous désirons obtenir.

Tout revient donc à prouver la possibilité de déterminer $u$, lorsqu'on donne $\mathrm{R}$ el $\varepsilon$, de manière à vérificr l'inégalité (9) sous les conditions (10). Pour cela, prenons la dérivée logarithmique de l'égalité (4), qui définit $\bigcup_{m}(z)$; nous obtenons

$$
\frac{\Psi_{m}^{\prime}(z)}{\Psi_{m}(z)}=\sum_{n=m+1}^{n=\infty}\left(\frac{1}{z-a_{n}}+\frac{1}{a_{n}}+\frac{z}{a_{n}^{2}}+\ldots+\frac{z^{p-1}}{a_{n}^{p}}\right) .
$$

( $\left.{ }^{1}\right)$ Pour démontrer l'inégalité (6), on peut remarquer que si l'on considère un contour simple $\Gamma$ enveloppant le cercle de rayon $R$ et sur lequel il $n$ 'y ait pas de zéro de $\mathrm{F}(z)$, il existe un nombre positif $B$ tel que l'on ait sur ce contour $\left|\varphi_{m}\right|>\mathrm{B}$ et un nombre $\mathrm{C}$ tel que l'on ait aussi sur tout le contour $\left|\frac{\rho_{m}^{\prime}}{\varphi_{m}}\right|<\mathrm{C}$; dès lors, si l'on pose $\mathbf{A}=\mathrm{BC}$, l'inégalité ( 6 ) est vérifiée sur tout $\Gamma$ et par suite en tout point intérieur. 
Prenons $m$ assez grand pour que l'on ait

nous pourrons écrire

$$
\left|a_{m+1}\right|>\mathrm{R}
$$

$$
\frac{\psi_{m}^{\prime}(z)}{\psi_{m}(z)}=-z^{p} \sum_{n=m+1}^{n=\infty} \frac{1}{a_{n}^{p+1}}-z^{p+1} \sum_{n=m+1}^{n=\infty} \frac{1}{a_{n}^{p+2}}-\ldots
$$

Or, en désignant par $r_{n}$ le module de $a_{n}$, on a visiblement, $r_{n}$ croissant avec $n$,

$$
\begin{aligned}
& \left|\sum_{n=m+1}^{n=\infty} \frac{1}{a_{n}^{p+1}}\right|<\sum_{n=m+1}^{n=\infty} \frac{1}{r_{n}^{p+1}}, \\
& \left|\sum_{n=m+1}^{n=\infty} \frac{1}{a_{n}^{p+k}}\right|<\frac{1}{r_{m+1}^{k}} \sum_{n=m+1}^{n=\infty} \frac{1}{r_{n}^{p+1}} .
\end{aligned}
$$

Dès lors, si nous posons

$$
\sum_{n=m+1}^{n=\infty} \frac{1}{r_{n}^{p+1}}=n_{m}
$$

il vient, puisque le module de $z$ est inférieur à $R$,

$$
\left|\frac{\psi_{m}^{\prime}(z)}{\psi_{m}(z)}\right|<\mathrm{R}^{p} r_{i m}\left(\mathrm{I}+\frac{\mathrm{R}}{r_{m+1}}+\frac{\mathrm{R}^{2}}{r_{m+1}^{2}}+\ldots\right),
$$

c'est-à-dire

$$
\left|\psi_{m}^{\prime}(z)\right|<\left|\psi_{m}(z)\right| \frac{\mathrm{R}^{p}}{1-\frac{\mathrm{R}}{r_{m+1}}} \eta_{m} .
$$

Cette inégalité établit notre proposition, car $\left|\psi_{m}(z)\right|$ tend uniformément vers un, le facteur $\frac{\mathrm{R}^{p}}{1-\frac{k}{r_{m+1}}}$ est fini, et la série

$$
\sum \frac{1}{r_{n}^{p+1}}
$$

étant convergente, on peut choisir $\mu$ de manière que $\eta_{m}$ soit inférieur à un nombre donné d'avance pour toutes lcs valeurs de $m$ supérieures à $\mu$. 
On démontrerait de même que $\varphi_{i m}^{\prime \prime}(z)$ tend uniformément vèrs $F^{\prime \prime}(z)$ ( $z$ restant dans un domaine fini quelconque donné d'avance). On écrirait d'abord l'égalité

$$
\mathrm{F}^{\prime \prime}(z)=\varphi_{m}^{\prime \prime}(z) \psi_{m}(z)+2 \varphi_{m}^{\prime}(z) \psi_{m}^{\prime}(z)+\varphi_{m}(z) \psi_{m}^{\prime \prime}(z)
$$

et il suffirait de démontrer que $\varphi_{m}^{\prime \prime}(z)$ tend uniformément vers zéro; on y arrive aisément en prenant la dérivée des deux membres de (I $)$; on obtient

$$
\frac{\psi_{m}^{\prime \prime}(z)}{\psi_{m}(z)}=\frac{\psi_{m}^{\prime 2}(z)}{\psi_{m(m}^{2}(z)}+\sum_{n=m+1}^{\infty}\left[\frac{-1}{\left(z-a_{n}\right)^{2}}+\frac{1}{a_{n}^{2}}+\frac{2 z}{a_{n}^{3}}+\ldots+\frac{(p-1) z^{p-2}}{a_{n}^{\prime \prime}}\right]
$$

et l'on montre par le procédé déjà employé que la série peut être rendue aussi petite que l'on veut, en prenant $m$ assez grand.

Nous pouvons donc énoncer le résultat suivant:

Étant donnée la fonction de genre $p$ :

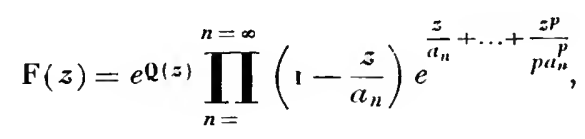

on obtient, en prenant seulement $m$ facteurs dans le produit infinı

$$
\mathrm{F}(z)=\lim _{m=\infty} e^{\mathbf{Q}_{m}(z)} \mathrm{P}_{m}(z)=\lim _{m=\infty} \varphi_{m}(z)
$$

et l'on a

$$
\begin{aligned}
& \mathbf{F}^{\prime}(z)=\lim _{m=\infty} \frac{d}{d z}\left[e^{Q_{m}(z)} P_{m}(z)\right]=\lim _{m=\infty} \varphi_{m}^{\prime}(z), \\
& \mathbf{F}^{\prime \prime}(z)=\lim _{m=\infty} \frac{d^{z}}{d z^{2}}\left[e^{Q_{m}(z)} P_{m}(z)\right]=\lim _{m=\infty} \wp_{m}^{\prime \prime}(z)
\end{aligned}
$$

D'ailleurs, lorsque $z$ est dans un donaine fini quelconque donné d'avance, $\varphi_{m}, \vartheta_{m}^{\prime}, \varphi_{m}^{\prime \prime}$ tendent uniformément vers leurs limites.

Ces résultats sont établis indépendamment de toute hypothèse sur la réalité des zéros $a_{n}$; nous allons maintenant supposer que, sauf un nombre limité, ces zéros sont réels et distincts (la dernière hypothèse n'est pas nécessaire; rien d'essentiel ne serait changé s'il y avait des zéros multiples, mais les raisonnements seraient allongés sans aucun profit). Pour préciser nous supposerons que, parmi les $a_{n}, q$ seulement sont imaginaires; de plus nous 
supposons que la fonction $\mathrm{F}(z)$ est réelle ( $q$ est dès lors un nombre pair). Le polynome $\mathrm{P}_{m}(z)$, dont les zéros sont $a_{1}, a_{2}, \ldots$, $a_{m}$, a donc au moins $m-q$ zéros réels; l'équation

$$
\varphi_{m}(z)=0
$$

ayant $m-q$ racines réelles, l'équation dérivée

$$
\varphi_{m}^{\prime}(z)=0
$$

a au moins $m-q-1$ racines réelles, lesquelles sont comprises dans les intervalles formés par les $m-q$ premiers zéros réels de $F(z)$. On a d'ailleurs

$$
\begin{gathered}
\varphi_{m}(z)=e^{Q_{m}(z)} \mathrm{P}_{m}(z), \\
\varphi_{m}^{\prime}(z)=e^{Q_{m}(z)}\left[\mathrm{P}_{m}^{\prime}(z)+\mathrm{Q}_{m}^{\prime}(z) \mathrm{P}_{m}(z)\right] .
\end{gathered}
$$

Le degré de $\mathrm{Q}_{m}(z)$ étant au plus égal à $p$, on voit que $\varphi_{m}^{\prime}(z)$ est le produit d'un facteur exponentiel par un polynome de degré au plus égal à $m+p-1$; donc $\stackrel{o}{i}_{m}^{\prime}(z)$ a au plus $m+p-$ I zéros et comme $m-q-\mathrm{r}$ au moins sont réels, il $y$ en a au plus :

$$
m+p-1-(m-q-1)=p+q
$$

qui sont imaginaires ( $\left.{ }^{1}\right)$.

Or $\varphi_{m}^{\prime}(z)$ a pour limite $\mathrm{F}^{\prime}(z)$; on est ainsi porté à croire que $\mathbf{F}^{\prime}(z)$ admet au plus $p+q$ zéros imaginaires; il est aisé de vérifier cette induction par un raisonnement rigoureux.

Considérons un contour fermé quelconque $C$ sur lequel ne se trouve aucun zéro de $F^{\prime}(z)$; nous supposerons, pour fixer les idées, que $\mathrm{C}$ est un contour simple, par exemple un cercle de rayon $R$.

Il est clair qu'il existe un nombre $\boldsymbol{H}$ tel que l'on ait, sur tout le contour C,

$$
\left|\mathrm{F}^{\prime}(z)\right|>\mathrm{II}
$$

D'autre part, $\vartheta_{m}^{\prime}(z)$ tendant uniformément vers $\mathrm{F}^{\prime}(z)$, on peut déterminer un nombre $\mu$ tel que, pour $m>\mu$, l'on ait, quel que soit $\approx$ sur C,

$$
\left|\mathrm{F}^{\prime}(z)-\varphi_{m}^{\prime}(z)\right|<\frac{\mathrm{H}}{2}
$$

( $\left.{ }^{1}\right)$ On peut remarquer que, lorsque $p$ est impair, $p+q$ est impair et, par suite, $\vartheta_{m}(z)$ étant réel a au plus $p+q-$ I zéros imaginaires. 
et dès lors on a, quel que soit $m>\mu$ et quel que soit $z$ sur $C$,

$$
\left|\varphi_{m}^{\prime}(z)\right|>\frac{H}{2} \text {. }
$$

Cela posé, considérons l'intégrale

$$
\mathrm{J}_{m}=\frac{\mathrm{I}}{2 i \pi} \int_{\mathbf{C}} \frac{\varphi_{m}^{\prime \prime}(z) d z}{\bigcup_{m}^{\prime}(z)},
$$

et faisons croître indéfiniment $m$ à partir de la valeur $\mu$. Il résulte manifestement de ce qui précède que l'on a

$$
\lim _{m=\infty} \mathrm{J}_{m}=\mathrm{J}=\frac{\mathrm{I}}{2 i \pi} \int_{\mathrm{C}} \frac{\mathrm{F}^{\prime \prime}(z) d z}{\mathrm{~F}^{\prime}(z)} .
$$

Mais on sait que $\mathbf{J}_{m}$ est un nombre entier, égal au nombre des zéros de $\varphi_{m}^{\prime}(z)$ compris à l'intérieur de C. Donc, ce nombre est constant à partir d'une certaine valeur de $m$ (laquelle peut dépendre du contour C) et égal au nombre des zéros de $\mathrm{F}^{\prime}(\xi)$ compris à l'intérieur du même contour.

Cela posé, prenons un contour $\mathrm{C}$ renfermant à son intérieur les $h$ zéros réels $a_{n}$ de $\mathrm{F}(z)$ dont le module est le plus petit; supposons, de plus, pour plus de netteté, que le contour C rencontre la partie positive et la partie négative de l'axe réel en deux points ( 1 ) qui soient des zéros de $F(z)$ (non comptés parmi les $h$ intérieurs). Le contour $\mathrm{C}$ comprend ainsi à son intérieur $h+\mathrm{I}$ des intervalles I que séparent sur l'axe réel les zéros de $F(z)$ et, par suite, $h+\mathrm{I}$ zéros réels de $\mathrm{F}^{\prime}(z)$, puisque chacun de ces intervalles en renferme au moins un.

D'autre part, dès que $m-q$ dépasse $h+2$, parmi les $m-q-1$ zéros réels de $\vartheta_{m}(z)$, compris dans les intervalles $I$, $h+1$ seulement sont intérieurs à $\mathrm{C}$; les autres, au nombre de

$$
m-q-\mathrm{r}-(h+1)=m-q-h-2,
$$

sont extérieurs à $\mathrm{C}$; nous ne savons d'ailleurs rien sur les $p+q$

( $\left.{ }^{1}\right)$ Une légère modification devrait ètre introduite dans le cas où les $a_{n}$ réels n'augmenteraient pas indéfiniment par valeurs tantôt positives, tantôt négatives, mais par valeurs toutes de mème signe 
autres zéros de $\varphi_{m}(z)$ (qui peuvent ètre réels ou imaginaires), mais le nombre total des zéros de $\varphi_{m}(z)$ étant $m+p-\mathbf{I}$, il y en a au plus

$$
m+p-1-(m-q-h-2)=p+q+h+1
$$

à l'intérieur de $\mathrm{C}$. Ce nombre est indépendant de $m$; donc $\mathrm{F}^{\prime}(z)$ a au plus $p+q+h+\mathrm{x}$ zéros à l'intérieur de C. Or, nous connaissons déjà les $h+1$ zéros compris dans les intervalles I; donc il y a au plus $p+q$ zéros de $\mathrm{F}^{\prime}(z)$ extérieurs à ces intervalles et intérieur's à C. Mais rien n'empêche de faire croître indéfiniment le nombre $h$ et avec lui le contour $C$; le même résultat subsiste toujours, et nous arrivons à l'important résultat de Laguerre. En dehors des zéros compris dans les intervalles I (un dans chaque intervalle) et dont l'existence est une conséquence nécessaire du théorème de Rolle, la fonction $\mathrm{F}^{\prime}(z)$ a au plus $p+q$ zéros sur la position desquels on ne sait rien. lin particulier, si $q=0$, e'est-à-dire si l'équation

$$
\mathrm{F}(z)=0
$$

a toutes ses racines réelles, l'équation

$$
\mathrm{F}^{\prime}(z)=0
$$

a au plus p racines imaginaires. Ce nombre maximum se réduit d'ailleurs évidemment à $p-\mathrm{I}$ lorsque $p$ est impair, de sorte que nous retrouvons, comme cas particulier, l'un des résultats du paragraphe précédent.

Il reste à prouver que la dérivée $F^{\prime}(z)$ cst bien de genre $p$. D'abord, ou démontrera comme précédemment que si l'on désigne par $b_{n}$ les zéros de $\mathrm{F}^{\prime}(z)$, la série

$$
\sum \frac{1}{\left|b_{n}\right| p}
$$

est convergente ou divergente en même temps que la série

$$
\sum \frac{1}{\left|a_{n}\right| p}
$$

Cela résulte de ce que les $p+q$ zéros, sur la position desquels on ne sait rien, sont sans influence sur la convergence ou la diver- 
gence; les autres sont situés dans les intervalles $I$, à raison d'un et d'un seul dans chaque intervalle.

Considérons maintenant un point $b_{n}$ et entourons-le d'un cercle $\gamma_{n}$ assez petit pour ne renfermer à son intérieur aucun autre zéro de $F^{\prime}(z)$; il résulte de ce qui précède que, à partir d'une certaine valeur de $m$, ce cercle contiendra un zéro et un seul de $\varphi_{m}^{\prime}(z)$; nous dirons que ce zéro de $\varphi_{m}^{\prime}(z)$ est celui quı tend ver's $b_{n}$; il est clair, en effet, que sa distance à $b_{n}$ tend vers zéro lorsque $m$ croìt indéfiniment, puisque le cercle $\gamma_{n}$ peut être pris aussi petit que l'on veut.

Remarquons maintenant que, étant donné un cercle de rayon $\mathbf{R}$ aussi grand que l'on veut, mais fixe, on peut prendre $\mu$ assez grand pour que, lorsque $m$ dépasse $\mu$, chaque zéro de $\varphi_{m}^{\prime}(z)$ intérieur à ce cercle tende vers un zéro déterminé de $F^{\prime}(z)$. C'est une conséquencè du fait que $\mu$ peut être pris assez grand pour que les zéros de $\varphi_{m}^{\prime}(z)$ soient en même nombre que ceux de $\mathrm{F}^{\prime}(z)$; dès lors, ceux-ci étant en nombre limité, on peut choisir les cercles $\gamma_{n}$ et déterminer ensuite $\mu$ en prenant le plus grand des nombres $m$ qui correspondent à chacun de ces cercles.

Ces préliminaires étant établis, désignons par

$$
\beta_{1}^{(m)}, \beta_{2}^{(m)}, \ldots, \beta_{n}^{(m)}, \ldots, \beta_{m+p-1}^{(m)}
$$

les zéros de $\varphi_{m}^{\prime}(z)$ et supposons que $\beta_{n}^{(m)}$ ait pour limite $b_{n}$ lorsque $m$ angmentc indéfiniment ( 1 ). Dès lors, considérons les deux produits

$$
\begin{aligned}
& \Gamma_{m}(z)=\prod_{n=1}^{n=m+p-1}\left(1-\frac{z}{\beta_{, l}^{(m)}}\right) e^{\frac{z}{\beta_{n}^{m}}+\frac{1}{2}\left(\frac{z}{\beta_{n}^{(m)}}\right)^{2}+\ldots+\frac{1}{p}\left(\frac{z}{\beta_{n}^{(m)}}\right)^{p},} \\
& \mathrm{G}(z)=\prod_{n=1}^{n=\infty}\left(1-\frac{z}{b_{n}}\right) e^{\frac{z}{b_{n}}+\frac{1}{2}\left(\frac{z}{b_{n}}\right)^{2}+\ldots+\frac{1}{p}\left(\frac{z}{b_{n}}\right)^{p}} .
\end{aligned}
$$

Ce dernier est convergent à canse de la remarque faite tout à l'heure et représente par suite une fonction entière.

( ${ }^{1}$ Lorsque l'on donne $n$, on peut déterminer, à partir d'une certaine valeur de $m$, quelle est celui des zéros de $\varphi_{m}^{\prime}(z)$ qui tend vers $b_{n}$; on le désigne par $\beta_{h}^{(m)}$. Pour les valeurs inférieures de $m$, il subsiste un certain arbitraire dans la notation; mais on verra que cela n'a pas d'importance. L'essentiel est que $\beta_{n}^{(m)}$ ait un sens précis, lorsque $n$ est donné, pourvu que $m$ soit assez grand. 
Nous allons prouver que lorsque m augmente indéfiniment, $\Gamma_{m}(z)$ tend uniformément vers $\mathrm{G}(\xi)$ à l'intérieur de tout domaine fini. En d'autres termes, quels que soient les nombres donnés d'avance $\mathrm{R}$ et $\varepsilon$, on peut déterminer $\mu$ de manière que l'on ait

$$
\left|\Gamma_{m}(z)-\mathrm{G}(z)\right|<\varepsilon,
$$

sous les seules conditions

$$
m>\mu, \quad|z|<\mathbf{R} .
$$

Nous omettrons la démonstration de cette proposition, qui est un peu longue, mais qui ne présente aucune difficulté et que le lecteur reconstituera aisément. Il importe seulement d'observer qu'il ne suffit pas de savoir que $\beta_{n}^{(m)}$ tend vers $b_{n}$; il faut encore s'appuyer sur deux remarques que nous avons eu soin de faire: $1^{\circ}$ sauf un nombre limité, les $b_{n}^{(m)}$ sont dans les intervalles $1 ; 2^{\circ}$ un contour $\mathrm{C}$ et un nombre $n$ étant donnés, on peut déterminer $m$ de manière que chaque $\beta_{n}^{(m)}$ intérieur à $C$ soil à une distance de $b_{n}$ inférieure à $\eta$.

Reprenons maintenant l'égalité

$$
\mathrm{F}^{\prime}(z)=\lim _{m=\infty} \varphi_{m}^{\prime}(\tilde{z})=\lim _{m=\infty} e^{\mathbf{Q}_{m}(z)} \mathrm{P}_{m}(z)
$$

Remarquons que $\Gamma_{m}(z)$ ayant les mêmes zéros que $\varphi_{m}^{\prime}(z)$, l'on a

$$
\mathrm{\Gamma}_{m}(z)=e^{\mathbf{R}_{m}(z)} \mathrm{P}_{m}(z)
$$

d'ailleurs

$$
\mathrm{G}(z)=\lim _{m=\infty} \Gamma_{m}(z)
$$

On a donc pour toute valeur de $z$

$$
\frac{\mathrm{F}^{\prime}(z)}{\mathrm{G}(\tilde{z})}=\lim _{m=\infty} \frac{\varphi_{m}^{\prime}(z)}{\Gamma_{m}(z)}=\lim _{m=\infty} e^{Q_{m}(z)-\mathrm{R}_{m}(z)}
$$

car la seule difficulté pourrait résulter des zéros de $\mathrm{G}(z)$ et de $\mathrm{I}_{m}(z)$, mais elle disparaît, puisque ces zéros appartiennent respeclivement à $\mathrm{F}^{\prime}(z)$ et à $\varphi_{m}^{\prime}(z)$.

IIais $\mathrm{Q}_{m}(z)-\mathrm{R}_{m}(z)$ est un polynome de degré p au plus; nous voyons que ce polynome tend vers une limite lorsque $m$ augmente indéfiniment, c'est-à- dire qu'il existe une fonction bien 
déterminée $\mathrm{S}(z)$ telle que l'on ait, quel que soit $z$,

$$
\lim _{m=\infty}\left[\mathrm{Q}_{m}(z)-\mathrm{R}_{m}(z)\right]=\mathrm{S}(z)
$$

Il est clair que $\mathrm{S}(z)$ ne peut être qu'un polyriome de degré au plus égal à $p$ et l'on a

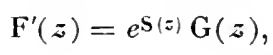

ce qui prouve bien que le genre de $F^{\prime}(z)$ est égal à $p$. C'est ce que nous voulions établir. Nous démontrerons plus loin un résultat bien plus général, puisque nous ne supposerons rien sur la réalité des racines de $\mathrm{F}(z)$, mais un peu moins précis, car, dans certains cas, il restera douteux si le genre de $\mathbf{F}^{\prime}(z)$ est $p$ ou $p+\mathbf{I}$.

Il n'est pas invraisemblable qu'il y ait moins de difficultés à lever ce doute par des méthodes de nature algébrique, analogues à celle que nous venons de développer d'après Laguerre, que par les méthodes à caractère nettement transcendant auxquelles est consacré la fin de cet Ouvrage et dont nous trouverons l'origine dans un important Mémoire de M. Poincaré. Peu de travaux ont été faits dans cette direction; signalons cependant des recherches intéressantes de MM. Cesaro ( $)$, Vivanti $\left({ }^{2}\right)$, Bassi $\left({ }^{3}\right)$, se rapportant surtout aux fonctions de genre quelconque privées de facteur exponentiel. Bien que cette dernière hypothèse soit assez artificielle et ne soit guère justifiée que par le désir d'obtenir des résultats simples, les méthodes suivies par ces divers géomètres sont peut-être susceptibles d'extension et il y avait lieu de les mentionner.

( $\left.{ }^{1}\right)$ Giornale di Battaglini, t. XXII (188') et Comptes rendus, t. XCIX.

(2) Ibid., t. XXII et XXIII (188'-1885).

$\left({ }^{3}\right)$ Rendiconti del R. Ist. Lomb., série II, vol. XXVI (1895). 


\section{CHAPITRE III.}

LES INÉGALITÉS DE M. POIXCARÉ.

Dans son Mémoire sur les fonctions entières ('), M. Poincaré a mis en évidence deux faits de la plus grande importance : il a indiqué une relation, d'une part, entre l'ordre de grandeur d'une fonclion entière el son genre supposé fini, et, d'autre part, entre l'ordre de grandeur de la fonction et l'ordre de grandeur de ses coefficients. Le premier paragraphe de ce Chapitre est consacré à l'exposition des résultats mêmes de M. Poincaré, les deux suivants, à des développements qui se rattachent respectivement aux deux faits qui viennent d'ètre signalés.

\section{Le Mémoire de M. Poincaré.}

Considérons d'abord une fonction de genre zéro

$$
\mathrm{F}(z)=\left(1-\frac{z}{a_{1}}\right)\left(1-\frac{z}{a_{2}}\right) \cdots\left(1-\frac{z}{a_{n}}\right) \cdots,
$$

la série

$$
\sum\left|\frac{1}{a_{n}}\right|
$$

est supposée convergente. Nous allons prouver que, $\alpha$ étant un nombre réel et positif quelconque, si l'on désigne par $r$ le module de $z$, on a

$$
\lim _{z=\infty} e^{-\alpha r} \mathbf{F}(z)=0 .
$$

En d'autres termes, a élant choisi, comme il a été dit, si l'on se donne un nombre positif arbitraire $\varepsilon$, on pourra trouver un nombre $c$

(1) Bull. de la Soc. math. de France, is83. 
tel que l'inégalité

entraîne

$$
r>c
$$

$$
\left|e^{-\alpha r} \mathrm{~F}(z)\right|<\varepsilon .
$$

Pour le prouver, remarquons que, la série (I) étant convergente, on peut trouver des nombres positifs (non nuls)

$$
x_{1}, \quad x_{2}, \ldots, x_{n}, \ldots,
$$

tels que l'on ait

$$
x=x_{1}+x_{2}+\ldots+x_{n}+\ldots
$$

et que de plus, à partir d'une certaine valeur de $n$, l'inégalité

$$
\left|\frac{1}{a_{n}}\right|<\alpha_{n}
$$

soit vérifiée ('). Supposons-la vérifiée pour $n>m$; le nombre $n \iota$ est fixe, c'est à-dire ne dépend pas de $z$. Nous voulons étudier le produit $e^{-x r} \mathbf{F}(z)$; nous écrirons

$$
e^{-\varkappa r} \mathrm{~F}(z)=e^{-x_{1} r}\left(1-\frac{z}{a_{1}}\right) e^{-\gamma_{2} r}\left(1-\frac{z}{a_{2}}\right) \ldots e^{-x_{n} r}\left(1-\frac{z}{a_{n}}\right) \cdots
$$

et nous poserons

$$
\begin{aligned}
& \mathrm{P}_{m}=\prod_{\mathrm{I}}^{m}\left[e^{-x_{n} r}\left(\mathrm{I}-\frac{z}{a_{n}}\right)\right], \\
& \mathrm{R}_{m}=\prod_{m+1}^{\infty}\left[e^{-x_{n} r}\left(\mathrm{I}-\frac{z}{a_{n}}\right)\right] .
\end{aligned}
$$

de telle sorte que l'on a

$$
e^{-\chi r} \mathbf{F}(z)=\mathbf{P}_{m} \mathbf{R}_{m}
$$

(') En effet, la série (I) étant convergente, il existe un nombre $m$ tel que lon ait

$$
2 \sum_{m+1}^{\infty}\left|\frac{x}{a_{n}}\right|=\beta<\alpha .
$$

On prendra, pour $n \leqq m, x_{n}=\frac{\alpha-\beta}{m}$ et pour $n>m, x_{n}=2\left|\frac{1}{a_{n}}\right|$.

B. 
D'après la forme même du produit $\mathrm{P}_{m}$ il est clair que l'on peut prendre $r$ assez grand pour vérifier l'inégalité (')

$$
\left|\mathrm{P}_{m}\right|<\varepsilon \text {. }
$$

Nous allons montrer, d'autre part, que l'on a, pour toute valeur de $z$,

$$
\left|\mathrm{R}_{m}\right| \leqq I
$$

il en résultera bien

$$
\left|\mathrm{P}_{m} \mathrm{R}_{m}\right|=\left|e^{-x r} \mathrm{~F}(z)\right|<\varepsilon .
$$

Pour démontrer l'inégalité (3) il suffit de faire voir que le module de chaque facteur de $R_{m}$ est au plus égal à l'unité. Or on a

Done

$$
\left|\mathrm{t}-\frac{z}{a_{n}}\right| \leqq \mathbf{1}+\left|\frac{z}{a_{n}}\right|=e^{\left|\frac{z}{a_{n}}\right|} .
$$

$$
\left|e^{-\alpha_{n} r}\left(1-\frac{z}{a_{n}}\right)\right| \leqq e^{\left(\left|\frac{1}{a_{n}}\right|-\alpha_{n}\right) r} \leqq 1,
$$

puisque $\left|\frac{1}{a_{n}}\right|-\alpha_{n}$ est négatif en vertu de (2). On voit que l'on a $R_{m}<1$, sauf pour $z=0$.

Le premier théorème de $M$. Poincaré est donc démontré; son auteur l'énonce sous la forme suivante : a étant un nombre quelconque, le produit

$$
e^{\alpha z} \mathrm{~F}(z)
$$

tend vers zéro lorsque z augmente indéfiniment avec un argument déterminé, cet argument étant tel que $e^{-a z}$ tende vers zéro. Si l'on pose

on a

$$
a=\rho e^{i \varphi}, \quad z=r e^{i \theta},
$$

$$
\begin{aligned}
& \text { et } \quad a z=p r[\cos (\varphi+\theta)+i \sin (\varphi+\theta)] \\
& \quad\left|e^{\alpha z}\right|=e p r \cos (\varphi+\theta)=e^{-\alpha r}, \\
& \text { en posant } \\
& \quad \alpha=-\rho \cos (\varphi+\theta) .
\end{aligned}
$$

(') En effet, $\mathrm{P}_{m}$ est de la forme $e^{-k r} \varpi(z), \varpi(z)$ étant un polynome et $k$ un nombre positif; on a manifestement

$$
\left|\mathbf{P}_{m}\right|<e^{-k r} \mathbf{P}(r),
$$

$\mathrm{P}(r)$ étant un certain polynome en $r$; done $\left|\mathrm{P}_{m}\right|$ tend vers zéro lorsque $r$ augmente indéfiniment. 
L'hypothèse faite sur $e^{a z}$ entraìne que $a$ est positif; nous sommes ainsi ramené au théorème que nous avons démontré.

Considérons maintenant une fonction de genre $p$,

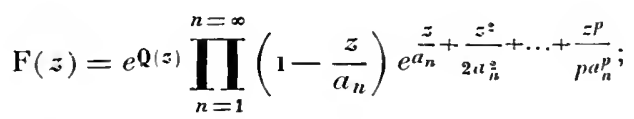

le polynome $\mathrm{Q}(z)$ est au plus de degré $p$ et la série

$$
\sum\left|\frac{1}{a_{n}^{p+1}}\right|
$$

est convergente. Nous allons prouver que, $r$ désignant le module de zet a étant un nombre positif arbitraire, le produit

$$
e^{-\alpha, p+1} \mathbf{F}(z)
$$

tend vers zéro en même temps que $\frac{\mathrm{I}}{\mathrm{r}}$.

Dans ce but nous allons d'abord faire voir que, le nombre $p$ étant donné, on peut déterminer un nombre $k$ tel que l'on ait, quel que soit $u$,

$$
\left|(1-u) e^{\frac{u}{1}+\frac{u^{q}}{2}+\ldots+\frac{u p}{p}}\right|<e^{k|u| p+1} .
$$

Remarquons d'abord que le produit

$$
e^{-1 u \mid p+1}(\mathrm{I}-u) e^{\frac{u}{1}+\frac{u^{2}}{2}+\ldots+\frac{u p^{p}}{p}}
$$

tend vers zéro lorsque $|u|$ augmente indéfiniment; il existe donc un nombre $\mathrm{R}$ tel que ce produit soit inférieur à l'unité lorsque $|u|$ dépasse $R$.

Considérons maintenant les valeurs de $u$ telles que $|u|$ soit compris entre $\frac{1}{2}$ et $\mathrm{R}$; soit $M$ le maximum du module du produit

$$
(1-u) e^{\frac{u t}{1}+\frac{u^{2}}{2}+\cdots+\frac{u^{p}}{p}}
$$

lorsque $u$ prend toutes ces valeurs; déterminons le nombre $k^{\prime}$ par la condition

$$
\mathbf{M}<e^{k \cdot\left(\begin{array}{l}
1 \\
2
\end{array}\right)^{p+1}} ;
$$

on aura évidemment, pour toutes les valeurs de $u$ considérées,

$$
\left|(1-u) e^{\frac{u}{1}+\frac{u^{2}}{2}+\ldots+\frac{u p}{p}}\right|<e^{u^{\prime} \mid u u^{p+1}}
$$


Enfin, supposons le module de $u$ inférieur à $\frac{1}{2}$; on a

$$
\log \left[(1-u) e^{\frac{u}{1}+\frac{u^{q}}{2}+\ldots+\frac{u p}{p}}\right]=-\frac{u^{p+1}}{p+1}-\frac{u^{p+2}}{p+1}-\ldots
$$

La valeur absolue de ce logarithme est donc inférieure à

$$
\frac{|u|^{p+1}}{p+1}\left(\mathrm{I}+|u|+\left|u^{2}\right|+\left|u^{3}\right|+\ldots\right)<\frac{|u| p+\mathrm{I}}{p+1} \frac{\mathrm{I}}{\mathrm{I}-|u|}<\frac{2|u|^{p+1}}{p+\mathrm{I}},
$$

et, puisque $p$ est au moins égale à $u n$, inférieure à $|u|^{p+1}$, on a donc encore, pour ces valeurs de $u$,

$$
\left|(1-u) e^{\frac{u}{1}+\frac{u^{2}}{2}+. .+\frac{u p}{p}}\right|<e^{|u|^{p+1}} .
$$

Il suffit donc, pour que l'inégalité

$$
\left|(1-u) e^{\frac{u}{1}+\frac{u^{2}}{2}+\ldots+\frac{u p}{p}}\right|<e^{k i n, p+1}
$$

soit vérifiée pour toute valeur de $u$, de prendre $k$ égal au plus grand des deux nombres $k^{\prime}$ et un (').

Ce point étant établi, reprenons la fonction $\mathrm{F}(z)$, et déterminons les nombres positifs $\alpha_{n}$ de manière que

$$
\alpha=\alpha_{1}+\alpha_{2}+\ldots+\alpha_{12}+\ldots,
$$

et que de plus, pour les valeurs de $n$ supérieures à un nombre fixe $m$, l'on ait

$$
\alpha_{n}=k\left|\frac{1}{a_{n}^{p+1}}\right|,
$$

$k$ étant le nombre que nous venons de définir. Cela est possible, puisque la série

$$
\sum\left|\frac{1}{a_{n}^{p+1}}\right|
$$

est convergente. Le nombre $m$ étant fixé, nous décomposerons $e^{-\alpha, r^{p+1}} \mathrm{~F}(z)$ en deux facteurs; nous poserons

$$
\mathrm{P}_{m}=e^{Q(z)} \prod_{n=1}^{n=m} e^{-\alpha_{n} \cdot p+1}\left(1-\frac{z}{a_{n}}\right) e^{\frac{\tilde{z}}{a_{n}}+\ldots+}+\frac{z p}{p a_{n}^{p}}
$$

( $\left.{ }^{1}\right)$ On vérifierait aisément que l'on a $k^{\prime}>1$, mais ce point est ici sans importance. 
et

$$
\mathrm{R}_{m}=\prod_{n=m+1}^{n=\infty} e^{-\alpha_{n} r r^{p+1}}\left(1-\frac{z}{a_{n}}\right) e^{\frac{z}{a_{n}}+\ldots+\frac{z p}{p a_{n}^{p}}} .
$$

Il résulte de la manière dont $x_{n}$ a été choisi que tous les facteurs de $\mathbf{R}_{m}$ ont un module inférieur à l'unité et, d'autre part, il est visible que le produit $\mathrm{P}_{m}$ tend vers zéro lorsque $r$ augmente indéfiniment, car il est de la forme

$$
\bar{\sigma}(\tilde{z}) e^{\left.\varpi_{1}(z)-c\right) \cdot p+1},
$$

$\varpi(z)$ et $\varpi_{1}(z)$ étant des polynomes, ce dernier de degré au plus égal à $p$, et $c$ une constante positive.

Donc le produit $\mathrm{P}_{m} \mathrm{R}_{m}$, c'est-à-dire $e^{-\alpha r^{p+1}} \mathrm{~F}(z)$ tend vers zéro.

$$
\text { C. Q. F. D. }
$$

Tel est le premier des importants résultats dus à M. Poincaré; il nous sera commode de l'énoncer sous la forme suivante : nous désignerons par $\mathbf{M}(r)$ le maximum du module de la fonction entière $\mathbf{F}(z)$ lorsque le module de $z$ est égal (1) à $r$. Lorsque la fonclion $\mathrm{F}(z)$ est de genre $p$, lon a

$$
\mathbf{M}(r)<e^{\alpha, p+1}
$$

quel que soit le nombre positif. $\alpha$, pourvu que $r$ soit assez grand. Nous exprimerons aussi ce fait en disant que la fonction positive croissante $\mathbf{M}\left(r^{\cdot}\right)$ est inférieure à $e^{\alpha \cdot r+1}$, ou que l'ordre de grandeur de $\mathbf{M}(r)$ est inférieur à cclui de $e^{\alpha_{r+1}+1}$.

Que peut-on conclure de ces résultats pour le développement en série de $\mathrm{F}(z)$; nous poserons

$$
\mathrm{F}(z)=\mathbf{A}_{0}+\mathrm{A}_{1} z+\mathbf{A}_{2} z^{2}+\ldots+\mathbf{A}_{m} z^{n i}+\ldots,
$$

et nous considérerons l'intégrale, prise suivant un chemin réel,

$$
\mathrm{J}(z)=(p+\mathrm{I}) \int_{0}^{\infty} e^{-r p+1} \mathrm{~F}(r z) r^{\prime h} d r,
$$

$\iota$ étant un nombre positif arbitraire. Il est manifeste que cette intégrale a un sens, quel que soit z, car, quel que soit $x$, on a, a partir d'une certaine valeur de $r$,

$$
|\mathbf{F}(r z)|<e^{\left.\alpha|z|\right|^{p+1}|r|^{p+1}}
$$

et lorsque $|z|$ est donné, on pcut prendre $\alpha$ de manière que $\alpha|z|^{p+1}$ 
soit inférieur à un; l'intégrale a dès lors, à partir d'une certaine valeur de $r$, ses éléments inférieurs en module à ceux de l'intégrale convergente :

$$
\int_{0}^{\infty} e^{-n \cdot p^{+1}} r^{\prime \prime} d r, \quad \eta_{i}>0
$$

L'intégrale $\mathbf{J}(z)$ représente donc une fonction analytique de $z$ dépourvue de singularités à distance finie, c'est-à-dire une fonction entière. Or on obtient, en remplaçant $\mathrm{F}(r z)$ par son développement en série,

avcc

$$
\mathrm{J}(z)=\mathrm{B}_{0}+\mathrm{B}_{1} z+\ldots+\mathrm{B}_{m} z^{m}+\ldots
$$

$$
\mathrm{B}_{m}=\mathrm{A}_{m} \int_{0}^{\infty}(p+\mathrm{I}) e^{-r^{p+1}} r^{m+h} d r=\Lambda_{m} \Gamma\left(\frac{m+h+1}{p+\mathrm{I}}\right),
$$

comme on s'en assure en faisant $r^{p+1}=t$. On a posé, suivant l'usage,

$$
\mathrm{r}(x)=\int_{0}^{\infty} e^{-t} t^{x-1} d t .
$$

Mais $\mathbf{J}(z)$ étant une fonction entière, $\mathbf{B}_{m}$ tend vers zéro lorsque $m$ augmente indéfiniment. On obțient ainsi la seconde proposition de M. Poincaré : la fonction $\mathbf{F}(z)$ étant de genre $p$, le produit

$$
\Lambda_{m} \Gamma\left(\frac{m+h+1}{p+1}\right)
$$

tend vers zéro lorsque $m$ augmente indéfiniment. On peut même, comme l'a fait observer M. Hadamard, affirmer que la racine $m^{\text {ième }}$ de ce produit tend vers zéro.

On peut donner des formes un peu différentes au théorème de M. Poincaré; pour ne rien emprunter à la théorie de la fonction $\Gamma(x)$, nous remarquerons simplement que, d'après (1), cette fonction croît avec $x$ et que, $m$ étant entier, on a

$$
\Gamma(m+\mathbf{I})=m !
$$

Cela étant, considérons le produit $(m a) !, m$ et $a$ étant deux entiers, on a visiblement

$(m a) !=1,2,3, \ldots, \quad a(a+1) \ldots(m a-1) m a<a^{a}(2 a)^{a}(3 a)^{a} \ldots(m a)^{a}$, puisque l'on a ainsi remplacé chacun des $a$ premiers facteurs par le plus grand d'entre eux, $a$; chacun des $a$ suivants par le plus grand d'entre eux, $2 a$, etc. 
On a donc

$$
(m a) !<a^{m a}(m !)^{a},
$$

ou bien

$$
[(m a) !]^{\frac{1}{\prime \prime}}<a^{m} m !
$$

$\mathrm{Or}$

$$
m !=\mathbf{\Gamma}(m+\mathbf{r})
$$

on a donc, a fortiori, si $x$ est un nombre supérieur à $m$,

$$
[(m a) !]^{\frac{1}{a}}<a^{m} \Gamma(x+1) .
$$

Soient maintenant $\mu$ un entier quelconque, ma le multiple de $a$ immédiatement supérieur à $\mu$; on a $m a-\mu<a$, et l'on obtient, $\mu$ élant plus grand que $m$,

$$
(\mu !)^{\frac{1}{a}}<[(m a) !]^{\frac{1}{a}}<a^{m} \Gamma(x+1)<a \mu^{\mu} \Gamma(x+1) .
$$

D’ailleurs $x$ est un nombre quelconque supérieur à $m$; done $a x$ est supérieur à $a m$ et, par suite, à $\mu+a$; c'est-à-dire que $x$ est supérieur à $\frac{\mu+a}{a}$.

Cela posé, remarquons que la fonction $F(z)$ étant une fonction de genre $p$, il en est de même de $\mathrm{F}(a z)$; nous pouvons donc remplacer $A_{\mu}$ par $a^{\mu} A_{\mu}$ et affirmer que, quel que soit $a$, le produit

$$
a^{\mu} \mathbf{A}_{\mu} \Gamma\left(\frac{\mu+h+1}{p+1}\right)
$$

tend vers zéro lorsque $\mu$ croît indéfiniment; nous prendrons $a=p+\mathbf{I}$ et $h=2 p+\mathbf{1} ;$ dès lors

$$
\frac{\mu+h+1}{p+\mathrm{I}}=\frac{\mu+a}{a}+\mathrm{I},
$$

et l'on a

$$
a^{\mu} \Gamma\left(\frac{\mu+h+I}{p+1}\right)>[\mu !]^{\frac{1}{p+1}}
$$

Donc le produit

$$
A_{\mu}(\mu !)^{\frac{1}{p+}}
$$

tend vers zéro lorsque $\mu$ augmente indéfiniment (1); telle est

( $\left.{ }^{1}\right)$ On aurait pu voir que la racine $\mu^{i \grave{m} \text { de }}$ ce produit tend vers zéro. 
la forme que l'on peut donner au second théorème fondamental de M. Poincaré; on peut dire aussi que l'on a, à partir d'une certaine valeur de $\mu$,

$$
\left|A_{m}\right|<\frac{1}{\sqrt[p+1]{m !}} .
$$

En particulier, pour une fonction de genre $p=0$, on a, à partir d'une certaine valeur de $m$,

$$
\left|\mathbf{A}_{m}\right|<\frac{\mathbf{I}}{m !} .
$$

Nous verrons, dans le Chapitre suivant, quels importants compléments a apportés M. Hadamard aux propositions de M. Poincaré, en en démontrant les réciproques; nous allons terminer ce Chapitre en montrant comment l'introduction de la notion de l'ordre, à la place de celle du genre, permet d'obtenir des inégalités plus précises que celles de M. Poincaré.

\section{Le module maximum des fonctions d'ordre p.}

Considérons un produit de facteurs primaires de genre $p>0$, sans facteur exponentiel; c'est ce que nous appellerons un produit canonique de facteurs primaires,

$$
\mathrm{F}(z)=\prod_{n=1}^{n=\infty}\left(1-\frac{z}{a_{n}}\right) e^{\frac{z}{a_{n}}+\frac{z^{2}}{2 a_{n}^{a}}+\ldots+\frac{z^{p}}{p a_{n}^{p}}}
$$

Nous savons que, $r_{n}$ désignant le module de $a_{n}$, la série

$$
\sum \frac{1}{r_{n}^{p+1}}
$$

est convergente; nous supposerons, de plus, que l'on sache que la série

$$
\sum \frac{1}{r_{n}^{a}}
$$

est convergente, $\sigma$ étant un nombre inférieur à $p \nmid-1$. Nous allons montrer que, $\varepsilon$ étant un nombre positif arbitraire donné à l'avance, l'inégalité

$$
|\mathbf{F}(\boldsymbol{z})|<e^{\xi r^{n}}
$$


est vérifiée pour toutes les valeurs de $r$ dépassant un nombre fixe. On voit que si l'on fait $\sigma=p+\mathrm{I}$, c'est-à-dire si l'on suppose seulement la convergence de la série

$$
\sum \frac{1}{r_{n}^{p+1}+1}
$$

notre proposition se réduit à celle de $M$. Poincaré; il est inutile de la démontrer de nouveau dans ce cas; nous supposerons done essentiellement $p-1-\sigma$ positif et non nul. Nous supposerons aussi $\sigma-p$ positif.

La série (1) à termes positifs déeroissants étant convergente, nous pouvons, le nombre positif $\eta$ étant donné à l'avance, trouver un nombre $m$ tel que l'inégalité

entraîne

$$
n>m
$$

$$
r_{n}>\eta n^{\frac{1}{\sigma}}
$$

D'autre part, nous savons qu'il existe un nombre $k$ tel que l'on ait, quel que soit $u$,

$$
\left|(1-u) e^{\frac{u}{1}+\frac{u^{2}}{2}+\ldots+\frac{u^{p}}{p}}\right|<e^{k \mid u^{p+1}} \mid .
$$

Cela posé, désignons par $r$ le module de $z$ et déterminons le nombre $n$ par la condition

$$
r=\eta n^{\frac{1}{\sigma}}
$$

Le nombre $n$ étant donné d'avance, nous avons pris $r$ assez grand pour que l'on ait $n>m$.

Nous allons, pour évaluer le module de $F(z)$, considérer d'abord le produit des $n$ premiers facteurs primaires, puis le produit des facteurs restants.

Considérons d'abord le produit

$$
\mathrm{P}_{\imath}=\prod_{i=1}^{i=n}\left(1-\frac{z}{a_{i}}\right) e^{\frac{z}{i_{i}}+\frac{z^{z}}{2 a_{i}^{a}}+\ldots+\frac{z^{p}}{p a_{t}^{p}}} .
$$

Nous savons que l'on a

$$
\left|1-\vec{z}_{a_{i}}\right|<e^{\left|\frac{z}{a_{i}}\right|}=e^{\frac{r}{r_{i}}} .
$$


Il en résulte

$$
\left|\mathrm{P}_{n}\right|<\prod_{i=1}^{i=n} e^{\frac{2 r}{r_{i}}+\frac{r^{2}}{2 r_{i}^{i}}+\ldots+\frac{r^{p}}{p r^{p}}}
$$

c'est-à-dire

$$
\left|\mathrm{P}_{n}\right|<e^{2 r \sum_{1}^{n} \frac{1}{r_{i}}+\frac{r^{2}}{2} \sum_{1}^{n} \frac{1}{r_{i}^{2}}+\ldots+\frac{r^{p}}{p} \sum_{1}^{n} \frac{1}{r_{i}^{p}} .}
$$

Nous allons ehereher une limite supérieure de la somme

$$
\sum_{1}^{n} \frac{\mathrm{I}}{r_{i}^{k}}
$$

Nous savons que, pour $n>m$, l'on a

$$
r_{n}>\eta_{1} n^{\frac{1}{\sigma}}
$$

II cn résulte

$$
\sum_{1}^{n} \frac{1}{r_{i}^{\lambda}}<c_{\lambda}+\frac{\mathrm{I}}{r_{i} \lambda} \sum_{2}^{n} \frac{\mathrm{I}}{n^{\frac{\lambda}{\sigma}}}
$$

en désignant par $c_{\lambda}$ une constante positive indépendante de $n$. Cette constante dépend d'ailleurs de $n$, de même que $m$. On a, d'ailleurs,

$$
\sum_{2}^{n} \frac{\mathrm{I}}{n^{\frac{\lambda}{\sigma}}}<\int_{1}^{n} \frac{d n}{n^{\frac{\lambda}{\sigma}}}<\frac{\sigma}{\sigma-\lambda} n^{\frac{\sigma-\lambda}{\sigma}} .
$$

Comme on a

$$
n^{\frac{1}{\sigma}}=\frac{r}{r_{1}}
$$

il en résulte, $\sigma--\lambda$ étant positif,

$$
\sum_{1}^{n} \frac{1}{r_{i}^{\lambda}}<c_{\lambda}+\frac{1}{r_{i} \lambda}\left(\frac{r}{n}\right)^{\sigma-\lambda} \frac{\sigma}{\sigma-\lambda} .
$$

Done

$$
r \sum_{1}^{n} \frac{1}{r_{i}^{\lambda}}<c_{\lambda} r^{\lambda}+\frac{r^{\sigma}}{r_{i}^{\sigma}} \frac{\sigma}{\sigma-\lambda},
$$

et enfin

$$
\left|\mathrm{P}_{n}\right|<e^{2\left(c_{1} r+\frac{r^{\sigma}}{n^{\sigma}} \frac{\sigma}{\sigma-1}\right)+\frac{1}{2}\left(c_{2} r^{2}+\frac{r^{\sigma}}{n^{\sigma}} \frac{\sigma}{\sigma-2}\right)+\ldots+\frac{1}{p}\left(c_{p} r p+\frac{r^{\sigma}}{\eta^{\sigma}} \frac{\sigma}{\sigma-p}\right)} .
$$


Donnons-nous maintenant d'avance un nombre $\varepsilon$; nous choisirons le nombre $n$ de manière que l'on ait

$$
\frac{1}{\gamma_{0} \sigma}\left[\frac{2 \sigma}{\sigma-1}+\frac{\sigma}{2(\sigma-2)}+\frac{\sigma}{3(\sigma-3)}+\ldots+\frac{\sigma}{\mu(\sigma-p)}\right]<\varepsilon ;
$$

le nombre $\eta$ étant ainsi choisi, les constantes $c_{\lambda}$ seront déterminées; nous prendrons $r$ assez grand (') pour que l'on ait

$$
2 c_{1} r+\frac{1}{2} c_{2} r^{2}+\ldots+c_{p} r^{p}<\varepsilon r^{\sigma},
$$

et l'on aura dès lors

$$
\left|P_{n}\right|<e^{2 \varepsilon r^{\sigma}} .
$$

Il nous reste à trouver une limite supérieure du module du produit

$$
\mathbf{R}_{n}=\prod_{n+1}^{\infty}\left(1-\frac{z}{a_{i}}\right) e^{\frac{\tilde{z}}{\bar{a}_{i}}+\ldots+\frac{z p}{p u_{i}^{p}}}
$$

Or nous avons vu que le module de chaque facteur de ce produit est inférieur à

$$
e^{k\left|\tilde{a}_{i}\right|^{p+1}}=e^{k\left|\frac{r}{r_{i}}\right|^{p+1}} .
$$

On a donc

$$
\left|\mathbf{R}_{n}\right|<e^{k r^{p+1}} \sum_{n+1}^{\infty} \frac{1}{r_{i}^{p+1}}
$$

Pour évaluer la somme

$$
\sum_{n+1}^{\infty} \frac{1}{r_{i}^{p+1}}
$$

nous remarquerons que $n$ étant supérieur à $m$, on a toujours ici

Il en résulte

$$
r_{i}>n_{i} \frac{1}{\bar{\sigma}}
$$

$$
\sum_{n+1}^{\infty} \frac{1}{r_{i}^{p+1}}<\int_{n}^{\infty} \frac{d n}{r_{i}^{p+1} \frac{p+1}{n^{\frac{p}{\sigma}}}}=\frac{\mathrm{I}}{r_{i}^{p+1}} \frac{\sigma}{p+1-\sigma} \frac{\mathrm{T}}{n^{\frac{p+1}{\sigma}-\sigma}},
$$

( ${ }^{1}$ De plus, on prend $r>r_{m}$, le nombre $m$ étant déterminé d'après le choix de $r_{i}$. 
ou, en remplaçant $n^{\frac{1}{\sigma}}$ par $\frac{r}{\gamma_{1}}$,

$$
\left|\mathbf{R}_{n}\right|<e^{k r^{p+1}} \frac{1}{n p^{p+1}} \frac{\sigma}{p+1-\sigma}\left(\frac{n}{r}\right)^{\frac{p+1-\sigma}{\sigma}},
$$

c'est-à-dire

$$
\left|\mathrm{R}_{n}\right|<e^{\frac{k \sigma}{p+1-\sigma} \frac{1}{n^{\sigma}} r^{\sigma}} \text {. }
$$

Mais la constante $l i$ ne dépend ni de $n$ ni de $r$; nous pouvons donc supposer que $n$ a été choisi de telle sorte que l'on ait

et il cn résulte

$$
\frac{k \sigma}{p+1-\sigma} \frac{1}{r_{i} \sigma}<\varepsilon
$$

$$
\left|\mathrm{R}_{\|}\right|<e^{\varepsilon r^{\sigma}} .
$$

On a donc

$$
|\mathrm{F}(z)|=\left|\mathrm{P}_{n} \mathrm{R}_{n}\right|<e^{2 \varepsilon r^{\sigma}},
$$

ce que nous voulions obtenir (1).

Soit maintenant o l'exposant de convergence de la suite

$$
r_{1}, \quad r_{2}, \ldots, r_{n}, \ldots,
$$

et supposons d'abord que $p$ ne soit pas entier. Nous distinguerons deux cas :

1" La série

$$
\sum \frac{1}{r_{n}^{2}}
$$

est divergente; nous prendrons $\sigma=\rho+\varepsilon, \varepsilon$ étant arbitrairement petit et nous aurons l'inégalité (2)

$$
|F(z)|<e^{r+\varepsilon}
$$

(') Nous avons supposé $\sigma>1$; si l'on a $\sigma<\mathbf{1}$, déterminons un nombre entier $a$ tel que $a \sigma>1$ et posons $z=y^{a}$; on a $\mathrm{F}(z)=\Phi(y) ; \Phi(y)$ s'exprime par un produit eanonique de facteurs primaires (voir p. 28). Si l'on désigne par $\mathbf{R}_{n}$ les modules des zéros de $\Phi(y)$ la série $\sum \frac{\mathrm{I}}{\mathrm{R}_{n}^{a \sigma}}$ est convergente, on a donc

$$
|\Phi(y)|<e^{\varepsilon \mathbf{R}^{a \sigma}}
$$

$R$ élant le module de $y$; mais $\mathrm{R}^{a}=r$; done

$$
|\mathbf{F}(z)|<e^{\varepsilon r^{*}} \text { C. Q. F. D. }
$$

(2) Il est inutile d'écrire $|\mathrm{F}(z)|<e^{\varepsilon^{\prime} r^{p+\varepsilon}}$, puisque $\varepsilon$ est arbitrairement petit. 
qui sera vérifiée, quel que soit $\varepsilon$ donné à l'avance à partir d'une certaine valeur de $r$.

$2^{\circ}$ La série

$$
\sum \frac{1}{r_{n}^{p}}
$$

est convergente; nous pourrons alors prendre $\sigma=\rho$ et affirmer que l'on a, pour $r$ assez grand,

$$
|\mathrm{F}(z)|<e^{\varepsilon \cdot r^{p}},
$$

quel que soit le nombre $\varepsilon$ donné d'avance.

Supposons maintenant que $\rho$ soit entier; si l'on est dans le premier cas, nous prendrons encore $\sigma=p+\varepsilon$; et l'on aura encore l'inégalité (I); si l'on est dans le second cas, nous aurons l'inégalité (2), en vertu du théorème de M. Poincaré.

Pour résumer les résultats obtenus, nous conviendrons de dire que, dans le premier cas, $p$ est l'ordre par défaut et, dans le second, que $\rho$ est l'ordre par excès ('). On a alors l'énoncé suivant :

La fonction $\mathrm{F}(z)$ étant d'ordre $p$, on a toujours, pour $r$ assez grand,

$$
|\mathrm{F}(z)|<e^{r^{p^{+\varepsilon}}}
$$

si, de plus, on sait que p est l'ordre par excès, on a aussi

$$
|\mathrm{F}(z)|<e^{\varepsilon r^{q}}
$$

quel que soit a donné d'avance, pourvu que $r$ soit assez grand $(\stackrel{*}{*})$.

(1) On montrera aisément que dans ce cas on peut trouver une série de nombres positifs $\varepsilon_{n}$ tendant vers zéro tels que la série $\sum \frac{\mathrm{I}}{r_{n}^{p-\xi_{n}}}$ soit convergente.

( $)$ J'ai donné la première partie de ce théorème, avec des indications sur la démonstration, dans mon Mémoire sur les zéros des fonctions entières. Une démonstration complète en a élé donnée par M. von Schaper dans le Mémoire que nous avons déjà cité. Cette première partie pourrait se ramener au théorème de M. Poincaré par le procédé indiqué dans la note de la page précédente; il suffirait de prendre $a$ successivement égal aux dénominateurs des réduites du développement de $\rho$ en fraction continue.

La deuxième partie est nouvelle. 
Le module maximum et la fonction majorante.

Nous venons de démontrer des inégalités auxquelles satisfait le module maximum ( $\left.{ }^{1}\right) \mathbf{M}(r)$ d'une fonetion d'ordre $\rho$; eherehons quelles conséquences en résultent pour les coeffieients.

Nous savons que si l'on pose

$$
\mathbf{F}(z)=\mathbf{\Lambda}_{0}+\mathbf{A}_{1} z+\ldots+\mathbf{\Lambda}_{m} z^{m}+\ldots,
$$

on a, le eontour $\mathrm{C}$ entourant l'origine,

$$
\mathrm{A}_{m}=\frac{1}{2 i \pi} \int_{\mathrm{C}} \frac{\mathrm{F}(z) d z}{z^{m+1}},
$$

d'où, en prenant pour $\mathrm{C}$ un eerele de rayon $r$,

$$
\left|A_{m}\right|<\frac{M(r)}{r^{m}} .
$$

Supposons que l'on ait $\mathbf{M}(r)<e^{r^{\sigma}}$; on en conelut

$$
\left|A_{m}\right|<\frac{e^{r^{p}}}{r^{m}} \text {. }
$$

Nous allons déterminer $r$ de manière que le second membre soit aussi petit que possible; nous aurons ainsi l'inégalité la plus précise que l'on puisse obtenir au moyen de(1). Or, en annulant la dérivée de $\frac{e^{r^{\sigma}}}{r^{m}}$ par rapport à $r$, on obtient

$$
\sigma r^{\sigma-1}-\frac{m}{r}=0,
$$

c'est-à-dire $r^{\sigma}=\frac{m}{\sigma}$, et l'on constate aisément que cette valeur de $r$ fournit effectivement un minimum de l'expression considérée. Nous obtenons ainsi

$$
\left|\Lambda_{m}\right|<\frac{e^{\frac{m}{\sigma}}}{\left(\frac{m}{\sigma}\right)^{\frac{m}{\sigma}}}, \quad \text { ou bien } \quad \frac{\mathrm{I}}{\sqrt[m]{\left|\mathrm{A}_{m}\right|}}>\left(\frac{m}{e \sigma}\right)^{\frac{1}{\sigma}}=\mathrm{K} m^{\frac{1}{\sigma}} .
$$

( $\left.{ }^{1}\right)$ Nous entendons toujours par là module maximum pour $|z|=r$. 
Nous nous contenterons de ce résultat; on le préciserait un peu par l'emploi des formules d'approximation de la théorie de la. fonction $\Gamma$; mais nous n'en avons pas besoin.

Nous préférons nous étendre sur un point fort important : quelle est la précision que l'on peut espérer de la méthode par. laquelle nous avons obtenu une limite supérieure de $\left|\mathbf{A}_{m}\right|$; en d'autres termes, est-ce que $\left|A_{m}\right|$ est notablement inférieur à celte limite supérieure?

Une remarque préliminaire est indispensable pour bien poser la question : il est aisé de s'assurer qu'une fonction entière peut croître aussi vite que l'on veut et avoir cependant une infinité de coefficients nuls : par exemple, tous les coefficients de rang impair. Il ne peut donc s'agir pour nous de trouver une limite inférieure de $\left|\mathrm{A}_{m}\right|$ et de montrer qu'elle diffère peu de la limite supérieure adoptée ('). Mais on peut se placer à un point de vue différent; nous avons des inégalités de la forme

$$
\left|\mathrm{A}_{m}\right|<\mathrm{B}_{m},
$$

les $B_{m}$ étant des nombres positifs; posons

$$
\mathrm{H}(r)=\mathrm{B}_{0}+\mathrm{B}_{1} r+\mathrm{B}_{2} r^{2}+\ldots+\mathrm{B}_{m} r^{m}+\ldots
$$

Si nous prouvons que la croissance de la fonction $\mathrm{H}(r)$ diffère peu de la croissance de la fonction $\mathbf{M}(r)$, nous pourrons dire que les inégalités (2), considérées dans leur ensemble, sont suffisamment précises. Nous reviendrons d'ailleurs sur ces remarques à la fin de ce paragraphe; nous pourrons alors leur donner plus de netteté.

Considérons une fonction entière arbitraire

$$
\mathbf{F}(z)=\Lambda_{0}+\Lambda_{1} z+\ldots+\Lambda_{m} z^{m}+\ldots,
$$

et désignons par $\mathbf{A}(r)$ le maximum des valeurs positives de la partie réelle de $\mathrm{F}(z)$. Nous avons, si l'on pose $A_{0}=\alpha_{0}+i \alpha_{0}^{\prime}$,

$$
r^{m}\left|\Lambda_{m}\right| \leqq\left\{\mathrm{A}(z)-2 x_{0} .\right.
$$

( ${ }^{1}$ ) Ce serait un intėressant sujet de recherehes que la détermination des coefficients $A_{m}$ qu'il faut nécessairement supposer différents de zéro lorsqu'on donne la fonction $\mathrm{I}(r)$; on prouverait aisément, par exemple, que si lous les $\mathrm{A}_{m}$ sont nuls, excepté ceux dont le rang est $m^{m^{m}}$, la fonction $M(r)$ ne peut ètre prise arbitrairement. 
Les inégalités (3) sont analogues aux inégalités (1); nous allons considérer, d'une manière générale, les inégalités ( ')

$$
\left|\mathrm{A}_{m}\right|<\frac{\mathrm{S}(r)}{r^{m}}
$$

$\mathrm{S}(r)$ étant une fonction positive croissante, et en déduire une limite supérieure du module de $\mathrm{F}(z)$. Nous appellerons fonction majorante relative à $\mathrm{F}(z)$ la fonction positive croissante suivante:

$$
\mathfrak{h l}(r)=\left|\Lambda_{0}\right|+\left|\Lambda_{1}\right| r+\ldots+\left|\Lambda_{m}\right| r^{m}+\ldots
$$

Nous allons déduire des inégalités (4) une inégalité importante à laquelle satisfait la fonction $\mathfrak{M} \mathfrak{M}(r)$. Désignons par $h$ un nombre positif quelconque; nous avons, en utilisant les inégalités (4),

$$
\begin{aligned}
\mathfrak{A l}(r-h)<\mathrm{S}(r) & +\frac{\mathrm{S}(r)}{r}(r-h)+\frac{\mathrm{S}(r)}{r^{2}}(r-h)^{2}+\ldots \\
& +\frac{\mathrm{S}(r)}{r^{m}}(r-h)^{m}+\ldots,
\end{aligned}
$$

c'est-à-dire, en remarquant que $1+\frac{r-h}{r}+\frac{(r-h)^{2}}{r^{2}}+\ldots=\frac{r}{h}$,

$$
\mathfrak{n}(r-h)<\frac{r \mathrm{~S}(r)}{h}
$$

On obtiendrait de la mème manière l'inégalité

$$
\mathfrak{A l}^{\prime}(r-h)<\frac{r \mathrm{~S}(r)}{h^{2}}
$$

et des inégalités analogues pour les dérivées successives. Malgré leur grande importance, nous nous contentons de les signaler en passant, n'en ayant pas besoin ici. Revenons à l'inégalité (5).

Supposons, par exemple, que l'on ait

$$
\mathrm{S}(r)<e^{\varepsilon r^{?}},
$$

et prenons $h=\mathbf{I}$; nous obtiendrons

$$
\mathfrak{m}(r-\mathbf{I})<r e^{\varepsilon r^{p}}
$$

( $\left.{ }^{1}\right)$ L'inégalité (3) n'est vérifiée que si $m$ est différent de zéro; nous pouvons supposer les inégalités (4) vérifiées pour toute valeur de $r$, tout en supposant que $S(r)$ coïncide avec $4 A(r)-2 \alpha_{0}$ à partir d'une certaine valeur de $r$. 
c'est-à-dire

$$
\operatorname{At}(r)<(r+1) e^{\varepsilon(r+1)^{e}}<e^{\varepsilon^{\prime} r^{2}},
$$

en désignant par $\varepsilon^{\prime}$ un nombre arbitraire supérieur à $\varepsilon$. Donc si $\mathrm{S}(r)$ vérifie l'inégalité (6), quel que soit $\varepsilon$ donné d'avance (pourvu que $r$ soit assez grand), il en sera de même de $\mathfrak{A}(r)$.

Ainsi, dans ce cas particulier, l'inégalité (5) fournit immédiatement pour $\mathfrak{A} \mathfrak{A}(r)$ la même limitation supéricure que pour $\mathrm{S}(r)$. Nous allons arriver à un résultat un peu moins précis, mais néanmoins de grande importance, sans rien supposer sur $\mathrm{S}(r)$. Rappelons que $\mathrm{S}(r)$ est une fonction positive croissante et que $\frac{\mathrm{S}(r)}{r^{m}}$ augmente indéfiniment avec $r$, quel que soit le nombre $m$ donné à l'avance.

Cela posé, supposons que l'on ait, lorsque $r$ est compris entre $r_{0}$ et $r_{0}+h$, l'inégalité

$$
\mathfrak{M}(r)=\cdot[\mathrm{S}(r)]^{1+\alpha},
$$

\% étant un nombre positif. L'inégalité (5) pent s'écrire, en remplaçant $r$ par $r_{0}+h$, puis $h$ par $\frac{h}{2}$

$$
\mathfrak{m}\left(r_{0}\right)<\frac{\left(r_{0}+\frac{h}{2}\right) \mathrm{S}\left(r_{0}+\frac{h}{2}\right)}{\frac{h}{2}} ;
$$

d'où, en tenanl compte de ( $/)$,

$$
\left[\mathrm{S}\left(r_{0}\right)\right]^{1+x}<\frac{\left(r_{0}+\frac{h}{2}\right) \mathrm{S}\left(r_{0}+\frac{h}{2}\right)}{\frac{h}{2}},
$$

c'est-à-dire

$$
\mathrm{S}\left(r_{0}+\frac{h}{2}\right)>\frac{\frac{h}{2}}{r_{0}+\frac{h}{2}}\left[\mathrm{~S}\left(r_{0}\right)\right]^{1+x} .
$$

On aurait de même, d'après (

$$
\mathfrak{m}\left(r_{0}+\frac{h}{2}\right)<\frac{\left(r_{0}+\frac{h}{2}+\frac{h}{4}\right) \mathrm{S}\left(r_{0}+\frac{h}{2}+\frac{h}{4}\right)}{h},
$$

I3. 
et, en tenant compte de ( 7 ) puis de (8),

$$
\begin{aligned}
\mathrm{S}\left(r_{0}+\frac{h}{2}+\frac{h}{4}\right) & >\frac{\frac{h}{4}}{r_{0}+\frac{h}{2}+\frac{h}{4}}\left[\mathrm{~S}\left(r_{0}+\frac{h}{2}\right)\right]^{1+\alpha} \\
& >\frac{\frac{h}{4}\left(\frac{h}{2}\right)^{1+\alpha}}{\left(r_{0}+\frac{h}{2}+\frac{h}{4}\right)\left(r_{0}+\frac{h}{2}\right)^{1+\alpha}}\left[\mathrm{S}\left(r_{0}\right)\right]^{(1+\alpha)^{2}} .
\end{aligned}
$$

On obtiendra de même

$$
\begin{aligned}
& \mathrm{S}\left(r_{0}+\frac{h}{2}+\frac{h}{4}+\frac{h}{8}\right) \\
& >\frac{\frac{h}{8}\left(\frac{h}{4}\right)^{1+\alpha}\left(\frac{h}{2}\right)^{\left(1+\alpha^{2}\right.}}{\left(r_{0}+\frac{h}{2}+\frac{h}{4}+\frac{h}{8}\right)\left(r_{0}+\frac{h}{2}+\frac{h}{4}\right)^{(1+\alpha)}\left(r_{0}+\frac{h}{2}\right)^{\left(1+\alpha_{1}^{2}\right.}}\left[\mathrm{S}\left(r_{0}\right)\right]^{(1+\alpha)^{3}} .
\end{aligned}
$$

En remarquant que l'on a

$$
r_{0}+\frac{h}{2}+\frac{h}{4} \div \frac{h}{8}+\ldots+\frac{h}{2^{n}}<r_{0}+h,
$$

oll obtient

$$
\begin{aligned}
& \mathrm{S}\left(r_{0}+\frac{h}{2}+\frac{h}{4}+\frac{h}{8}\right) \\
& \quad>\left(\frac{h}{r_{0}-i-h}\right)^{1+(1+\alpha)+(1+\alpha)^{2}} \frac{1}{2^{3+2(1+\alpha)+(1+\alpha)^{2}}}\left[\mathrm{~S}\left(r_{0}\right)\right]^{1+\alpha !^{3}} .
\end{aligned}
$$

On aura, en général,

$$
\begin{aligned}
& \mathrm{S}\left(r_{0}+\frac{h}{2}+\frac{h}{4}+\ldots+\frac{h}{2^{n}}\right) \\
& =\left(\frac{h}{r_{0}+h}\right)^{1+(1+\alpha)+\ldots+(1+\alpha)^{n-1}} \frac{1}{2^{n+(n-1)(1+\alpha)+\ldots+(1+\alpha)^{n-1}}}\left[\mathrm{~S}\left(r_{0}\right)\right]^{(1+\alpha)^{n}} .
\end{aligned}
$$

Or on a

$$
\begin{aligned}
& 1+(1+\alpha)+\ldots+(1+\alpha)^{n-1}=\frac{(1+\alpha)^{n}-1}{\alpha}<\frac{(1+\alpha)^{n}}{\alpha}, \\
& n+(n-1)(1+\alpha)+\ldots+(1+\alpha)^{n-1} \\
& \quad=(1+\alpha)^{n-1}\left[1+\frac{9}{1+\alpha}+\frac{3}{(1+\alpha)^{2}}+\ldots+\frac{n}{(1+\alpha)^{n-1}}\right]
\end{aligned}
$$


et

$$
\mathrm{I}+\frac{9}{\mathrm{I}+\alpha}+\frac{3}{(\mathrm{I}+\alpha)^{2}}+\cdots+\frac{n}{(1+\alpha)^{n-1}}<\frac{(1+\alpha)^{2}}{\alpha^{2}}
$$

puisque

$$
\frac{(1+x)^{2}}{\alpha^{2}}=\sum_{1}^{\infty} \frac{n}{(1+x)^{n-1}}
$$

Donc

$$
\mathrm{S}\left(r_{0}+\frac{h}{2}+\ldots+\frac{h}{2^{n}}\right)>\left[\left(\frac{h}{r_{0}+h}\right)^{\frac{1}{\alpha}}\left(\frac{1}{2}\right)^{\frac{1+\alpha}{\alpha^{2}}} \mathrm{~S}\left(r_{0}\right)\right]^{(1+\alpha)^{n}}
$$

Posons

$$
\left(\frac{h}{r_{0}+h}\right)^{\frac{1}{\alpha}}\left(\frac{1}{2}\right)^{\frac{1+\alpha}{\alpha^{2}}} \mathrm{~S}\left(r_{0}\right)=\mathrm{P}
$$

on aura, la fonction $S(r)$ étant croissante,

$$
\mathrm{S}\left(r_{0}+h\right)>\mathrm{S}\left(r_{0}+\frac{h}{2}+\ldots+\frac{h}{2^{n}}\right)>\mathrm{P}^{(1+x)^{n}} .
$$

Si $\mathrm{P}$ est plus grand que $u n, \mathrm{~S}\left(r_{0}+h\right)$ dépasse toute quantité assignable, ce qui est absurde. On a donc nécessairement

$$
\mathrm{P}<\mathrm{I} \text {. }
$$

Ainsi l'hypothèse que l'inégalité

$$
\mathfrak{a l}(r)>[\mathrm{S}(r)]^{1+\alpha}
$$

est vérifiée en même temps que les inégalités

$$
r_{0} \leqq r<r_{0}+h
$$

entraîne

$$
\left(\frac{h}{r_{0}+h}\right)^{\frac{1}{\alpha}}\left(\frac{1}{2}\right)^{\frac{1+\alpha}{\alpha^{2}}} \mathrm{~S}\left(r_{0}\right)<1 .
$$

On peut tirer de cette inégalité d'intéressantes conséquences relativement à l'étendue des intervalles dans lesquels peut être vérifiée l'inégalité $(7)$; en modifiant un peu la démonstration, on prouve même aisément que, si $r_{1}$ est un nombre supérieur à $r_{0}$ et si, dans l'intervalle $r_{0}-r$, l'inégalité (7) est vérifiée dans des intervalles dont l'étendue dépasse $h$, on a

$$
\left(\frac{h}{r_{1}}\right)^{\frac{1}{\alpha}}\left(\frac{\mathrm{I}}{2}\right)^{\frac{1+\alpha}{\alpha^{2}}} \mathrm{~S}\left(r_{0}\right)<1
$$


Mais nous nous contenterons de démontrer le résultat suivant: Quelque petit que soit le nombre a donné d'avance, l'inégalité $(\sigma)$ ne peut être vérifiée pour toutes les valeurs de r qui dépassent un nombre fixe; car, s'il en était ainsi, on pourrait choisir $r_{0}$ et $h$ de manière que l'inégalité (9) ne soit pas vérifiée. En d'autres termes, on a pour une infinité de valeurs de $r$ dépassant toute limite

$$
\mathfrak{h l}(r)<[\mathrm{S}(r)]^{1+x} .
$$

Or " nous pouvons prendre $\mathrm{S}(r)=4 \mathrm{~A}(r)-2 a_{0}$; comme le nombre $\alpha$ est arbitraire, nous pouvons écrire simplement

$$
\mathfrak{n l}(r)<[\Lambda(r)]^{1+x} .
$$

Cette inégalité est véritiée pour une infinité de valeurs de $r$ dépassant toute limite (1). On a d'ailleurs manifestement, quel que soit $r$,

$$
\mathrm{A}(r) \leqq \mathbf{M}(r) \leqq \mathfrak{M}(r) .
$$

Les inégalités (10) et (11) constituent la relation que nous voulions établir entre les fonctions $\mathrm{A}(r)$ et $\mathfrak{A M}(r)$. Il est clair que l'on a aussi, en désignant par $\mathrm{B}(\boldsymbol{r})$ le maximum des valeurs positives de la partie réelle de $-\mathrm{F}(z)$,

$$
\mathrm{B}(r) \leqq \mathrm{U}(r) \leqq \mathfrak{A}(r)<[\mathrm{B}(r)]^{1+x},
$$

et l'on en conclut, $\propto$ étant toujours un nombre positif arbitraire, que les inégalités

$$
[\Lambda(r)]^{1-\alpha}<\mathrm{B}(r)<\left[\Lambda\left({ }^{-}\right)\right]^{1+\alpha}
$$

sont vérifiées dans les mêmes conditions que l'inégalité (10). Nous conviendrons de dire que ces inégalités (12) exprimentque les fonctions $\mathrm{A}(r)$ et $\mathrm{B}(r)$ sont du même ordre de grandeur. Nous omettrons les commentaires qu'appellerait celte définition dans le cas général $\left({ }^{2}\right)$; contentons-nous d'observer que si l'on a

$$
\mathbf{A}(r)<e^{\varepsilon r^{?}},
$$

( $\left.{ }^{1}\right)$ On pourrail préciser davantage en utilisant l'inégaiité (9) comme nous l'arous indiqué.

$\left({ }^{2}\right)$ Voir le Mémoire déjà cité : Sur les zèros des fonctions entières (Acla mallematica, t. X.I, p. 368 et suiv.). 
on pcut en conclure (p. 64)

$$
\mathfrak{m}(r)<e^{z^{\prime} r^{2}},
$$

$\varepsilon^{\prime}$ différant aussi peu que l'on veut de $\varepsilon \mathrm{et}$, par suite, pouvant être pris arbitrairement petit dans le cas où il en est ainsi pour $\varepsilon$. Ainsi la limitation trouvée pour $\mathfrak{A l}(r)$ est ici la même que celle qu'on s'était donnée pour $\mathbf{A}(\boldsymbol{r})$ ou tout au moins (dans le cas où $\varepsilon$ est fixe) s'en rapproche autant que l'on veut. Or $\mathfrak{A l}(r)$ est certainement supérieur à $\mathrm{A}(\boldsymbol{r})$; on ne pouvait donc trouver pour $\mathfrak{A}(r)$ une limitation inféricure à celle qui est dounée pour $\mathrm{A}(r)$; donc, on peut dire que la limitation trouvée pour $\mathbf{A} \mathfrak{A}(\boldsymbol{r})$, si elle n'est pas la meilleure possible, est du moins très près de l'être. Or, on l'a obtenue en remplaçant les $\left|\boldsymbol{A}_{\boldsymbol{m}}\right|$ par les limites supérieures que fournissent les inégalités (3) ou (4) (pages 63 et 64); donc ces limites supérieures sont elles-mêmes trc̀s près d'être les meilleures possibles.

Une remarque est cependant nécessaire; considérons, pour fixer les idées, l'inégalité

$$
\left|\Lambda_{m}\right|<\frac{M(r)}{r^{m}}
$$

le second membre renferme le nombre positif arbitraice $r$; on aura la limitation la meilleure que puisse fournir cette inégalité en recherchant le minimum de $\frac{M(r)}{r^{m}}$, comme nous l'avons fait plus haut dans un cas particulier.

Mais, en fait, ce n'est pas ainsi que nous avons procédé pour avoir une limile supérieure de $\mathbf{A l l}(r-h)$; nous avons donné à $r$ une valeur fixe, indépendante de $m$ (mais non de $r-h$ ); comment avons-nous pu ainsi arriver à un résultat qui ne soit pas absolument grossier, alors que, si $r$ est fixe, les expressions $\frac{M(r)}{r^{m}}$ décroissent comme une simple progression géométrique, c'est-à-dirc bien moins rapidement que les coefficients d'une fonction entière? Voici l'explication de ce paradoxe apparent : lorsqu'on donne à z dans $F(z)$ une valeur de module assez grand $r$, les termes vont d'abord en croissant, pour décroître ensuite très rapidement; le rang $m$ des termes les plus grands croît, d'ailleurs, évidemment avec $r$; si l'on cherche une limite supérieure du module de $F(z)$, 
on pourra avoir un résultat assez approché si l'on remplace les coefficients $A_{m}$ de ces termes les plus grands par des valeurs assez approchées, même si l'on commet de très grandes erreurs sur les coefficients $A_{m}$ de rang très élevé, à condition que ces erreurs n'empêchent pas ces termes de rang très élevé de rester petits. C'est ce que nous avons fait en réalité; on voit que le fait que la valeur trouvée pour $\mathfrak{M A}(r)$ est assez approchée, pour une valeur donnée de $r$, prouve simplement que les valeurs de certains coefficients $A_{m}$ sont assez approchées; mais comme le rang $m$ de ces coefficients dépend de $r$, il est légitime de dire que les valeurs limites des $\left|A_{m}\right|$ sont assez approchées dans leur ensemble si, pour toute valeur de $r$, on a unc limite supérieure assez approchée de $\mathfrak{A M}(r)$.

On remarquera que, lorsque l'on prend approximativement

$$
\left|\mathrm{A}_{m}\right|=\frac{\mathbf{M}(r)}{r^{m}},
$$

cela revient à prendre

$$
\mathbf{M}(r)=\left|\mathbf{A}_{m}\right| r^{m}=\left|\mathbf{A}_{m} z^{m}\right|,
$$

e'est-à-dire à supposer que le module maximum de la fonction $\mathrm{F}(z)$ est égal au module d'un de ses termes. On pent induire de ce qui précède que, si ce terme est convenablement choisi, l'erreur relative ainsi commise est très faible; c'est cc qu'il est très aisé de vérifier sur des exemples simples; nous ne nous attarderons pas à prouver que, d'une manière tout à fait générale, on commet une erreur relative très faible en prenant pour $\mathbf{M}(r)$ le plus grand des modules des termes successifs $\mathbf{A}_{m} z^{m}$ de $\mathrm{F}(\bar{z})$. 


\title{
CHAPITRE IV.
}

\author{
LES RËSULTATS DE II. IIADAMARD.
}

\section{Le premier théorème de M. Iladamard.}

Dans un Mémoire fondamental, couronné en 1892 par l'Académie des Sciences et publié en $189^{3}$ dans le Journal de Mathématiques, M. Hadamard a fait faire à la théorie des progrès essentiels ct ouvert en même temps la voie à des recherches nouvelles. Une étude complète de ce Mémoire cxcéderait les limites de ces Lecons; nous nous bornerons aux fonctions de genre fini, pour lesquelles les démonstrations se simplifient beaucoup ("). Peut-être aurons-uous l'occasion, dans de nouvelles Leçons, d'étudier d'autres parties du Mémoire de M. Hadamard.

Le premier des théorèmes de M. Hadamard peut être considéré: comme la réciproque d'une proposition démontrée dans le Chapitre précédent. Nous avons trouvé une limite supérieure de la croissance d'un produit de facteurs primaires, connaissant son ordre réel, ou, ce qui revient au même, l'exposant de convergence de la suite des modules de ses zéros. Nous allons apprendre maintenant, étant donnée une limite supérieure de la croissance d'unc fonction entière, à déterminer une limite inférieure de l'exposant de convergence de la suite de ces zéros.

Pour arriver à ce résultat, nous ne suivrons pas la voie par laquelle M. Hadamard l'a obtenu; une méthode plus simple, due à $\mathrm{I}$. Schou, permet d'établir un résultat équivalent à celui de 11. Hadamard, lorsqu'on se borne aux fonctions de genre

(1) Dans le Hémoire déjà cité, M. von Schaper donne aux fonctions de genre fini le nom de Hadamard'schen Functionen. Il nous parait que c'est restreindre la portée des travaux de M. Hadamard, dont les résultats principaux s'appliquent ou s'étendent sans peine aux fonctions de genre infini. 
fini ('). La méthode de M. Hadamard se raltache à ses belles recherches sur la détermination des points singuliers d'une fonction définie par une série de Taylor, et son exposition trouvcrait plutôt sa place dans des Leçons consacrées à cette importante question.

Le problème qu'il s'agit actuellement de résondre est le suivant :

On donne une fonction entière et l'on sait que son module maximum $\mathrm{M}(r)$ (pour $|z|=r)$ est inférieur à une fonction connue; on demande d'en conclure une limite supérieure du nombre des zéros dont le module est inférieur à un nombre quelconque $r$.

Voici la solution de M. Schou $\left({ }^{2}\right)$; comme nous l'avons dit, la limite qu'elle fournil est équivalente à celle qu'a obtenue M. Hadamard dans lc cas des fonctions de genre fini; elle est moins bonne dans le cas où le genre devient infini.

Désignons par $\mathrm{F}(z)$ une fonction entière et par $\mathrm{M}(r)$ le maximum de son module pour $|z|=r$; nous supposons que la fonction $\mathrm{M}(r)$ vérifie, pour toute valeur de $r$, l'inégalité

$$
\mathbf{M}(r)<e^{\mathbf{V}(r)},
$$

$\mathrm{V}(r)$ étant une fonction donnée.

Pour plus de précision, nous supposerons que la fonction $\mathrm{F}(z)$ ne s'annule pas pour $z=0$; on peut alors admettre qu'on a multiplié $\mathrm{F}(z)$ par un facteur convenable, de manière à avoir la relation

$$
\mathbf{F}(\mathrm{o})=1 .
$$

Désignons par $a_{1}, a_{2}, \ldots, a_{n}, \ldots$ les zéros de $\mathrm{F}(z)$ rangés par ordre de modules croissants (ou du moins non décroissants); chaque zéro figure dans cette suite un nombre de fois égal à son ordre de multiplicité. Posons

$$
\mathrm{P}(z)=\left(a_{1}-z\right)\left(a_{2}-z\right) \ldots\left(a_{n}-z\right)
$$

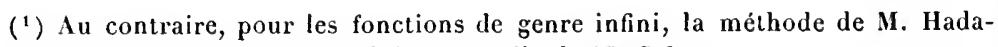
mard permet d'aller bien plus loin que celle de M. Schou.

( $\left.{ }^{2}\right)$ Comptes rendus, t. CXXV, p. 763 . 
le quotient $\frac{F(z)}{P(z)}$ est une fonction entière; on a donc, C étant un contour simple entourant l'origine,

$$
\frac{\mathrm{F}(0)}{\mathrm{P}(0)}=\frac{\mathrm{I}}{a_{1} a_{2} \ldots a_{n}}=\frac{1}{2 i \pi} \int_{\mathrm{C}} \frac{\mathrm{F}(z)}{z \mathrm{P}^{\prime}(z)} d z .
$$

Nous prendrons pour $\mathrm{C}$ un cercle de rayon $r$ égal à $s r_{n}, r_{n}$ étant le module de $a_{n}$ et $s$ un nombre plus grand que deux; on a dès lors, sur ce cercle,

$$
|\mathrm{P}(z)|>\left(r-r_{1}\right)\left(r-r_{2}\right) \ldots\left(r-r_{n}\right)>\left(r-r_{n}\right)^{n}=(s-1)^{n} r_{. "}^{\prime \prime}
$$

On en conclut, en se servant de (1),

$$
\left|\frac{1}{2 i \pi} \int_{\mathrm{C}} \frac{\mathrm{F}(z) d z}{z \mathrm{P}(z)}\right|<\frac{e^{\mathrm{V}(m)}}{r_{n}^{\pi}(s-1)^{n}} .
$$

D'autre part, les modules des $a$ ne décroissant pas,

$$
\left|\frac{\mathrm{I}}{a_{1} a_{2} \ldots a_{n}}\right|>\frac{\mathrm{I}}{r_{n}^{\prime \prime}}
$$

L'inégalité (2) devient alors

d'o ì

$$
\frac{\mathrm{I}}{r_{h}^{n}}<\frac{e^{r \cdot r)}}{r_{h}^{\prime \prime}(s-1)^{n}},
$$

$$
n \log (s-1)<V(r) .
$$

$\mathrm{Or}$, nous avons posé $r=s r_{n}$, c'est-à-dire

$$
r_{n}=\frac{r}{s}
$$

l'inégalité (3) donne donc une limite supérieure du nombre $n$ des racines inférieures à $\frac{r}{s} ; s$ y désigne un nombre quelconque supérieur à deux.

Nous allons appliquer la formule (3) au cas où I'on a

$$
\mathrm{V}(r)=\mathrm{A} r \boldsymbol{x}
$$

A et $\propto$ étant des constantes; nous obtenons

$$
n<\mathrm{B},{ }_{\mu}^{x},
$$


en posant

$$
\mathrm{B}=\frac{\mathrm{A} s^{x}}{\log (s-1)}
$$

L'inégalité (õ) s'écrit aussi

$$
r_{n}>\mathrm{C}_{1} n^{\frac{1}{x}}
$$

C étant, ainsi que $\mathrm{B}$, une constante.

Nous dirons que la fonction $\mathrm{F}(z)$ est d'ordre apparent $\rho^{\prime}$ si, quelque petit que soit $\varepsilon$, on a, à partir d'une certaine valeur. de $r$,

$$
\mathbf{U}(\boldsymbol{r})<e^{r^{\mathrm{i}^{\prime+\varepsilon}}} .
$$

On peut, par suile, choisir la constanle A de manière que l'on ait pour loute valeur de $r$

$$
\mathrm{M}(r)<e^{.1 r^{\varepsilon^{\prime}+\varepsilon}}:
$$

on a, dès lors, pour toute valeur de $n$, l'inégalité (6), avec $x=\rho^{\prime}+\varepsilon ;$ c'est dire que l'exposant de convergence $\rho$ de la suite des rn est au plus égal à p’; car la séric

$$
\sum \frac{1}{1 \cdot p+2 \xi}
$$

est visiblement convergente, en vertu de $(6)$, et le nombre $\varepsilon$ cst arbitraire.

Tel est lc premier théorème de M. Hadamard, lorsqu'on le borne aux fonctions de genre fini et qu'on utilise les définitions que nous avons introduiles: L'ordre réel $p$ d'une fonction entière est au plus égal ì son ordre apparent p'.

Ce point établi, soit $F(z)$ une fonction entière d'ordre apparent $\rho^{\prime}$; nous désignons par $a_{1}, a_{2}, \ldots, a_{n}, \ldots$ ses zéros; nous ponvons former un produit canonique de facteurs primaires

$$
\mathrm{G}(z)=\prod_{1}^{\infty}\left(1-\frac{z}{a_{n}}\right) e^{\frac{z}{a_{n}}+\frac{-2}{2 a_{n}^{2}}+\ldots+-\frac{\partial p}{p a p}}
$$

ayant les mèmes zéros que $\mathrm{F}(z)$; bien entendu, nous prenons le nombre $p$ aussi petit que possible; $p$ est dès lors nécessairement inférieur à $\rho$ et, par suite, à $\rho^{\prime}$, sauf peut-être dans le cas où $\rho^{\prime}$ est enticr; on peut alors avoir

$$
p=\rho=\rho^{\prime} .
$$


Dans tous les cas, l'ordre apparent de $\mathrm{G}(\tilde{z})$ est égal à $\rho$, comme nous l'avons vu dans le Chapitre précédent (1). Or nous avons

$$
\mathrm{F}(z)=e^{\mathrm{H}(z)} \mathrm{G}(z) ;
$$

nous nous proposons de prouver que l'ordre apparent de $e^{\mathbf{u}(z)}$ est au plus égal à $p^{\prime}$, c'est-à-dire que $\mathbf{H}(z)$ est un polynome de degré au plus égal à $\rho^{\prime}$. Pour cela, nous ferons voir qu'en multipliant un produit canonique tel que $\mathrm{G}(z)$, dont l'ordre pest inférieur ou égal à $\rho^{\prime}$, par un facteur $e^{\mathbf{u}_{(z)}}$ d'ordre apparent supéricur à $\rho^{\prime}$, on obtient une fonction entière d'ordre apparent supérieur à $\rho^{\prime}$. Ce sera une conséquence immédiate du deuxième théorème de $\mathbf{M}$. Hadamard, auquel est consacré le paragraphe suivant. Si nous admettons provisoirement ce résultat, nous voyons que l'ordre apparent de $\mathbf{F}(z)$ étant $\rho^{\prime}$, celui de $e^{\mathbf{I l}(z)}$ ne peut dépasser $\rho^{\prime}$. Si le nombre $\rho^{\prime}$ n'est pas entier, le degré $q$ de $\mathbf{H}(z)$ est nécessairement inféricur à $\rho^{\prime}$. Or l'ordre apparent du produit $e^{\mathbf{l l}(z)} \mathrm{G}(z)$ ne peut dépasser le plus grand des deux nombres $q$ et $p$, ordres apparents des deux facteurs. Donc, dans ce cas, on a nécessairement

$$
\rho^{\prime}=\rho \text {. }
$$

Ainsi, lorsque l'ordre apparent $\rho^{\prime}$ n'est pas entier, l'ordre réel p lui est égal; le degré $q$ du polynome $\mathbf{H}(z)$ qui figure dans le facteur exponentiel est, d'ailleurs, inférieur à l'ordre.

Dans le cas où le nombre $\rho^{\prime}$ est entier, $q$ peut être égal à $q^{\prime} \mathrm{et} \rho$ inférieur à $\rho^{\prime}$; nous nous bornerons à affirmer que : le genre de la fonction est au plus égal à $\rho^{\prime}$; nous reviendrons sur ce cas dans le Chapitre V, consacré au théorème de M. Picard.

\section{Le deuxième théorème de M. Hadamard.}

Nous allons maintenant démontrer le théorème sur lequel nous venons de nous appuyer: $E n$ multipliant un produit canonique

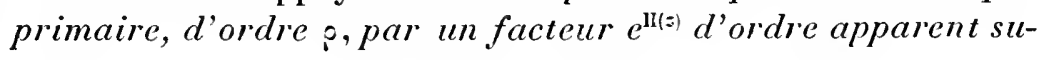

(1) Nous avons démontré seulement qu'il est au plus égal à $\rho$; mais, s'il était égal à un nombre $\rho^{\prime}<\rho$, nous nous trouverions en contradiction avec le théorème de $M$. Hadamard; nous pouvons dire que $\rho$ est l'ordre de $\mathrm{G}(\approx)$, sans qu'il soit besoin d'ajouter les mots apparent ou réel. 
périeur à $p$, on obtient une fonction entière d'ordre apparent supérieur à $p$. C'est une conséquence du deuxième théorème de M. Hadamard, qui s'énonce comme il suit :

Étant donnés un produit canonique $\mathrm{G}(z)$ de facteurs primaires d'ordre p, et un nombre positif arbitraire $\varepsilon$, on peut trourer une infinité de cercles de rayons indéfiniment croissants sur chacun desquels on a l'inégalité

$$
|\mathrm{G}(z)|>e^{-r^{2+\varepsilon}} .
$$

Nous allons démontrer d'abord ce théorème en supposant le nombre p inférieur à l'unité; nous l'étendrons ensuite aisément au cas où $p$ est quelconque. Soil donc

$$
\mathrm{G}(z)=\prod\left(1-\frac{z}{u_{n}}\right)
$$

une fonction entière d'ordre inférieur à un. Nous désignons par $r_{\|}$ le module de $a_{n}$ et nous supposons que la séric

$$
\sum \frac{1}{r \cdot j}
$$

est convergente, $\sigma$ étant un nombre inféricur à un. Il en résulte que l'on a ( $\left.{ }^{1}\right)$, à partir d'une certaine valeur de $n$,

$$
r_{n}>n^{\frac{1}{\sigma}} \text {. }
$$

Nous nous proposons de trouver des cercles sur lesquels on puisse déterminer un minimum du module de $\mathrm{G}(z)$; nous allons, pour cela, exclure le voisinage des points $a_{\|}$; on verra aisément quelles modifications pourraient ètre apportées à la démonstration, notamment en ce qui concerne l'épaisseur des couronnes dont il va être question, pour préciser encore davantage les résultats.

Traçons deux cercles (de centre $z=0$ ) ayant pour rayons respectifs

$$
r_{n}-\mathrm{I}, \quad r_{n}+\mathrm{I}
$$

ces deux cercles comprennent une couronne $\mathrm{C}_{n}$ d'épaisseur égale à deux. L'épaisseur totale des couronnes $\mathrm{C}_{1}, \mathrm{C}_{2}, \ldots, \mathrm{C}_{n}$ est

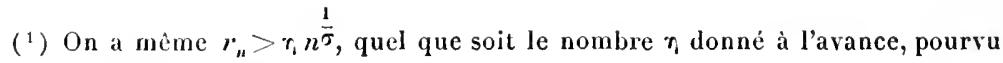
que $n$ soit assez grand. 
donc $2 n$; il résulte des hypothèses faites que le rapport de eette épaisseur à $r_{n}$ tend vers zéro lorsque $n$ augmente indéfiniment; eette remarque nous sera utile plus loin.

Pour démontrer le théorème de $\mathrm{M}$. Hadamard, il nous suffit d'observer qu'il existe des cercles $\mathrm{C}$ de centre $\mathrm{O}$ de rayons indéfiniment croissants, extérieurs à toutes les couronnes $\mathrm{C}_{n}$. Si nous supposons z situé sur un de ces eereles $C$, on aura visiblement, quel que soit $n$, d'après la définition même des eouronnes $\mathrm{C}_{n}$,

$$
\left|z-a_{n}\right|>1 \text {. }
$$

Il est dès lors aisé de trouver une limite inférieure du module du produit

$$
\mathrm{G}(z)=\prod\left(1-\frac{z}{a_{n}}\right) ;
$$

désignons par $r$ le module de $z$; déterminons le nombre $m$ par les eonditions

$$
r_{m} \leqq 2 r \leqq r_{m+1}
$$

et le nombre $n$ par les conditions

$$
n^{\frac{1}{5}} \leqq 2 r<(n+1)^{\frac{1}{5}} .
$$

Nous supposerons $n$ assez grand pour que l'inégalité (1) soit vérifiée; il suffit que $r$ ait été lui-même pris assez grand. On a alors

$$
r_{n+1}>(n+1)^{\frac{1}{2}}>2 r \geqq r_{m}
$$

et, par suite,

Nous éerirons

$$
n \geqq m .
$$

$$
G(z)=\Lambda B C,
$$

en posant

$$
\begin{aligned}
& \mathrm{A}=\prod_{i=1}^{i=m}\left(1-\frac{z}{a_{i}}\right), \\
& \mathrm{B}=\prod_{i=m+1}^{i=n}\left(1-\frac{z}{a_{i}}\right), \\
& \mathrm{C}=\prod_{i=n+1}^{\infty}\left(1-\frac{\bar{a}}{a_{i}}\right) .
\end{aligned}
$$


Nous allons chercher suceessivement une limite inférieure pour chacun des modules des produits A, B, C.

On a

$$
\mathrm{A}=\prod_{i=1}^{i=m} \frac{\left(a_{i}-z\right)}{a_{i}}
$$

d'où, en vertu de (2) et de (3),

$$
|A|>\frac{1}{(2 r)^{m}} .
$$

Pour évaluer $B$, nous remarquerons que le module de $z$ étant $r$ et le module de $a_{i}$, pour $i>m$, étant supérieur à $2 r$, le module de chacun des facteurs de $B$ est supérieur à $\frac{1}{2}$; on a donc

$$
|\mathrm{B}|>\left(\frac{\mathrm{I}}{2}\right)^{n-m}
$$

Lnfin on a, en vertu de ( 1 ),

$$
\mid \mathrm{C}:>\prod_{i=n+1}^{\infty}\left(\mathrm{I}-\frac{r}{i^{\frac{1}{\sigma}}}\right) \text {. }
$$

Or, Jorsque $a$ est inférieır à $\frac{1}{2}$, on a l'inégalité

$$
(1-a)>e^{-2 a} .
$$

Donc

$$
\mid \mathrm{C}:>e^{-2 r} \sum_{i=n+1}^{\infty} i^{-\frac{1}{\sigma}}
$$

Or on a, $\sigma$ étant inférieur à un,

$$
\sum_{i=n+1}^{\infty} i^{-\frac{1}{\sigma}}<\int_{n}^{\infty} x^{-\frac{1}{\sigma}} d x=\frac{\sigma}{1-\sigma} n^{\frac{\sigma-1}{\sigma}}<\frac{\sigma}{1-\sigma}(2 r)^{\sigma-1},
$$

d'où, finalement,

$$
|\mathrm{C}|>e^{-\frac{\sigma}{1-\sigma}(2)^{\sigma}} .
$$

En multiplianı entre elles les inégalités obtenues, il vient

$$
|\mathrm{G}(\tilde{z})|=|\mathrm{ABC}|>e^{-\left[m \log r+n \log 2+\frac{\sigma}{1-\sigma}(2 r)^{\sigma}\right]} .
$$


L’inégalité (4) donne $m \leqq n<(2 r)^{\sigma}$, el, par suite,

$m \log r+n \log 2 \div \frac{\sigma}{1-\sigma}(2 r) \sigma<\rho\left(2^{\sigma} \log r+2^{\sigma} \log 2+2^{\sigma} \frac{\sigma}{1-\sigma}\right)$.

Or, quelque petit que soit le nombre donné à l'avance $\varepsilon$, on peut prendre le nombre $r$ assez grand pour vérifier l'inégalité

$$
2^{\sigma}\left(\log r+\log 2+\frac{\sigma}{1-\sigma}\right)<r^{5}
$$

on a, dès lors,

$$
\mathrm{G}(z) \mid>e^{-r^{\sigma+\varepsilon}}
$$

Cette inégalité est vérifiée sur une infinité de cercles de rayons indéfiniment croissants. Tel csi le résultat que nous voulions obtenir: c'est le second théorème de M. Hadamard. En effet, si la suite des $a_{n}$ a $\rho$ pour exposant de convergence, on peut prendre $\sigma=\rho+\varepsilon$, quelque petit que soil le nombre $\varepsilon$ donné d'avance, et l'inégalité (5) devient

$$
|\mathrm{G}(z)|>e^{-1,3+\hat{s}} \text {. }
$$

Nous avons supposé p inférieur à l'unité; supposons maintenant le nombre? plus grand que un et soit $q$ un entier supérieur à $\rho$. Soit toujours $\mathrm{G}(z)$ la fonction donnée et soit $\omega$ une racine primitive de l'équation

$$
\omega q=1 \text {. }
$$

Si nous posons

$$
\begin{gathered}
z q=y, \\
r q=\mathbf{R}=|y|, \\
\Phi(y)=\mathbf{F}(z)=\mathrm{G}(z) \mathrm{G}(\omega z) \ldots \mathrm{G}(\omega q-1 z),
\end{gathered}
$$

nous savons que la fonction $\Phi(y)$ a pour ordrc apparent $\frac{?}{q}$, c'est à-dire un nombre inférieur à un. Il y a donc une infinité de cercles $\mathrm{C}$ de rayons indéfiniment croissants, sur lesquels on a

$$
|\Phi(y)|>e^{-l^{2+\frac{2}{2}}}=e^{-r^{2+s},}
$$

quel que soit le nombre positif s donné d'avance. Mais nous avons vu dans le Chapitre précédent quc le nombre s étant donné, on a, pourvu que $r$ soit assez grand,

$$
|\mathrm{G}(\omega z)|<e^{r^{3+\varepsilon}}, \quad \ldots, \quad \mathrm{G}\left(\omega^{\prime} /-1 z\right)<e^{r^{2+\varepsilon}}, \quad \ldots
$$


Donc, sur les cercles C,

$$
\mathrm{G}(z)>\frac{e^{-r^{p+\varepsilon}}}{e^{r^{r+\varepsilon}} \cdot \frac{q}{2} \cdot e^{r^{p+\varepsilon}}}=e^{-q r^{?+\varepsilon}},
$$

ce qui est bien le résultat cherché, la présence du facteur $q$ étant indifférente, vu l'arbitraire $\varepsilon$.

Le théorème de $\mathrm{M}$. Hadamard est ainsi démontré dans tous les cas. Nous ne nous occuperons pas ici de l'extension possible de ce théorème aux fonctions de genre infini : nous préférons attirer l'attention sur ce fait que la démonstration employée permet de eompléter ce théorème, en ce qui concerne les rayons possibles des cercles $\mathrm{C}$ sur lesquels il s'applique.

Plaçons-nous d'abord, pour plus de netteté, dans le cas où p est inférieur à l'unité; figurons la partie positive de l'axe $\mathrm{O} x$ des quantités réelles et considérons les segments $\gamma_{n}$ qui correspondent aux couronnes $\mathrm{C}_{n}$

$$
r_{n}-1<x<r_{n}+1
$$

la longucur de chacun de ces segments est 2 ; si nous considérons les $n$ premiers, ils couvrent unc portion de la droite au plus égale à $2 n$ (elle est inférieure à $2 n$ dans le cas où les scgments empiètent les uns sur les autres). Mais dans le cas où nous nous trouvons, le rapport $\frac{2 n}{r_{n-1}}$ lend visiblement vers zéro lorsque $n$ augmente indéfiniment; donc, si nous eonsidérons sur $\mathrm{O} x$ un segment variable $\mathrm{OA}$, lorsque le point A s'éloigne indéfiniment, le rapport à $\mathrm{OA}$ de la somme des segments $\left(\gamma_{n}\right)$ intérieurs à $\mathrm{OA} \iota$ end vers séro. On peut prendrc pour extrémité du rayon d'un cercle $\mathrm{C}$ tout point extérieur aux segments $\gamma_{n}$ (el assez éloigné de $O$; mais cette dernière restriction est sans importance). Donc, lorsque A s'éloigne indéfiniment, la somme des segments silués sur. OA et clont les points peuvent être pris comme extrémités des rayons ales cercles $\mathrm{C}$ est telle que son rapport $\mathrm{OA}$ tend vers l'unité.

Indiquons tout de suite quelle peut être l'utilité de ces remarques. Supposons que nous ayons plusieurs fonctions entières. en nombre fini. Pour cliacune d'elles, le théorème de M. Hadamard nous apprend qu'il y a des cercles $\mathrm{C}$ sur lesquels l'inégalité (5) est vérifiéc; mais il n'est pas évident a priori qu'il y a 
des cercles $\mathrm{C}$ sur lesquels chaque fonction vérifie l'inégalité qui lui correspond; cependant, dans bien des démonstrations, la considération de tels cercles peut rendre de grands services. Les remarques qui viennent d'être faites nous prouvent qu'il en existe; car, si l'on a un nombre limité de fonctions entières, et si, pour eliacune d'elles, on marque les segments tels que $\gamma_{n}$, il est clair que l'étendue totale de tous ccux de ces segments qui sont intérieurs à $\mathrm{OA}$ continuera à être telle que son rapport à $\mathrm{OA}$ tende. vers zéro lorsque A s'éloigne indéfiniment : il y a donc une infinité de points s'éloignant indéfiniment, qui sont extérieurs à tous ces segments $\gamma_{n}$; les cercles $C$ passant par ces points sont tels que, sur chacun d'eux, l'inégalité de M. Hadamard est vérifiée pour toutes les fonctions données.

Le lecteur verra aisément que ces conclusions subsistent lorsque p est supérieur à l'unité; étant donné un nombre limité quelconque de fonctions entières de genre fini on peut trouver une infinité de cercles $\mathrm{C}$ de rayons indéfiniment croissants, sur lesquels l'inégalité de M. Hadamard est vérifiée pour chacune d'elles (').

On peut énoneer le théorème de M. Hadamard sous une forme plus expressive, en disant que, étant donnée une fonction entière, on peut trouver des cercles $\mathrm{C}$ sur lesquels son minimum est du même ordre de grandeur que l'inverse de son maximum. Mais, pour préciser cel énoneé, il serait nécessaire de développer une théorie des fonctions croissantes et des ordres de grandeur qui ne peut trouver place ici.

\section{Applications.}

Nous ne saurions indiquer toutes les applieations qui peuvent ètre laites des théorèmes de M. Hadamard; elles sont dejà nombreuses et le deviendiont sans doute plus encore. Nous allons nous contenter d'en emprunter deux, d'importance fort inégale, au Mémoire de M. Hadamard.

La premic̀re est d'un caractère tout à fait élémentaire; le lecteur

(1) J'ai indiqué sans démonstration ces complémerits au théorème de M. Hadamard dans mon llémoire Sur les zéros dées fonctions encières (Acta malhematica, t. XX, p. 361 ).

B. 
en imaginera sans peine de semblables. Posons

et

$$
z^{2}=y
$$

$$
\frac{\sin z}{z}=\mathbf{F}(y)
$$

$\mathbf{F}(y)$ est une fonction entière; et l'on a

$$
\mathbf{F}(y)=\frac{e^{i \sqrt{y}}-e^{-i \sqrt{y}}}{2 i \sqrt{y}}
$$

il en résulte immédiatement que, si $\mathbf{M}(r)$ désigne le maximum du module de $\mathrm{F}(y)$ pour $|y|=r$, on a

$$
e^{r^{\frac{1}{2}-\varepsilon}}<\mathbf{M}(r)<e^{r^{1}}
$$

en d'autres termes, l'ordre apparent de $\mathrm{F}(y)$ est égal à $\frac{1}{2}$. Or, les zéros de $\mathrm{F}(y)$ sont, comme on sait,

$$
y=\pi^{2}, \quad 4 \pi^{2}, 9 \pi^{2}, \ldots, \quad n^{2} \pi^{2}, \ldots,
$$

et l'on a, par suite, d'après Wcierstrass,

$$
\mathrm{F}(y)=e^{n(y)}\left(1-\frac{y}{\pi^{2}}\right)\left(1-\frac{y}{4 \pi^{2}}\right) \cdots\left(1-\frac{y}{n^{2} \pi^{2}}\right) \cdots,
$$

$\mathrm{H}(y)$ étant une fonction entière. Il résulte de ce qui précède (p. -j5) que cette fonction entière est une constance, laquelle est nulle, puisque $\mathrm{F}(y)$ est égal à un pour $y=0$. On obtient ainsi

$$
\mathrm{F}(y)=\prod_{1}^{\infty}\left(1-\frac{y}{n^{2} \pi^{2}}\right)
$$

c'est-à-dire

$$
\sin z=z \prod^{\infty}\left(1-\frac{z^{2}}{n^{2} \pi^{2}}\right)
$$

Cette formule, qu'Euler avait déjà obtenue par des procédés analyliques élémentaires, se trouve ainsi démontrée par une voie, à la vérité, plus détournée, mais sans aucun calcul. Si l'on utilisait seulement le résultat de Weicrstrass, sans connaître ceux de M. Hadamard, il faudrait, pour démontrer que la fonction entière II $(y)$ se réduit à une constante, un calcul à peu près aussi com- 
pliqué que pour établir directement la formule elle-même, par les méthodes de Cauchy ( 1 ).

La deuxième des applications est autrement importante; c'est pour y aboutir que M. Hadamard a été amené à entreprendre ses belles recherches : il s'agissait de déterminer le genre d'une fonction entière rencontrée par Riemann dans son célèbre Mémoirc sur les nombres premiers $\left({ }^{2}\right)$. Cette fonction entic̀re $\xi(t)$ est en relation étroite avec la fonction $\zeta(s)$ définie par la relation

$$
\zeta(s)=1+\frac{1}{2^{s}}+\frac{1}{3^{s}}+\ldots+\frac{1}{n^{s}}+\ldots
$$

Cette relation définit $\zeta(s)$ lorsque la partie réelle de $s$ est supérieure à un $\left({ }^{3}\right)$; mais la fonetion analytique ainsi obtenue peut être prolongée dans le reste du plan; voici comment Riemann en obtient une expression valable pour tout le plan.

On a identiquement, par la substitution $n x=y$,

$$
\int_{0}^{\infty} e^{-n x} x^{s-1} d x=\frac{1}{n^{s}} \int_{0}^{\infty} e^{-y} y^{s-1} d y .
$$

Si l'on pose, suivant l'usage,

$$
\mathbf{r}(s)=\int_{0}^{\infty} e^{-y} y^{s-1} d y,
$$

il vient

$$
\frac{\mathbf{\Gamma}(s)}{n^{s}}=\int_{0}^{\infty} e^{-n x} x^{s-1} d x
$$

el, par suite,

$$
\mathrm{Y}(s) \zeta(s)=\mathrm{\Gamma}(s) \sum_{1}^{\infty} \frac{1}{n^{s}}=\int_{0}^{\infty}\left(\sum_{1}^{\infty} e^{-n x}\right) x^{s-1} d x,
$$

c'est-à-dire

$$
\zeta(s)=\frac{\mathrm{I}}{\Gamma(s)} \int_{0}^{\infty} \frac{e^{-x} x^{s-1} d x}{1-e^{-x}}=\frac{1}{\Gamma(s)} \int_{0}^{\infty} \frac{x^{s-1} d x}{e^{x}-1} .
$$

( ${ }^{1}$ Voir, par exemple, le Cours d'Analyse de M. Hermite.

$\left.{ }^{2}\right)$ Ueber die Anzahl der Primzahlen unter einer gegebenen Grösse (Gesammelte Werke, p. $145-153$ de la $2^{\circ}$ édition).

$\left({ }^{3}\right)$ Bien entendu, lorsque $s=\alpha+i \beta$, on prend

$$
n^{s}=n^{\alpha}[\cos (\beta \log n)+i \sin (\beta \log n)],
$$

le logarithme ayant son sens arithmétique. 
Le chemin d'intégration, dans tout ce qui précède, est réel; la dernière intégrale n'a d'ailleurs un sens que si la partie réelle de $s-$ I est positive; c'est à cette condition que les calculs qui précèdent sont légitimes. Mais il est aisé de transformer l'expression analytique obtenue pour $\zeta(s)$, de manière à obtenir une nouvellc expression valable dans tout le plan.

Considérons dans ce but un contour ( $f i g$. I) défini comme il suit: deux parallèles infiniment voisines de l'axe réel et positif,

Fig. 1.

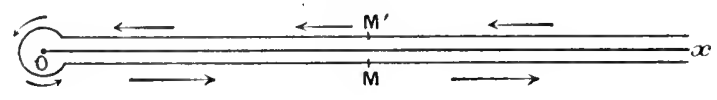

réunies par un petit cercle entourant l'origine; soil $\gamma$ ce contour; nous le supposons décrit dans le sens indiqué par les flèches: on va d'abord de l'infini jusqu'au voisinage de l'origime en passant au-dessus de l'axe des $x$, on tourne ensuite autour de l'origine dans le sens positif, et enfin on retourne à l'infini en restant au-dessous de $\mathrm{O} x$. Nous allous considérer, avec Riemann, l'intégrale

$$
\int_{\gamma} \frac{z^{s-1} d z}{e^{z}-1}
$$

mais il faut d'abord préciser la détermination que nous prenons pour $z^{s-1}$. On peut écrire

$$
z^{s-1}=e^{(s-1) l_{(1 ;} z} .
$$

$\mathrm{O}_{\mathrm{r}}$, sur les parties rectilignes du contour d'intégration, $z$ est très voisin de $\mathrm{O} x$; nous désignerons par $\log z$ la détermination du logarithme dont la partic imaginaire est très petite, c'est-à-dire ' pui deviendrait réelle si les parties rectilignes venaient se confondre avec $\mathrm{O} x$; nous prendrons, lorsque $z$ est en M, c'est-à-dire au-dessous de $\mathrm{O} x$,

$$
z^{s-1}=e^{(s-1) l_{0} z},
$$

et, dès lor's, on aura, en $\mathbf{M}^{\prime}$,

$$
z^{s-1}=e^{i s-1)(\log z-2 i \pi)}=e^{-2 i \pi s} e^{(s-1) \log z},
$$

tar on passe de $\mathbf{M}$ à $\mathbf{M}^{\prime}$ en tournant autour de l'origine dans le sens négatif. Si l'on suppose la partie réelle de $s-1$ positive, on 
ne change pas la valeur de l'intégrale en supposant que les parties rectilignes du contour d'intégration se confondent avec $\mathrm{O} x$, le rayon du petit cercle devenant $\mathrm{nul}$; on obtient ainsi

$$
\int_{\gamma} \frac{z^{s-1} d z}{e^{z}-1}=e^{-2 i \pi s} \int_{\infty}^{0} \frac{x^{s-1} d x}{e^{x}-1}+\int_{0}^{\infty} \frac{x^{s-1} d x}{e^{x}-1}
$$

On a donc

$$
\left(1-e^{-2 i \pi s}\right) \zeta(s)=\frac{\mathrm{I}}{\Gamma(s)} \int_{y} \frac{z^{s-1} d z}{e^{z}-1},
$$

et l'expression ainsi obtenue reste évidemment valable quel que soit $s$, puisque le contour d'intégration $\gamma$ ne passe pas par le point $z=0$.

Nous allons étudier l'intégrale

$$
\Phi(s)=\int_{\gamma} \frac{\tilde{z}^{s-1} d z}{e^{z}-1} ;
$$

c'est visiblement une fonction analytique de $s$, régulière pour toute valeur finie de $s$ : c'est donc une fonction entière. Nous allons déterminer son ordre apparent; pour cela, nous devons chercher une limite supérieure du module $\mathbf{M}(r)$ de $\Phi(s)$ pour $|s|=r$.

Dans ce but, divisons le contour $\gamma$ en deux parties $\gamma^{\prime}$ et $\gamma^{\prime \prime}$ comprenant respectivement les points pour lesquels on a

$$
|z|<r^{2} \quad \text { et } \quad z \mid>r^{2}
$$

on a, pour $|s|=r$,

$$
|\Phi(s)|<\left|\int_{\gamma^{\prime}} \frac{z^{s-1} d z}{e^{z}-1}\right|+\left|\int_{\gamma^{\prime}} \frac{z^{s-1} d z}{e^{z}-1}\right| .
$$

Or on voit aisément que l'on peut prendre $r$ assez grand pour que le second terme soit inférieur à un nombre donné d'avance (on ne doit pas oublier que le contour $\gamma^{\prime \prime}$ est aussi voisin que l'on veut de l'axe des quantités réelles et positives); quant au premier terme, il est inférieur à

$$
\left(r^{2}\right)^{r-1} \int_{\gamma}\left|\frac{d z}{e^{z}-1}\right|=A r^{2 r-2},
$$

A étant un nombre fixe; donc, ₹ ćtant un nombre arbitrairement 
petil donné d'avance, l'inégalité

$$
\mathrm{M}(r)<e^{r^{1+\varepsilon}}
$$

est vérifiée à partir d'une certaine valeur de r. Donc, l'ordre apparent de $\Phi(s)$ est au plus égal à un.

Ricmann considère la fonction

$$
\xi(l)=\Gamma\left(\frac{s}{2}\right) \frac{s}{2}(s-1) \pi-\frac{s}{2} \zeta(s)
$$

c:l posant

$$
s=\frac{1}{2}+i t
$$

il prouve que $\xi(t)$ est une fonction entière et, de plus, une fonclion paire, e'est-à-dire ne change pas lorsqu'on change $t$ en $-t$. Nous admettrons ces résultats, renvoyant pour la démonstration an Mémoire de Riemann. On a, en remplaçant $\zeta(s)$ par sa valeur,

$$
\zeta(t)=\frac{\Gamma\left(\frac{s}{2}\right) s(s-1) \pi^{-s} \Phi(s)}{\Gamma(s)\left(1-e^{-2 i \pi s}\right)} .
$$

Si nous observons que $\frac{1}{\Gamma(s)}$ est une fonction entière de $s$ d'ordre apparent égal à un, on voit que $\xi(t)$ se présente sous la forme

$$
\xi(t)=\frac{\mathrm{M}_{1}(t) \mathrm{M}_{2}(t) \ldots \mathrm{M}_{k}(t)}{\mathrm{N}_{1}(t) \mathrm{N}_{2}(t) \ldots \mathrm{N}_{h}(t)},
$$

$\mathrm{H}_{1}, \mathrm{M}_{2}, \ldots, \mathrm{M}_{k}, \mathrm{~N}_{1}, \ldots, \mathrm{N}_{h}$ étant des fonctions entières de 1 d'ordre apparent au plus égal à un (1).

Nous allons montrer que la fonction entière $\xi(t)$ est d'ordre apparent au plus ćgal à un. En effet, en remplaçant le numérateur cl le dénominateur par leur expression sous forme de produits de facteurs primaires, on a

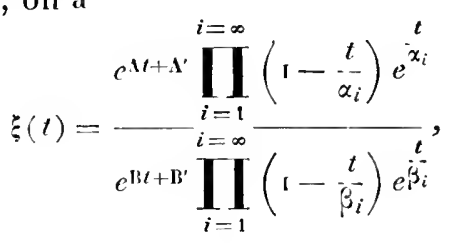

(') Il est à peine utile d'observer que l'ordre par rapport à $t$ est le mèmc que l'ordre par rapport à $s$, cn vertı de la rclation $s=\frac{1}{2}+i t$. 
l'exposant de convergence de la suite des $\alpha_{i}$ étant au plus égal à l'unité; comme $\xi(t)$ est une fonction entière, tous les $\beta_{i}$ figurent parmi les $\alpha_{i}$; en supprimant les facteurs primaires communs, il vient

$$
\xi(t)=e^{\mathrm{C} \ell+\mathrm{C}^{\prime}} \prod_{i=1}^{i=\infty}\left(1-\frac{t}{\gamma_{i}}\right) e^{\frac{t}{\gamma_{i}}}
$$

les $\gamma_{i}$ étant certains des $\alpha_{i}$; l'exposant de convergence de la suite dẹs $\gamma_{i}$ est donc au plus égal à l'unité; donc l'ordre de $\xi(t)$ est au plus égal à un. Mais nous avons dit que $\xi(t)$ est une fonction paire; si l'on prend $t^{2}$ comme variable, l'ordre de la fonction cntière de $t^{2}$ ainsi obtenue sera au plus égal à $\frac{1}{2}$, son genre sera donc nécessairement égal à zéro et l'on aura

$$
\xi(t)=\xi(0) \prod\left(1-\frac{t^{2}}{\gamma_{i}^{2}}\right) .
$$

Tel est le résultat fondamental de Riemann, dont M. Hadamard a été le premicr à donner une démonstration satisfaisante. Ce point étant rigoureusement établi, divers géomètres, parmi lesquels on doit citer surtout MM. von Mangoldt, de la Vallée-Poussin et Hadamard, ont pu en déduire d'importantes propriétés des fonctions $\xi(t)$ et $\zeta(s)$; mais nous devons nous contenter de ces brèves indications sur ce sujet difficile et qui continuera sans doute à attirer longtemps l'attention des géomètres (').

Nous avons tenu, en donnant cet exemple, à mettre en évidence les méthodes gènérales que l'on peut appliquer à l'étude des fonctions entières et nous avons laissé de côté ce qui a trait seulement aux propriétés particulières des fonctions considérées.

(') Pendant la correction des épreuves, j'ai connaissance d'un Mémoire de M. Jensen (Acta mathematica, t. XXII), Mémoire qui doit être suivi de plusieurs autres sur les fonctions entières et sur la fonction de Riemann. Je dois me contenter de signaler ces publications, dont limportance parait devoir ètre considėrable. 


\section{CIIAPITRE V.}

LE THE்ORÈME DE M. PICARD.

\section{Le théorème de M. Picard.}

Nous avons déjà parlé (p.: 5 et 6 ) d'un théorème important découvert par M. Picard en 1880 ; ce théorème consiste en ce que une fonction entière $\mathrm{F}(z)$ telle que les équations

$$
\begin{aligned}
& \mathbf{F}(z)=a, \\
& \mathbf{F}(z)=b, \quad a \neq b
\end{aligned}
$$

n'aient pas de racines, se réduit nécessairement à une constante. Nous donnerons dans la Note I, une démonstration génćrale de ce théorème, indépendante de la théorie des fonctions nodulaires.

Dans ce Chapitre, nous allons, comme nous l'avons déjà fait, nous restreindre aux fonctions de genre fini; il est alors aisé de généraliser le résultat de M. Picard, de sorte que nous gagnerons en précision ce que nous perdrons en étendue ( ${ }^{1}$ ).

En même temps que le théorème que nous venons de rappeler, M. Picard a démontré, toujours à l'aide de la théorie des fonctions modulaires, le théorème plus général que voici : $S i$ les iquations

$$
\begin{aligned}
& \mathbf{F}(\boldsymbol{z})=a, \\
& \mathbf{F}(\boldsymbol{z})=b, \quad a \neq b
\end{aligned}
$$

ont chacune un nombre limité de racines, la fonction entière $\mathrm{F}(z)$ se réduit à un polynome. Nous allons, en supposant $\mathrm{F}(z)$

(1) Les généralisations que nous indiquerons peuvent, d'ailleurs, être étendues aux fonctions entières les plus générales, comme je l'ai montré dans mon Mémoire des Acta malhematica déjà eité. 
de genrefini, démontrer une proposition un peu plus étendue; nous considérerons les équations

$$
\left\{\begin{array}{l}
\mathrm{F}(z)=\mathrm{P}(z), \\
\mathrm{F}(z)=\mathrm{Q}(z),
\end{array}\right.
$$

$\mathrm{P}(z)$ et $\mathrm{Q}(z)$ étant deux polynomes différents; nous montrerons que si chacune de ces équations a un nombre limité de racines, $\mathrm{F}(z)$ est un polynome $\left({ }^{1}\right)$.

En effet, si chacune des équations (1) a un nombre limité de racines on a, évidemment,

$$
\left\{\begin{array}{l}
\mathrm{F}(z)-\mathrm{P}(z)=\mathrm{A}(z) e^{\mathbf{I}(z)}, \\
\mathrm{F}(z)-\mathrm{Q}(z)=\mathrm{B}(z) e^{\mathbf{k}(z)},
\end{array}\right.
$$

$\mathrm{A}(z)$ et $\mathrm{B}(z)$ étant des polynomes.

D'autre part, la fonction $F(z)$ étant de genre fini, il résulte manifestement de remarques précédemment faites que $\mathrm{H}(z) \mathrm{et}$ $\mathrm{K}(z)$ sont des polynomes; dès lors, en retranchant membre à membre les égalités (2), on obtient l'identité

$$
\mathrm{Q}(z)-\mathrm{P}(z)=\mathbf{\Lambda}(z) e^{\mathbf{H}(z)}-\mathrm{B}(z) e^{\mathbf{k}(z)},
$$

dans laquelle $\mathrm{P}, \mathrm{Q}, \mathrm{A}, \mathrm{B}, \mathrm{H}, \mathrm{K}$ sont des polynomes. On sait que $P$ étant différent de $Q$, cette identité exige que $H$ et $\mathrm{K}$ soient des constantes; $F(z)$ est donc bien un polynome, ce qu'il fallait é!ab!ir.

Observons, avec M. Hadamard, que ce mode de démonstration suggère immédiatement de nombreuses généralisations. Par exemple si $F(z)$ et $G(z)$ sont des fonctions de genre fini ayant un nomlore limité de zéros, l'équation

$$
\mathrm{P}(z) \mathrm{F}(z)+\mathrm{Q}(z) \mathrm{G}(z)=\mathrm{R}(z)
$$

dans laquelle $\mathrm{P}, \mathrm{Q}, \mathrm{R}$ sont des polynomes quelconques, a nicessairement une infinité de racines, à moins que $\mathrm{R}(z)$ étant $\mathrm{nul}$, le quolient $\frac{\mathrm{F}(z)}{\mathrm{G}(z)}$ ne se réduise à une fraction rationnelle. On énoncerait aisément des généralisations analogues.

A un autre point de vue, on peut étendre une partie des résultats précédents à des fonctions dont le genre n'est pas fini; nous

\footnotetext{
(1) Voyez Hadarard, Mémoire cité, $§ 15,16,17$.
} 
allons développer ce point avec quelques détails, bien que, par cette méthode, l'on aille ainsi moins loin que nous ne le ferons dans la Note I. Mais le procédé de démonstration que nous emploierons, après M. Hadamard, est intéressant en lui-mème et nous fournira l'occasion de faire, chemin faisant, des remarques assez importanles.

Il s'agira simplement du premier théorème de M. Picard, c'està-dire de la proposition d'après laquelle il ne peut pas exister de fonction entière non constante $\mathrm{F}(z)$ telle que les équations

$$
\begin{aligned}
& \mathrm{F}(z)=a, \\
& \mathrm{~F}(\bar{z})=b, \quad a \neq b
\end{aligned}
$$

l'aient aucune racine.

S'il existc une fonction $\mathrm{F}(z)$ ayant cette propriété, on peut écrire

$$
\mathrm{F}(z)-a=e^{\mathbf{G}(z)},
$$

$\mathrm{G}(z)$ étant une fonction entière; l'équation

devient alors

$$
\mathrm{F}(z)=b
$$

$$
e^{(\mathbf{i}(z)}=b-a \text {. }
$$

Par hypothèse, cette équation n'a pas de racine; or, pour que z vérifie cette équation, il suffit que l'on ait

$$
\mathrm{G}(z)=\log (b-\ldots a)+2 k i \pi,
$$

$l i$ étant un entier quclconque [on a pris pour $\log (b-a)$ une détermination arbitraire; on se rappelle que $b-a$ est cssentiellement différent de zéro]. Donc, l'hypothèse faite sur $F(z)$ a pour conséquence que l'équation (2) n'a, quel que soit $k$, aucune racine; en particulier, il existe deux nombres différents $a^{\prime}$ et $b^{\prime}$ tels que les équations

$$
\begin{aligned}
& \mathrm{G}(z)=a^{\prime}, \\
& \mathrm{G}(z)=b^{\prime}
\end{aligned}
$$

n'aient pas de racines. Ainsi la démonstration du théorème de M. Picard pour la fonction $\mathrm{F}(z)$ se ramène à la démonstration du même théorène pour la fonction $\mathrm{G}(z)$. Il semble que l'on n'ait fail que déplacer la difficulté, mais qu'elle subsiste aussi grande. Cependant, dans des cas très étendus, on pourra s'arranger de ma- 
nière à être ramené au cas où $\mathrm{G}(z)$ est de genre fini, cas pour lequel le théorème vient d'être démontré.

Supposons qu'il existe un nombre $m$, tel que, $M_{1}(r)$ désignant le maximum du module de $\mathrm{F}(z)$ pour $|z|=r$, l'on ait

$$
\mathrm{M}_{1}(r)<e^{e^{r^{m}}} \text {. }
$$

Récrivons l'identité

$$
\mathbf{F}(z)-a=e^{\mathbf{C}(z)} ;
$$

on a donc, pour toute valeur de $z$,

$$
\left|e^{\mathrm{C}(\Leftrightarrow)}\right|<e^{e^{r^{m}}} .
$$

Désignons par $A(r)$ le maximum des valeurs positives de la partie réclle de $\mathrm{G}(z)$ pour $|z|=r$; il cxiste unc valeur de $z$, de module $r$, pour laquelle ce maximum est atteint ( 1 ); pour cette valeur de $z$ on a

$$
\left|e^{\mathbf{f}(z)}\right|=e^{\cdot(r)}
$$

on peut donc écrire, pour toute valeur de $r$,

c'est-à-dire

$$
e^{\mathbf{1}(r)}<e^{e^{r^{m}}}
$$

$$
\mathbf{A}(r)<e^{r^{m}}
$$

Dìs lors, on sait que $\mathrm{G}(\xi)$ est du genre fini. D'une manière plus précise (page 65), si l'on désigne par $M(r)$ le maximum du module de $G(z)$ pour $|z|=r$, et par $\varepsilon$ un nombre positif arbitrairement petit, on a, pourvu que $r$ soit assez grand,

$$
\mathrm{I}(r)<e^{r^{m+\varepsilon}}
$$

Ainsi le premier théorème de M. Picard se trouve démontré pour les fonctions $F(z)$ vérifiant l'inégalité

$$
|\mathrm{F}(z)|<e^{e^{r^{m}}} \text {. }
$$

Supposons maintenant que la fonction $\mathrm{F}(z)$ vérifie l'inégalite,

$$
|\mathrm{F}(z)|<e^{e^{r^{m}}}
$$

si l'on pose encore

$$
\mathbf{F}(z)-a=e^{\mathbf{G}(z)}
$$

( $\left.{ }^{1}\right)$ On sait, en effet, qu'une fonction harnonique régulière dans un contour fermé attci.lt son maximum en un point du contour. 
on aura, en conservant les mêmes notations,

$$
\mathrm{A}(r)<e^{e^{r^{m}}} .
$$

Or, nous avons vu que l'on a

$$
\mathbf{M}(r)<-\frac{k r}{\varepsilon} \Lambda(r+\varepsilon)
$$

$k$ étant une constante indépendante de $r$, pourvu que $r$ soit assez grand. On en conclut (1) que, quelque petit que soit $\eta$, on aura pour $r$ assez grand

$$
\mathbf{M}(r)<e^{e^{r^{m}}},
$$

c'cst-à-dire que la fonction entière $\mathrm{G}(z)$ rentre dans la catégoric de celles pour lesquelles le théorème de M. Picard est démontré; il en est dès lors de même de la fonction $\mathrm{F}(z)$.

En raisonnant de la même manière, on arrivera à démontrer de proche en proche le théorème pour toutes les fonctions $\mathbf{F}(z)$ qai vérifient une inégalité de la forme

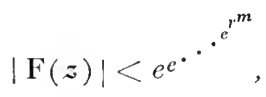

le nombre des exposants superposés étant quelconque. On ne restreint d'ailleurs pas la généralité en supposant $m=1$, puisque l'on peut augmenter d'une unité le nombre des exposants.

Malheureusement, on n'épuise pas ainsi l'ensemble des fonclions entières. Si l'on pose

$$
\begin{aligned}
& \varphi_{1}(z)=e^{z}, \\
& \varphi_{2}(z)=e^{c^{z}}=e^{\eta_{1}(z),} \\
& \varphi_{3}(z)=e^{\varphi_{2}(z)}, \\
& \ldots \ldots \ldots \ldots \ldots, \\
& \varphi_{m}(z)=e^{\eta_{m-1}(z)}, \\
& \ldots \ldots \ldots \ldots \ldots,
\end{aligned}
$$

il est clair que la série

$$
F(z)=\sum \frac{\varphi_{m}(z)}{\varphi_{m}(m)}
$$

représente une fonction entière; si l'on désigne par M $(r)$ le maxi-

(') Il suffil, par exemple, de prendre $\varepsilon=\mathrm{I}$. 
mum du module de $\mathrm{F}(z)$ pour $|z|=r$, on a visiblement, quel que soit $m$, pourvu que $r$ soit assez grand (1),

$$
\mathrm{M}(r)>\varphi_{m}(r) \text {. }
$$

Nous avons tenı à détailler cette démonstration parce qu'on pourrait ètre conduit à regarder comme évident que, si la fonction $e^{\mathbf{G}(z)}$ vèrifie, quel que soit $z$, l'inégalité

$$
\mid e^{\mathrm{G} \mid \Leftrightarrow)}<e^{g(n)} \text {. }
$$

l'on a atıssi, quel que soit $z$,

$$
|\mathrm{G}(z)|<0(r)
$$

Or, cela n'est pas exact en général; on a simplement

$$
\mathbf{A}(r)<0(r),
$$

et il faut utiliser les développements des pages 6j-68 pour trouver une relation d'inégalité entre $\mathbf{M}(\boldsymbol{r})$ et $\theta(\boldsymbol{r})$.

Celle relation s'obtient aisément, comuse nous l'avons vi:, lorsque $\theta(r)$ vérifie, pour quelque valeur de $m$, l'inégalité

$$
\theta(r)<\rho_{m}(r)
$$

mais, dans le cas où $\theta(r)$ ne vérifierait celte inégalité pour aucure valeur de $m$, il faudrait reprendre l'inégalité

$$
\mathbf{M}(r)<\frac{k \Xi}{r} \mathrm{~A}(r+\xi)
$$

el la traiter par des méthodes analogues à celles de la Note I. D'ailleurs, ces remarques n'ont pas de rapport avec la démonstralion du théorème de M. Picard, laquelle ne scmble pas, par celte méthode, pouvoir être étendue plus que nous ne venons de le faire avec M. Hadamard.

( $\left.{ }^{1}\right)$ On pourrait rattacher ce résultat au théorème fondamental de Paul du BoisReymond sur les fonctions positives croissantes (voir mes Leçons sur la Théorie des fonctions, Note I). On utiliserait pour cela une remarque de M. Poincaré, d'après laquelle, ètant dounée une fonction positive croissante, on peut trouver une fonction entière croissant plus vite (American Journal of Mathematics, t. XIV, p. 214). 
Le théorème de M. Picard généralisé.

Nous allons montrer maintenant comment une généralisation aisée du théorème de M. Picard permet de fournir une réponse à la question suivante : $\mathrm{F}(z)$ étant une fonction entière, que peut-on dire de général sur la distribution dans ce plan des racines

$$
\mathrm{F}(z)=a,
$$

$a$ étant une constante quelconque? Le nombre des racines de cette équation, dont le module est inférieur à $r$, est une fonction de $r$. Le théorème de M. Picard nous apprend que, F(z) étant donné, cette fonction $\varphi_{a}(r)$ croît indéfiniment avec $r$, sauf peut-être pour une valeur de $a$. La généralisation dont nous allons parler nous renseignera d'une manière plus précise sur cette fonction; le point important sera toujours l'existence possible d'un cas d'exception unique.

Nous nous bornerons, comme nous l'avons fait jusqu'ici, aux fonctions de genre fini; nous dirons seulement, à la fin du parazraphe, quelques mots d'un cas un peu plus général.

Considérons une fonction $\mathbf{F}(z)$ de genre fini et supposons d'abord que son ordre apparent ne soit pas un nombre entier; nous savons alors que l'ordre réel est égal à l'ordre apparent; nous connaissons donc l'exposant de convergence de la suite de ses zéros; cet exposant reste le même pour toutes les fonctions $\mathrm{F}(z)-a$, lesquelles ont visiblement le même ordre apparent. Ici le cas d'exception de M. Picard ne peut pas se présenter: quand l'on se borne aux fonctions de genre fini, il est clair que le cas que nous venons d'examiner est celui que l'on devrait considérer comme le plus gẻnéral; à ce point de vue, l'étude des zéros paraît ne se rattacher qu'indirectement au théorème de M. Picard, puisque, dans la plupart des cas, on peut affirmer a priori que le cas d'exception est impossible. Mais sans faire la théorie des fonetions de genre infini, nous pouvons indiquer que, pour toutes ces fonctions, le cas d'exeeption de M. licard est possible a priori, c'est-à-dire lorsqu'on connaît seulement le mode de croissance (ce qu'on peut encore appeler l'ordre apparent, qui seulement est 
maintenant un nombre infini); par suite, le cas que nous allons maintenant examiner (ordre apparent entier), bien que très parliculier dans la théorie des fonctions de genre fini, est, en fait, le cas le plus général lorsque l'on considère les fonctions entières dans leur ensemble ( 1 ).

Soit donc maintenant $F(z)$ une fonclion entière dont l'ordre apparent esı un nombre fini $p$. Soient $\varphi_{(}(z)$ et $\varphi_{1}(z)$ des fonctions cntières quelconques d'ordre apparent inférieur à $p$. Nous allons montrer que parmi l'ensemble des fonctions

$$
p(z) F(z)-p_{1}(z)
$$

il y en a une au plus dont l'ordre réel est inférieur à $p\left(^{2}\right)$. En d'autres termes, les équations de la forme

$$
F(z)=\frac{P_{1}(z)}{\varphi(z)}
$$

sont toutes, sauf une au plus, telles que l'exposant de convergence de la suite de leurs racines est égal à $p$.

Supposons en effet que les deux fonctions

$$
\begin{aligned}
& \varphi(z) \mathrm{F}(z)-\varphi_{1}(z), \\
& \bigsqcup(z) \mathrm{F}(z)-\varphi_{1}(z)
\end{aligned}
$$

aient un ordre apparent inférieur à $p$; on aura

$$
\begin{aligned}
& \varphi(z) \mathrm{F}(z)-\varphi_{1}(z)=\Phi(z) e^{\mathbf{P}(z)}, \\
& \varphi(z) \mathbf{F}(z)-\psi_{1}(z)=\Psi(z) e^{Q(z)},
\end{aligned}
$$

$\Phi(\xi)$ et $\Psi(\xi)$ étant des fonctions entières d'ordre apparent inférieur à $p, \mathrm{P}(z)$ et $\mathrm{Q}(z)$ étant des polynomes de degrép. En éliminant $\mathrm{F}(z)$ entre ces équations, on obtient

$$
\psi_{1}(z) \varphi(z)-\varphi_{1}(z) \psi(z)=\psi_{1}(z) \Phi(z) e^{\mathbf{p}_{(z)}}-\varphi_{1}(z) \Psi(z) e^{: !(z)}
$$

( $\left.{ }^{1}\right)$ Bien entendu, les fonctions entières pour lesqueltes le cas d'exception de M. Picard se présente effectivement sont très particulières; ce que nous voulons dire, c'est que, étant donné un mode de croissance (au sens précisé dans un Mémoire déjà cité, p. $37^{2}$ ), il y a des fonctions entières ayant ce mode de croissance et pour lesquelles ce cas d'exception se présente (sauf dans le cas particulier que nous venons d'examiner).

( $\left.{ }^{2}\right)$ D'une manière plus précise, s'il y en a plusicurs, on peut les déduirc de l'uoe d'entre elles en la multipliant par une fonction entière. 
c'est-à-dire une relation de la forme

$$
\mathbf{M}(\boldsymbol{z}) e^{\mathbf{P}(z)}+\mathbf{N}(\boldsymbol{z}) e^{\mathbf{Q}(z)}=\mathrm{L}(z)
$$

dans laquelle $P^{\prime}(z)$ et $\mathrm{Q}(z)$ sont des polynomes de degré effectivcmert égal à $p$, tandis que $\mathbf{M}(z), \mathbf{N}(z), \mathrm{L}(z)$ sont des fonctions entières dont l'ordre apparent est inférieur à $p$. Une telle relation est impossible. En effet, si nous prenons la dérivée des deux membres de l'identité (I), nous obtenons, en désignant les dérivées par des accents,

$$
\left(\mathbf{M}^{\prime}+\mathbf{M} \mathbf{P}^{\prime}\right) e^{\mathbf{P}(*)}+\left(\mathbf{N}^{\prime}+\mathbf{N Q}^{\prime}\right) e^{\mathbf{Q}(z)}=\mathbf{L}^{\prime} .
$$

Les équations (1) el (2) donnent immédiatement

$$
\begin{aligned}
& \left(\mathbf{M N}^{\prime}-\mathbf{N M}^{\prime}\right) e^{\mathbf{P}(z)}=\mathbf{L}\left(\mathbf{N}^{\prime}+\mathbf{N Q}^{\prime}\right)-\mathbf{L}^{\prime} \mathbf{N}, \\
& \left(\mathbf{M N ^ { \prime }}-\mathbf{N} \mathbf{M}^{\prime}\right) e^{\mathbf{Q}(\xi)}=-\mathbf{L}\left(\mathbf{M}^{\prime}+\mathbf{M P}^{\prime}\right)+\mathbf{L}^{\prime} \mathbf{M},
\end{aligned}
$$

c'est-à-dire que l'on a

$$
e^{\mathbf{P}(z)}=\frac{\alpha(z)}{\gamma(z)}, \quad e^{Q(z)}=\frac{\beta(z)}{\gamma(z)},
$$

$\alpha(z), \beta(z), \gamma(z)$ étant des fonctions entières dont l'ordre est inférieur à $p$; nous savons que cela est impossible car, lorsque le quotient de deux telles fonctions est une fonction entière, l'ordre de cette fonction est inférieur à $p$.

Nous avons supposé implicitement que l'on a

$$
M N^{\prime}-N M^{\prime} \neq 0,
$$

c'est-à-dire que le rapport $\frac{M}{N}$ n'est pas une constante; si lion a

$$
\mathbf{M}(z)=\mathrm{CN}(z)
$$

l'équation (1) devient

$$
\mathrm{C} e^{\mathbf{P}(z)}+e^{Q(z)}=\frac{1(z)}{\Lambda(z)}
$$

le second membre est comme le premier une fonction entière; son ordre est donc infériemr à $p$; l'ordre du premièr membre est visiblement égal à $p$, à moins que ce premier membre ne soit identiquement nul, auquel cas on arriverait aisément à la relation

$$
\frac{\varphi_{1}(z)}{\varphi(z)}=\frac{\bigcup_{1}(z)}{\bigcup_{(j)}(z)}
$$


il existe alors deux fonctions entières $\chi_{(z)}$ et $\chi_{1}(z)$ telles que l'on ait

$$
\begin{aligned}
& \varphi_{1}(z)=\theta(z) \%_{1}(z), \\
& \varphi(z)=0(z) \psi_{(z),} \\
& \bigcup_{1}(z)=r_{1}(z) \chi_{1}(z), \\
& \varphi_{(z)}=r_{1}(z) \psi_{(z),}
\end{aligned}
$$

$\theta(z)$ et $\eta(z)$ étant des fonetions entières.

Ainsi, parmi les équations de la forme

$$
\mathrm{F}(z)=\frac{\varphi_{1}(z)}{i(z)},
$$

une au plus est telle que l'exposant de convergence de la suite de ses racines est inférieur à $p$. Il n'est pas inutile de remarquer que, lorsqu'on est dans lc cas d'exception, l'exposant de convergence peut avoir une valeur quelconque inférieure à $p$ : on ne sait rien sur son compte. Il suffit, en effet, de prendre

$$
\mathrm{F}(z)=\mathrm{G}(z) e^{Q(z)},
$$

$Q(z)$ étant un polynome de degré $p$ et $\mathrm{G}(z)$ un produit canonique de facteurs primaires dont l'ordre $\rho$ est inférieur à $p$. Nous laisserons donc complètement de côté le cas d'exception; supposant que nous sommes dans le cas général, nous allons chereher à préciser le plus possible ee que nous savons sur la distribution des racines d'une fonction entière dont l'ordre apparent est donné.

11 nous suffira, pour cela, de nous reporter à des remarques faites plus haut, relativement à l'exposant de convergence d'une suite. Nous savons que, si l'exposant de convergence de la suite

$$
r_{1}, r_{2}, \ldots, r_{n}, \ldots
$$

est égal à $\alpha$, on peut affirmer que l'inégalité

$$
r_{n}>n^{\frac{1}{\hat{\beta}^{--\varepsilon}}}
$$

est vérifiée quelque petit que soit $\varepsilon$ donné d'avance, à partir d'une certaine valeur de $n$. D'autre part, l'inégalité

$$
r_{n}<n^{1+\varepsilon}
$$

est vérifiée pour une infinité de valeurs de $n$.

B. 
Mais il peut arriver qu'il existe aussi une infinité de valeurs de $n$ pour lesquelles cette dernière inégalité n'ait pas lieu.

En d'autres termes, si l'on considère la suite

$$
\frac{\log r_{2}}{\log 2}, \frac{\log r_{3}}{\log 3}, \ldots, \frac{\log r_{n}}{\log n}, \ldots,
$$

on peut affirmer que $\frac{\mathrm{I}}{\lambda}$ est la plus petite de ses limites; mais on n'est pas certain que la suite tende vers la limile unique $\frac{\mathbf{I}}{\lambda}$. Dans la Note II, nous indiquerons comment l'on peut, dans certains cas, montrer qu'il en est bien ainsi, ce qui eomplétera les résultats déjà acquis.

Il est une autre direction dans laquelle on pourrait chercher à préciser nos résultats; les inégalités (1) et (2), même si elles étaient vérifiées pour toute valeur de $n$, sont loin de définir complètement la manière dont croît $r_{n}$; on peut dire que la croissance de $r_{n}$ se trouve renfermée entre eertaines limites, mais on pourrait se proposer de resserrer davantage ees limites. Par exemple, il est manifeste que si l'on prend

$$
r_{n}=n^{\frac{1}{\lambda}}(\log n)^{k}
$$

$k$ étant un nombre positif ou négatif quelconque, les inégalités (I) et (2) sont, quelque petit que soit $\varepsilon$, vérifiées toutes deux à partir de quelque valeur de $n$; ne pourrait-on pas, connaissant le mode de croissance de la fonction entière, remplacer les inégalités ( 2 ) par des inégalités de la forme

$$
\begin{aligned}
& r_{n}>n^{\frac{1}{\rho}}(\log n)^{k-\varepsilon,}, \\
& r_{n}<n^{\frac{1}{\rho}}(\log n)^{k+\varepsilon},
\end{aligned}
$$

dans lesquelles $k$ aurait une valeur connue? Il est clair que l'on serait ainsi mieux renseigné sur la croissanee de $r_{n}$, bien qu'une infinité de fonctions croissantes puissent être placées encore cntre les nouvelles limites; on pourrait d'ailleurs se proposer de les resserrer encore, et ainsi de suite indéfiniment, et même transfiniment ( 1 ).

( ${ }^{1}$ Voir mes Lecons sur la théorie des fonctions, Note II. 
Il est manifeste que pour calculer $r_{n}$ avec une précision déterminée, il est nécessaire de connaître $\mathbf{M}(r)$ avec une précision comparable; nous allons en conclurc que l'on ne peut même pas espérer accomplir le premier des progrès que nous venons de signaler, à moins de recourir à des considérations d'une tout autre nature que celles qui ont été employées jusqu'ici.

Considérons, en effet, les deux fonctions entières

$$
\sin \frac{\pi z}{2}, \quad \frac{1}{\Gamma(z)} ;
$$

les zéros de la première sont

$$
\text { o, } \quad 2, \quad-2, \quad 4, \quad-4, \quad 6, \quad-6, \quad \ldots
$$

et ceux de la seconde

$$
0,-\mathrm{r},-2,-3,-4,-5,-6, \ldots
$$

Au point de vue auquel nous nous plaçons, $r_{n}$ doit être considéré comme croissant de la même manière, pour les deux fonctions entières considérées. Or, si l'on considère le module maximum $\mathrm{M}(r)$ des deux fonctions entières, il a les deux formes distinctes ( 1 )

$$
e^{k r}, \quad r^{k^{\prime} r}
$$

$k$ et $k^{\prime}$ étant des constantes; les logarithmes de ces expressions sont respectivement proportionnels à

$$
r, \quad r \log r \text {. }
$$

On voit donc que les considérations développées jusqu'ici ne permettent pas d'atteindre une précision assez grande pour distinguer entre elles la croissance de ces deux fonctions: nous devons les regarder comme identiques.

En résulte-t-il que l'on doive renoncer à aborder les sujets de recherches que nous indiquions? Nullement, et l'on aperçoit immédiatcment deux voies dans lesquelles on obtiendrait sans doute des résultats : on pourrait d'abord supposer le nombre $p$ assez petit ou même nul ; il en résulterait des facilités spéciales. D'autre part, et cette seconde voie paraît pouvoir être plus féconde en

( $\left.{ }^{1}\right)$ Nous négligeons pour $\frac{I}{\Gamma(z)}$ un facteur accessoirc sans importance. 
résultats, mais en même temps plus malaisée, on pourrait tenir compte des arguments des zéros, dont il n'a pas été question jusqu'ici. On devrait, par exemple, expliquer la différence que nous venons de signaler entre $\frac{1}{\Gamma(z)}$ et $\sin z$ par le fait que les zéros de la première de ces fonctions ont tous même argument, tandis que pour la seconde les arguments on deux valeurs qui diffèrent de $\pi$.

A un point de vue un peu différent, le problème se poserait comme il suit: que peut-on dire de commun sur les modules et les arguments des racines de toutes les équations de la forme

$$
\mathrm{F}(z)=a
$$

où $\mathrm{F}(z)$ est une fonction entière? Ou bien : on donne $\mathrm{F}(z)$ par sa décomposition en facteurs primaires; peut-on en conclure quelque résultat précis sur la décomposition de $\mathrm{F}(z)-a$ ? Par exemple, désignant par $r_{n}$ le module du $n^{\text {itme }}$ zéro de $\mathrm{F}(z)$, par $\mathrm{R}_{/}$le module du $n^{\text {ième }}$ zéro de $\mathrm{F}(z)-a$, peut-on affirmer que, le cas d'exception de M. Picard étant écarté, le rapport $\frac{r_{n}}{R_{n}}$ tend vers l'unité quand $n$ augmente indéfiniment? Nous nous contentons d'indiquer cette question comme type; on pourrait en imaginer une infinité d'autres; mais il serail préférable de trouver d'abord une méthode pour y répondre.

En terminant, nous allons, comme nous l'avons annoncé, indiquer une généralisation du théorème de M. Picard dans laquelle interviennent, en réalité, des fonctions de genre infini; nous pourrons cependanı donner à l'énoncé une forme telle qu'il n'y figure que des fonctions de genre fini. Rappelons qu'une fonction entière est d'ordre réel fini lorsque ses zéros peuvent être considérés comme ceux d'une fonction de genre fini. Voici maintenant le théorème que nous allons démontrer:

Soit $\mathrm{F}(z)$ une fonction entière vérifiant, pour toute valeur de z, l'inégalité (')

$$
|\mathbf{F}(z)|<e^{e^{r^{m}}}
$$

dans laquelle $m$ est une constante; soient, d'autre part,? (z),

( ${ }^{1}$ ) Cette hypothèse sert seulement à simplifier la démonstration; le théorème reste exact si on la supprime. 
$\vartheta_{1}(z), \quad \varphi_{(z)}, \bigcup_{1}(z)$ des fonctions entières de genre fini telles que l'on ait

$$
0 \psi_{1}-\varphi_{1} \psi \neq 0
$$

si les deux fonctions entières

$$
\begin{aligned}
& \varphi(z) \mathbf{F}(z)-\varphi_{1}(z), \\
& \psi(z) \mathbf{F}(z)-\bigcup_{1}(z)
\end{aligned}
$$

sont d'ordre réel fini, la fonction $\mathrm{F}(z)$ est de genre fini.

En d'autres termes, si $\mathrm{F}(z)$ est de genre infini, il peut exister au plus une équation de la forme

$$
\mathrm{F}(z)=\frac{\varphi_{1}(z)}{i(z)}
$$

telle que la suite de ses racines ait un exposant de convergence fini.

Mais reprenons notre premier énoncé; il résulte des hypothèses faites que l'on a

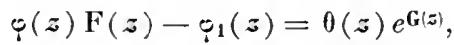

$$
\begin{aligned}
& \psi(z) \mathrm{F}(z)-\psi_{1}(z)=\chi(z) e^{\operatorname{ll}(z)},
\end{aligned}
$$

$\theta(z)$ et $\%(z)$ étant des fonctions de genre fini, tandis que $\mathrm{G}(z)$ et $\mathrm{H}(z)$ sont des fonctions entières ou des polynomes. Pour notre but, il faut prouver que ce sont des polynomes; la fonction $\mathbf{F}(z)$ sera dès lors nécessairement de genre fini.

Or on voit tout d'abord que $\mathrm{G}(z)$ et $H(z)$ ne peuvent être de genre infini; c’est une conséquence de l'inégalité (1); il suffit de raisonner comme à la page $9 \mathbf{r}$. Ce point étant acquis, éliminons $\mathrm{F}(z)$ entre (3) et (4); il vient

$$
\mathbf{M}(z) e^{\mathbf{G}(z)}+\mathbf{N}(z) e^{\mathbf{I l}(z)}=\mathbf{L}(z),
$$

M, N, L étant des fonctions de genre fini dont la dernière est différente de zéro en vertu de (2). Il en résulte tout d'abord que si la différence $\mathrm{G}(z)-\mathrm{H}(z)$ est un polynome $\mathrm{Q}(z)$, on peut affirmer que $\mathrm{G}(z)$ et $\mathrm{H}(z)$ sont des polynomes, car l'identité (5) peut s'écrire

$$
\mathbf{M}(z) e^{\mathbf{Q}(z)}+\mathbf{N}(z)=\mathbf{L}(z) e^{-\mathbf{l}(z)},
$$

le premier membre est de genre fini et, $\mathrm{L}(z)$ étanl de genre fini et différent de zéro, le second membre ne peut être de genre fini que si $H(z)$ est un polynome. 
Différentions maintenant l'identité (5); nous obtenons

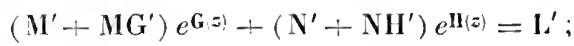

dès lors, en combinant ( 5$)$ et $(6)$, on obtient

$$
\begin{aligned}
& \left(\mathbf{M N}^{\prime}-\mathrm{M}^{\prime} \mathrm{N}\right) e^{\mathrm{G}(\vec{z})}=\mathbf{L}\left(\mathrm{N}^{\prime}+\mathrm{NH}^{\prime}\right)-\mathbf{L}^{\prime} \mathbf{N}, \\
& \left(\mathbf{M} \mathbf{N}^{\prime}-\mathbf{M}^{\prime} \mathbf{N}\right) e^{\mathbf{H}(\tilde{z})}=-\mathbf{L}\left(\mathbf{M}^{\prime}+\mathbf{M G}^{\prime}\right)+\mathbf{L}^{\prime} \mathbf{M} \text {. }
\end{aligned}
$$

Or $M N^{\prime}-M^{\prime} N$ est différent de zéro; sinon le rapport $\frac{M}{N}$ serait constant et cette hypothèse s'écarte aisément (1); dès lors, les

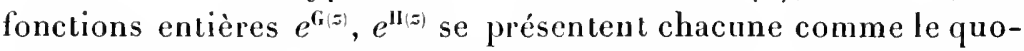
tient de deux fonctions entières de genre fini; elles sont donc elles-mêmes de genre fini, d'après une remarque déjà faite, ce qui établit notre théorème.

Nous en resterons là sur ce sujet; nous espérons avoir montré quelle importance le théorème de $\mathrm{M}$. Picard a dans la théorie des fonctions entières. Sachant qu'il peut exister un cas d'exception, on ne perd pas de temps à chercher un résultat impossible à atteindre: la détermination tout à fait générale de la densité des zéros d'après le mode de croissance; et, d'autre part, sachant que le cas d'exception est unique, on peut espérer obtenir des théorèmes s'appliquant à tous les cas non exceptionnels et ayant, par suite, un champ d'application fortlarge. Nous en avons donné ici quelques-uns, nous irons un peu plus loin dans les Notes; mais nous verrons aussi qu'il y a encore beaucoup à faire dans cette direction et nous signalerons quelques sujets de recherches qui se présentent naturellement et qui ont presque tous leur point de départ dans le théorème qui a fait l'objet de ce Chapitre.

(') En effet, si $\mathbf{I}=\mathrm{CN}$, l'égalité (5) peut s'écrire

$$
e+e^{\mathbf{I}(\tilde{z})-\mathbf{G}(\tilde{z})}=\frac{\mathbf{L}}{\mathbf{N}} e^{-\mathbf{G}(\tilde{z}} ;
$$

d'où, en différentiant,

$$
\left(\mathrm{IJ}^{\prime}-\mathrm{G}^{\prime}\right) e^{\mathrm{Il}-\mathrm{G}}=\left(\frac{\mathrm{L}^{\prime} \mathbf{N}-\mathrm{LN}^{\prime}}{\mathrm{N}^{2}}+\frac{\mathrm{G}^{\prime} \mathrm{L}}{\mathrm{N}^{-}}\right) e^{-\mathrm{G}}
$$

$o r$, s II -- G n'est pas un polynome $\mathrm{H}^{\prime}-\mathrm{G}^{\prime}$ n'est pas nul, et avec cette identité on prouve, comme dans le texte, que $e^{\text {Il }}$ est de genre fini, ce qui suffit. 


\title{
N0TE I.
}

\author{
DÉMONSTRATION ÉLÉMENTAIRE D'UN THÉORÈME DE M. PICARD \\ SUR LES FONGTIONS ENTİ̈ES (1).
}

Je me propose de donner une démonstration directe d'un théorème de 11. Picard sur les fonctions entières, d'après lequel une fonction entière ne devenant égale ni à $a$ ni à $b(a \neq b)$ se réduit nécessairement à une constante. La question se ramène à prouver l'impossibilité d'une relation de la forme

$$
e^{(\boldsymbol{\theta})(z)}+e^{\left(\mathbf{b}_{i}(z)\right.}=1,
$$

$G$ et $G_{1}$ étant des fonctions entières. Nous poserons, $n$ étant un entier positif, nul ou négatif,

$$
\mathrm{G}_{1}(z)-2 n i \pi=e^{\Gamma_{n}(z)} .
$$

Soient $\mathrm{M}(r)$ le maximum de $|\mathrm{G}(z)|$ lorsque $|z| \leqq r, \mathrm{~A}(r)$ la plus grande valeur positive de la partie réelle $\mathrm{P}$ de $\mathrm{G}(z)$ lorsque $|z|=r$ et $-\mathrm{B}(r)$ la plus grande valeur négative de $\mathrm{P}$ pour $|z|=r$. A l'aide de $\mathrm{G}_{1}$ et de $\mathbf{F}_{n}$, nous définirons de mème $\underline{M}_{1}, \mathbf{A}_{1}, \mathbf{B}_{1}, \mu_{n}, x_{n}, \beta_{n}$. Nous désignerons par $\mathbf{K}$ une constante qui ne sera pas la même dans toutes nos inégalités, mais qui sera comprise entre des limites finies, par exemple entre o,oor et rooo; nous remarquerons que $\mathbf{M}, \mathbf{A}, \mathbf{B}, \ldots$ augmentent indéfiniment avec $r$ et nous supposerons $|z|$ assez grand pour qu'ils soient très grands par rapport aux termes coustants de $\mathrm{G}$ et $\mathrm{G}_{1}$. Posons, $a_{0}$ et $a_{0}^{\prime}$ étant réels, ainsi que $P$ et $Q$,

$\mathrm{G}(z)=a_{0}+i a_{0}^{\prime}+a_{1} z+a_{2} z^{2}+\ldots+a_{m} z^{m}+\ldots=\mathrm{P}(r, \theta)+i \mathrm{Q}(r, \theta)$.

(') Je reproduis ici textuellement une Note insérée le I m mai 1896 dans les, Comptes rendus; on y trouvera un exemple de la nature des raisonnements qu'on est amené à employer lorsqu'on ne fait aucune hypothèse sur la croissance des fonctions entières que l'on considère; on ne peut alors, faute d'étalon déterminé, comparer entre elles directement les diverses fonctions positives croissantes qui s'introduisent; on est amené à faire, par un détour, une comparaison indirecte. 
On a manifestement (Cf. Hadamand, Comptes rendus, t. CXIV, p. 1053)

$$
\begin{gathered}
\pi r^{m} a_{m}=\int_{0}^{2 \pi} \mathrm{P}(\theta, r) e^{-i m 0} d \theta, \quad 2 \pi a_{0}=\int_{0}^{2 \pi} \mathrm{P} d \theta ; \\
\pi r^{m}\left|a_{m}\right|<\int_{0}^{2 \pi}|\mathrm{P}| d \theta, \\
\pi r^{m}\left|a_{m}\right|+2 \pi a_{0}<\int_{0}^{2 \pi}(|\mathrm{P}|+\mathrm{P}) d 0<4 \pi \mathrm{A}(r), \\
\pi r^{m}\left|a_{m}\right|-2 \pi a_{0}<\int_{0}^{2 \pi}(|\mathrm{P}|-\mathrm{P}) d \theta<4 \pi \mathrm{B}(r) .
\end{gathered}
$$

Supposons que le module de $z$ soit inférieur ou égal à un nombre $\rho<r$ nous aurons

$$
\mathrm{G}(z)<\left|a_{0}\right|+\left|a_{0}^{\prime}\right|+\left[\left\{\mathrm{A}(r)+2\left|a_{0}\right|\right]\left(\frac{P}{r}+\frac{p^{2}}{r^{2}}+\ldots\right),\right.
$$

et, par suite,

$$
\mathbf{M}(\rho)<\mathbf{K} \frac{\rho \mathbf{A}(r)}{r-\rho}
$$

La mème inégalité a lieu en remplaçant $A$ par $B$ ou en introduisant $\mathbf{A}_{1}$, $B_{1}, M_{1}$. Pour $\alpha_{n}, \beta_{n}, \mu_{n}$, il faut remarquer que dans $\Gamma_{n}$ le terme constant est, lorsque $n$ est grand, de l'ordre de grandeur de $\log |n|$ (nous pouvons supposer que le cofficient de $i$ est compris entre $-\pi$ et $+\pi$ ); on aura done $\left({ }^{1}\right)$

$$
\mu_{n}(\rho)<K_{\rho} \frac{\alpha_{n}(r)+\log |n|}{r-\rho} .
$$

Cela posé, nous pouvons donner à $z$ une valeur $z_{0}$ ayant un module donné $r$ et telle que la partie réelle de $\mathrm{G}(z)$ soit égale à $-\mathrm{B}(r)$; on a alors

$$
\left|e^{\mathbf{G}_{1}\left(\tilde{z}_{0}\right)}-\mathbf{I}\right|=e^{-\mathbf{B}(r)}
$$

et, par suite, $n$ étant un entier déterminé,

$$
\left|\mathrm{G}_{1}\left(z_{0}\right)-2 n i \pi\right|=\mathbf{K} e^{-\mathbf{B}\langle\nu\rangle} .
$$

On a d'ailleurs évidemment, puisque $\left|z_{0}\right|=r$,

$$
|2 n \pi|<\mathbf{M}_{1}(r)+\mathbf{K} e^{-\mathbf{B}(r)}<\mathbf{K} \mathbf{M}_{1}(r) ;
$$

( $\left.{ }^{1}\right)$ Dans celte inégalité el les suivantes, $\operatorname{lor} \mathbf{q} q u e$ est nul, $\log |n|$ doit ètre remplacé par zéro. 


$$
\mathrm{G}_{1}\left(z_{0}\right)-2 n i \pi=e^{\Gamma_{n}\left(z_{0}\right)}
$$

on a donc

et, a fortiori,

$$
\left|\Gamma_{n}\left(z_{0}\right)\right|>\mathrm{KB}(r)
$$

$$
\mu_{n}(r)>\mathrm{KB}(r)
$$

De même, si $R>r$, d'après (2) et (5),

$$
\begin{gathered}
e^{\boldsymbol{x}_{n}(\mathrm{R})}<\mathrm{M}_{\mathrm{I}}(\mathrm{R})+|2 n \pi|<\mathrm{KM}_{\mathbf{1}}(\mathrm{R}), \\
\boldsymbol{\alpha}_{n}(\mathrm{R})+\log |n|<\mathrm{K} \log \mathbf{M}_{\mathbf{1}}(\mathrm{R}) .
\end{gathered}
$$

En remplaçant dans (4) $r$ et $\rho$ par $R$ et $r$, tenant compte de $(z)$ et $(8)$, on a

$$
\mathrm{B}(r)<\mathrm{K} \mu_{n}(r)<\mathrm{KR} \frac{\log \mathbf{M}_{1}(\mathrm{R})}{\mathrm{R}-r} .
$$

Or, $p$ étant inférieur à $r$, on a, d'après (4),

$$
\mathbf{A}(\rho)<\mathbf{M}(\rho)<\mathbf{K} \frac{\rho \mathbf{B}(r)}{r-?} .
$$

D'autre part, d'après (1), on a

$$
\mathbf{A}_{1}(\rho)<\mathbf{K A}(\rho)
$$

donc, si $\rho^{\prime}<p$, on a

$$
\begin{aligned}
& M_{1}\left(p^{\prime}\right)<K p^{\prime} \frac{A_{1}(p)}{p-p^{\prime}}<K p^{\prime} \frac{A(p)}{p-p^{\prime}} \\
& <\mathrm{K} \frac{\rho \rho^{\prime} \mathrm{B}(r)}{(r-\rho)\left(\rho-\rho^{\prime}\right)}<\mathrm{K} \frac{\mathrm{R}_{\rho} \rho^{\prime} \log \mathrm{M}_{\mathbf{1}}(\mathrm{R})}{(\mathrm{R}-r)(r-\rho)\left(\rho-\rho^{\prime}\right)} .
\end{aligned}
$$

En supposant $R-r=r-p=\rho-\rho^{\prime}$, on en conclut

$$
M_{1}(R)>K \frac{\left(R-\rho^{\prime}\right)^{6}}{R^{6}}\left[M_{1}\left(\rho^{\prime}\right)\right]^{2} .
$$

Il suffit de faire successivement

$$
\begin{gathered}
\rho^{\prime}=\lambda, \quad \mathrm{R}=\lambda+h ; \quad \rho^{\prime}=\lambda+h, \quad \mathrm{R}=\lambda+h+\frac{h}{2} ; \\
\rho^{\prime}=\lambda+h+\frac{h}{2}, \quad \mathrm{R}=\lambda+h+\frac{h}{2}+\frac{h}{4} ; \quad \ldots,
\end{gathered}
$$

pour constater que si $M_{1}(\lambda)$ est assez grand et $h$ convenablement choisi, $\mathbf{M}_{1}(\lambda+2 h)$ dépasse toute quantité assignable; le rayon de convergence de $\mathrm{G}_{1}(z)$ serait donc au plus égal à $\lambda+2 h$.

Je termine en énonçant la proposition suivante qui, pour moi, n'est pas douteuse, bien que je ne l'aie pas démontrée rigoureusement en général : 
106 Note 1. - démonstration Élémentaire d'Un théonème de m. PiCard.

$G(z)$ étant une fonction entière, M. Hadamard a indiqué une limite supérieure équations $\mathrm{G}(z)=\mathrm{P}(z)$, dans lesquelles $\mathrm{P}(z)$, est un polynome, il $y$ en au plus une telle que le nombre de ses racines de module inférieur. à $r$ soit, pour $r$ très grand, inférieur à $\log \varphi(r)\left({ }^{1}\right)$.

( $\left.{ }^{1}\right)$ Dans mon Mémoire déjà cité des Acta mathematica (t. XX), publié après la Note qui est reproduite ici, j’ai démontré une proposition encore plus précise que celle-là (voir Comptes rendus, 12 octobre 18,6 ). 


\section{NOTE II.}

LES FONGTIONS A GROISSANCE RÉGULIÉRE.

Définitions et énoncés.

Considérons une fonction entière $\mathbf{F}(z)$ d'ordre fini et différent de zéro: soit $M(r)$ Ie maximum de son module pour $|z|=r$; il existe certainement deux nombres $x$ et $\beta$ tels que l'on ait, pour toute valeur de $r$,

l'expression

$$
e^{r^{\alpha}}<\mathbf{M}(r)<e^{r^{3}}
$$

$$
\frac{\log \log M(r)}{\log r^{r}}
$$

est donc toujours comprise entre $\alpha$ et $\beta$; dès lors, elle tend nécessal-rement vers une ou plusieurs llimites remplissant un intervalle compris entre $\alpha$ et $\beta$ (et pouvant coüncider avec l'intervalle $\alpha \beta$ ). Nous dirons que la fonction $\mathrm{M}(r)$ est à croissance régulière si cet intervalle se réduit à un point, c'est-à-dire si l'expression (1) tend vers une limite p lorsque $r$ augmente indéfiniment.

Dans ce cas, la fonction entière $\mathbf{F}(\vec{z})$ sera dite elle-même à croissance régulière.

La définition que nous venons de donner se rattache intimement aux considérations sur les ordres d'infinitude, développées à la fin du Chapitre I (p. 22 et 23); en se servant de ces définitions on voit que $\mathrm{M}(r)$ est à croissance régulière dans le cas où l'ordre d'infinitude de $\log \mathrm{W}(r)$ est déterminé ( $\left.{ }^{1}\right)$.

Nous désignerons, comme nous l'avons fait jusqu'ici, par $a_{n}$ les zéros de $\mathrm{F}(z)$ et par $r_{n}$ leurs modules; d'après la définition qui vient d'ètre rappelée, l'ordre d'infinitude de $r_{n}$ est déterminé s’il existe un nombre $\lambda$ tel que,

(1) On pourrait convenir de parter aussi de P'ordre d'infinitude de $\mathrm{I}(r)$ (voir mon Mémoire sur les séries divergentes. Annales de l'École Normale, 1899), mais nous ne pouvons iei faire une théorie complète des ordres d'infinitude; nous espérons pouvoir y revenir un jour en détail; nous nous contentons de donner, aussi simplement que possible, les quelques définitions qui nous sont nécessaires. 
quelque petit que soit $\approx$ donné d'avance, il existe un nombre $m$ tel que l'inégalité

entraîne les inćgalités

$$
n>m
$$

$$
n^{\lambda-\varepsilon}<r_{n}<n^{\lambda+\varepsilon}
$$

On voit que l'exposant de convergence de la suite des $r_{n}$ est égal à $\frac{\mathbf{I}}{\lambda}$; nous le désignerons par ? et nous supposerons que ce n'est pas un nombre entier. Enfin nous supposerons que $\mathrm{F}(z)$ dont l'ordre réel est ćgal à $\rho$, d'après ce qui précède, a aussi p comme ordre apparent : c'està-dire que l'on ne se trouve pas dans le cas d'exception de $\boldsymbol{M}$. Picard.

Cela posé, nous nous proposons de démontrer dans cette Note les deux théorèmes suivants :

$\mathrm{I}^{\circ}$ Si l'ordre d'in finitude de $r_{n}$ est déterminé, la fonction $\mathbf{F}(z)$ est ¿̀ croissance régulière;

$2^{\circ}$ Si la fonction $\mathrm{F}(z)$ est à croissance régulière, l'ordre d'infinitude de $r_{n}$ est déterminé.

Il n'est pas inutile de préciser ce que ces énoncés ajoutent aux résultats déjà acquis :

$I^{\circ}$ Nous avons démontré que si $\rho$ est l'exposant de convergence de la suite des $r_{n}$, e'est-à-dire, si, quel que soit \& donné à l'avance, il existe un nombre m tel que l'inégalité

$$
n>m
$$

entraine

$$
r_{n}>n^{\frac{1}{\rho}-\varepsilon}
$$

tandis que l'inégalité

$$
r_{n}<n^{\frac{1}{\rho}}+\varepsilon
$$

est vérifiée pour une infinité de valeurs de $n$, il en résulte que l'ordre apparent de $\mathrm{F}(z)$ est égal à $\rho$, c'est-à-dire que, quel que soit à donné à l'avance, il existe un nombre $\mathrm{R}$ tel que l'inégalité

entraine

$$
r>\mathbf{R}
$$

$$
|\mathbf{F}(z)|<e^{r^{p+1 \cdot 8}}
$$

landis que l'inégalité

$$
\mathbf{M}(r)>e^{r^{i-\varepsilon}}
$$

cst vérifiée pour une infinité de valeurs de $r$ 'croissant indéfiniment.

Notre premier théorème consiste en ce que, si l'on suppose l'inégalité (2) vérifiée dans les mêmes conditions que l'inégalité( (I) (c'està-dire pour $n>m$ et non pas seulement ponr une infinité de valeurs 
de $n$ ), l'inégalité( 4) sera vérifiée dans les mêmes conditions que l'inégalité (3) (c'est-à-dire pour $r>\mathrm{R}$ et non plus seulement pour une infinité de valeurs de $r$ ).

$2^{\circ}$ Nous avons démontré aussi la réciproque de la proposition précédente; si l'on suppose l'ordre apparent de $\mathrm{F}(z)$ égal à $p$, c'est-à-dire si les inégalités (3) et (4) sont vérifiées, la première à partir d'une certaine valeur de $r$, la seconde pour une infinité de valeurs de $r$ croissant indéfiniment, il en résulte que l'exposant de convergence de la suite des $r_{n}$ est égal à p, c'est-à-dire que les inégalités (1) et (2) sont vérifiées, la première à partir d'une certaine valeur de $n$, la seconde pour une infinité de valeurs de $n$.

Notre deuxième théorème consiste en ce que, si l'on suppose de plus que l'inégalité (4)est, comme l'inégalité (3), vérifiée à partir d'une certaine valeur de $r$, il en résulte que l'inégalité (2) est, comme l'inégalité (1). vérifiée à partir d'une certaine valeur de $n$ ( $^{1}$ ).

\section{Le premier théorème.}

Occupons-nous d'abord du premier théorème; il consiste en ce que, si l'ordre d'infinitude de $r_{n}$ est déterminé, la fonction $\mathrm{F}(z)$ est à croissance régulière; il suffit donc de prouver que, si $\mathrm{F}(z)$ n'est pas à croissance régulière, l'ordre d'infinitude de $r_{n}$ n'est pas déterminé.

Dire que $F(z)$ n'est pas à croissance régulière, c'est dire qu'il existe un nombre $\sigma<p$ tel que l'on ait, pour une infinité de valeurs de $r$ croissant indéfiniment,

$$
\mathbf{M}(r)<e^{r^{\sigma}}
$$

le nombre $\rho$ est toujours supposé tel que les inégalités

$$
\begin{aligned}
& |\mathbf{F}(z)|<e^{r^{?+\varepsilon}}, \\
& \mathbf{M}(r)>e^{r^{p-\varepsilon}}
\end{aligned}
$$

soient vérifiées, quel que soit $\varepsilon$ donné d'avance, la première à partir d'une ccrtaine valeur de $r$, la seconde pour une infinité de valeurs de $r$ croissant indéfiniment. L'exposant de convergence de la suite des $r_{n}$ est donc égal à $p$, puisqu'on suppose que l'on ne se trouve pas dans le cas d'exception de M. Picard.

Soient $r$ une valeur du module de z pour laquelle l'inćgalité (5) soit vérifiée et $s$ un nombre fixe supérieur à 2 ; déterminons le nombre $n$ par les inégalités

$$
s r_{n} \leqq r<s r_{n+1} .
$$

( $\left.{ }^{1}\right)$ J'ai énoncé pour la première fois ces résultats dans une Note insérée en I 898 aux Comptes rendus ( $\mathrm{t}$. CXXII, p. 321 ); la démonstration est inédite. 
On a (p. 73$)$, la fonction $\mathbf{M}(r)$ étant croissante,

$$
n \log (s-1)<\log \mathbf{M}\left(s r_{n}\right)<\log \mathbf{M}(r)<r^{\sigma}<\left(s r_{n+1}\right)^{\sigma},
$$

c'est-à-dire

$$
r_{n+1}>\frac{[\log (s-1)]^{\frac{1}{\sigma}}}{s} n^{\frac{1}{\sigma}} .
$$

Cette inégalité est vérifiée pour une infinité de valeurs de $n$ croissant indéfiniment. Or, $\varepsilon$ étant un nombre arbitrairement petit donné à l'avance, elle entraîne, pourvu que $n$ soit assez grand,

$$
r_{n+1}>(n+1)^{\frac{1}{\sigma}-\varepsilon} \text {. }
$$

Or, le nombre $\sigma$ est inférieur à $\rho$; on peut donc prendre $\varepsilon$ assez petit pour que l'on ait

$$
\frac{1}{\sigma}-\varepsilon>\frac{1}{\rho}+\varepsilon,
$$

Dès lors l'inégalité ( 7 ), vérifiée pour une infinité de valeurs de $n$, prouve que l'ordre d'infinitude de $r_{n}$ n'est pas déterminé, car, d'après ce qui précède, on a aussi, pour une infinité de valeurs de $n$, l'inégalité dèjà écrite

$$
r_{n}<n^{\frac{1}{\rho}+\varepsilon} .
$$

Notre premier théorème est done démontré.

\section{Le deuxième théorème.}

La démonstration du deuxième est. un peu moins aisée; il consiste, avonsnous dit, en ce que, si la croissance de $\mathrm{F}(z)$ est régulière, l'ordre d'infinitude de $r_{n}$ est déterminé. Il nous suffira donc de prouver que, si l'ordre d'infinitude de $r_{n}$ n'est pas déterminé, la croissance de $\mathrm{F}(z)$ n'est pas régulière.

Nous supposons, comme nous l'avons dit, que le nombre $\rho$ n'est pas entier; soit $p$ sa partie entière; nous avons, par hypothèse,

$$
\mathbf{F}(z)=e^{Q(z)} \prod_{1}^{\infty}\left(\mathrm{I}-\frac{z}{a_{n}}\right) e^{\frac{z}{a_{n}}+\frac{z^{2}}{2 a_{n}^{2}}+\ldots+\frac{z p}{p a_{n}^{p}},}
$$

le degré du polynome $\mathrm{Q}(z)$ étant au plus égal (1) à $p$.

Nous supposons que l'ordre d'infinitude de $r_{n}$ n'est pas déterminé; il existe donc un nombre $\sigma<p$ tel que l'inégalité

$$
r_{n}>n^{\frac{1}{\sigma}}
$$

( $\left.{ }^{1}\right)$ Si ce degré dépassait $p$, on se trouverait dans le cas d'exception de I. Picard, hypothèse qui a été exclue. 
soit vérifiée pour une infinité de valeurs de $n$. D'ailleurs, s étant un nombre arbitrairement petit donné d'avance, l'inégalité

$$
r_{n}>n \frac{1}{p+\varepsilon}
$$

est vérifiée à partir d'une certaine valeur de $n$ et l'inégalité

$$
r_{n}<n \overrightarrow{p^{p-\varepsilon}}
$$

est vérifiée pour une infinité de valeurs de $n$.

Comme on peut remplacer $\sigma$ par un nombre plus grand sans que l'inégalité (8) cesse d'être vérifiée, nous supposerons $\sigma$ et $\varepsilon$ choisis de manière que l'on ait

$$
p<\sigma<p-\varepsilon<p+\varepsilon<p+\mathbf{I},
$$

ces inégalités excluant l'égalité ( $\left.{ }^{1}\right)$. Le nombre $\varepsilon$ étant ainsi fixé, il existera un nombre fixe $m$ tel que l'inégalité (9) soit vérifiée sous la condition

$$
n>m \text {. }
$$

Nous voulons prouver que la fonction $\mathrm{F}(z)$ n'est pas à croissance régulière, nous savons déjà que son maximum $M(r)$ vérifie, à partir d'une certaine valeur de $r$, l'inégalité

$$
\mathbf{I I}(r)<e^{r^{p+\varepsilon}}
$$

et, pour une infinité de valeurs de $r$ croissant indéfiniment, l'inégalité

$$
\mathrm{M}(r)>e^{r^{p-\varepsilon}} \text {. }
$$

Il faut prouver que cette dernière égalıté ne peut pas être, quel que soit $\varepsilon$, vérifiée pour toute valeur assez grande de $r$; en d'autres termes, $i l$ faut faire voir qu'il existe un nombre $\mu$ inférieur à $p$ et tel que l'on ait, pour une infinité de valeurs de $r$ croissant indéfiniment,

$$
\mathbf{M}(r)<e^{r^{\mu}} .
$$

Dans ce but, nous utiliserons les inégalités ( 8 ) et (9) que nous allons récrire, en posant, pour abréger,

$$
p+\varepsilon=\tau .
$$

Nous avons dit que l'inégalité

$$
r_{n}>n^{\frac{1}{\sigma}}
$$

( 1 ) L'indétermination de l'ordre d'infinitude de $r_{n}$ résulte de ce que les inégalités (8) et (10) sont vérifiées toutes deux pour une infinité de valeurs de $n$, $\sigma$ étant inférieur à $\rho-\varepsilon$. 
est vérifiée pour une infinité de valeurs de $n$ et que l'inégalité

$$
r_{n}>n^{\frac{1}{\tau}}
$$

est vérifiée sous la seule condition

$$
n>m \text {. }
$$

On a, d'ailleurs,

$$
p<\sigma<\tau<p+\mathrm{I}
$$

et

$$
\mathrm{F}(z)=e^{\mathbf{Q}(z)} \prod_{1}^{\infty}\left(1-\frac{z}{a_{n}}\right) e^{\frac{z}{a_{n}}+\ldots+\frac{z^{p}}{p a_{n}^{p}}} ;
$$

il faut prouver qu'il existe un nombre, $\mu<\rho$, tel que l'inégalité (I I) soit vérifiée pour une infinité de valeurs de $r$ croissant indéfiniment.

Désignons par $h$ un entier supérieur à $m$ et tel que l'inégalité (8) soit vérifiée pour $n=h$; on a donc

$$
r_{h}>h^{\frac{1}{\sigma}}
$$

Déterminons le nombre $k^{\prime}$ par Ia condition

$$
h^{\frac{1}{\sigma}}=k^{\frac{1}{\tau}}
$$

et soit $k$ la partie entière de $k^{\prime}$, c'est-à-dire supposons que l'on ait

$$
k \leqq k^{\prime}<k+\mathrm{I} .
$$

Le nombre $\tau$ étant supérieur à $\sigma, k^{\prime}$ est donc supérieur à $h$; donc $k$ est au moins égal à $h$; la différence $k-h$ deviendra d'ailleurs de plus en plus grande à mesure que $h$ deviendra plus grand, et l'on sait que l'inégalité (12) est vérifiée pour des valeurs de $h$ croissant indéfiniment.

Nous allons chercher à tirer tout le parti possible de l'inégalité (12) en nous servant à la fois de ce que $r_{n}$ croit avec $n$ et de ce que l'inégalité (9) est vérifiée pour toute valeur de $n$ supérieure à $m$.

Pour $m<n<h$, nous écrirons l'inégalité

$$
r_{n}>n^{\frac{1}{\tau}}
$$

Pour $h \leqq n \leqq k$, l'inégalité (12) donne

$$
r_{n}>\frac{1}{h^{\sigma}} \text {. }
$$

Enfin, pour $n \geqq k+\mathrm{I}$, nous reprendrons l'inégalité

$$
r_{n}>n^{\frac{1}{\tau}}
$$

car, d'après la manière dont le nombre $k$ a été choisi, cette inégalité 
devient alors plus avantageuse que l'inégalité ( $I_{f}$ ). Nous allons chercher à trouver une limite supérieure du module de $\mathrm{F}(z)$ en utilisant les inégalités $(9)^{\prime},(14)$ et $(9)^{\prime \prime}$. Nous écrirons tout d'abord

$$
\mathrm{F}(z)=e^{\mathrm{P}(z)} \mathrm{R}(z) \prod_{n=m+1}^{n=\infty}\left(\mathrm{I}-\frac{z}{a_{n}}\right) e^{\frac{z}{a_{n}}+\ldots+\frac{z^{p}}{p a_{n}^{p}},}
$$

$\mathrm{P}(z)$ étant un polynome de degré $p$ au plus et $\mathrm{R}(z)$ un polynome de degré $m$; le nombre s étant donné à l'avance, nous pouvons prendre $r$ assez grand pour que l'on ait

$$
\left|e^{\mathbf{P}(z)} \mathbf{R}(z)\right|<e^{r^{p+\varepsilon}}
$$

nous pouvons donc faire abstraction de ces facteurs et nous borner à considérer le produit

$$
\mathrm{G}(z)=\prod_{n=m+1}^{n=\infty}\left(\mathrm{I}-\frac{z}{a_{n}}\right) e^{\frac{z}{a_{n}}+\ldots+\frac{z^{p}}{p a_{n}^{p}} .}
$$

Désignons par $s$ un nombre compris entre $\sigma$ et $\tau$ et posons

$$
r=h^{\frac{1}{s}}
$$

nous allons chercher le maximum du module de $\mathrm{G}(z)$ pour $|z|=r$; nous poserons

$$
\mathrm{G}(z)=\Pi_{1} \Pi_{2} \Pi_{3}
$$

avec

$$
\begin{aligned}
& \Pi_{1}=\prod_{m+1}^{h-1}\left(\mathrm{I}-\frac{z}{a_{n}}\right) e^{\frac{z}{u_{n}}+\ldots+\frac{z^{p}}{p a n_{n}^{\prime \prime}},} \\
& \Pi_{2}=\prod_{h}^{k}\left(1-\frac{z}{a_{n}}\right) e^{\frac{z}{a_{n}}+\ldots+\frac{z^{p}}{p a n_{n}^{\prime}},} \\
& \Pi_{3}=\prod_{k}^{\infty}\left(1-\frac{z}{a_{n}}\right) e^{\frac{z}{a_{n}}+\ldots+\frac{z^{p}}{p a_{n}^{\prime \prime}}}
\end{aligned}
$$

Pour trouver une limite supéricure des produits $\Pi_{1}, \Pi_{2}, \Pi_{3}$, nous utiliserons successivement les inégalités $(9)^{\prime},\left(I_{\text {q }}\right)$ et $(9)^{\prime \prime}$.

Occupons-nous d'abord de $\Pi_{1}$; on a, en remarquant que $\mathrm{I}+\frac{r}{r_{n}}$ est inférieur à $e^{\frac{r}{r_{n}}}$,

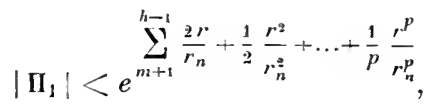

B. 
et, en utilisant $(9)^{\prime}$,

$$
\left|\Pi_{1}\right|<e^{\sum_{m+1}^{h-1} 2 r n n^{-\frac{1}{\tau}}+\frac{1}{2} r^{2} n^{-\frac{2}{\tau}}+\ldots+\frac{1}{p} r^{p} n^{-\frac{p}{\tau}} .}
$$

D'ailleurs

$$
\sum_{m+1}^{h-1} n^{-\frac{i}{\tau}}<\int_{1}^{h} x^{-\frac{i}{\tau}} d x<\frac{1}{1-\frac{i}{\tau}} h^{1-\frac{i}{\tau}}
$$

en remplaçant $h$ par $r$, il vient

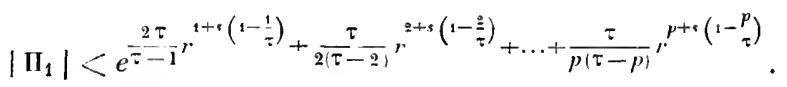

L'exposant le plus élevé de $r$ est visiblement le dernier; désignons-le par $\mu_{1}$, l'on a

$$
\mu_{1}=p+s\left(1-\frac{p}{\tau}\right)=s+p\left(1-\frac{s}{\tau}\right)=\tau-(\tau-p)\left(1-\frac{s}{\tau}\right),
$$

et l'on peut écrire, $\varepsilon$ étant aussi petit que l'on veut, pourvu que $r$ soit assez grand (1),

$$
\left|\mathrm{I}_{1}\right|<e^{r^{r_{1}+\varepsilon}} \text {. }
$$

Passons maintenant à $\mathrm{I}_{2}$; dans ce produit, les $r_{n}$ sont supérieurs à $h^{\frac{1}{\sigma}}$, et comme $r=h^{\frac{1}{s}}$, on peut, quel que soit $s$ supérieur à $\sigma$, supposer $h$ assez grand pour qu'ils soient supérieurs à $2 r$; on a alors

$$
\mathrm{J}_{2}=\prod_{h}^{k}\left(1-\frac{z}{a_{n}}\right) e^{\frac{z}{a_{n}}+\ldots+\frac{z^{p}}{p a_{n}^{p}}}=\prod_{h}^{k} e^{-\frac{z^{p+1}}{(p+1) a_{n}^{p+1}}-\frac{z^{p+2}}{\left(p+2 ; a_{n}^{p+2}\right.}-\cdots},
$$

d'où, en remarquant que $\left|a_{n}\right|>2 \mid z !$

$$
\left|\Pi_{2}\right|<\prod_{h}^{k} e^{\frac{2,^{p+1}}{r_{n}^{p+1}}}
$$

et, en utilisant l'inégalité (I 1 ) et remarquant que le nombre des facteurs est inférieur à $k$,

$$
\left|\Pi_{2}\right|<e^{2 k r^{p+1} / h^{-\frac{p+1}{s}}} \text {. }
$$

( ') Il suffit pour cela de prendre $h$ assez grand. 
On a d'ailleurs $k<k^{\prime}=h^{\frac{\tau}{\sigma}}$ et $h=r^{s}$; il vient donc

$$
\left|\Pi_{2}\right|<e^{r^{u_{2}+\varepsilon}}
$$

en posant

$$
\mu_{2}=\frac{s \tau}{\sigma}+p+1-\frac{p+1}{\sigma} s=\tau-(p+1-\tau)\left(\frac{s}{\sigma}-1\right) .
$$

On obtiendra de mème

$$
\left|\Pi_{3}\right|<e^{2 r^{p+1}} \sum_{k+1}^{\infty} \frac{1}{r_{n}^{q+1}}
$$

or, d'après $(9)^{\prime \prime}$,

$$
\sum_{k+1}^{\infty} \frac{1}{r_{n}^{p+1}}<\sum_{k+1}^{\infty} n^{-\frac{p+1}{\tau}}<\int_{k}^{\infty} x^{-\frac{p+1}{\tau}} d x=\frac{1}{1-\frac{p-1}{\tau}} k^{1-\frac{p+1}{\tau}},
$$

donc

$$
\left|I_{3}\right|<e^{r^{\mu_{3}+\varepsilon}}
$$

en posant ( ${ }^{1}$ )

$$
\mu_{3}=p+1+\left(1-\frac{p+1}{\tau}\right) \frac{s \tau}{\sigma}=\mu_{2} .
$$

Si donc nous désignons par $\mu$ le plus grand des nombres $\mu_{1}$ et $\mu_{2}$, il vient

$$
|\mathrm{F}(z)|<e^{r^{\mu+\varepsilon}}
$$

et quelque petit que soit $\varepsilon$ donné d'avance, cette inégalité est vérifiée pour une infinité de valeurs de $r$ croissant indéfiniment, $z$ pouvant prendre toutes les valeurs de module $r$. Cela revient à dire que l'on a, pour ces valeurs de $r$,

$$
\mathrm{M}(\boldsymbol{r})<e^{r^{\mu+\varepsilon+\varepsilon}}
$$

Il suffit maintenant d'observer que $\mu$ est inférieur à $\tau=p+\varepsilon$ et peut, par suite, e̊tre supposé inférieur à $\rho$ (à condition que $\varepsilon$ ait été pris assez petit), pour que notre proposition soit démontrée : $\mathrm{F}(z)$ n'est pas à croissance régulière.

On peut préciser un peu plus ce résultat en récrivant les valeurs de $\mu_{1}$ et de $\mu_{2} ;$ on a

$$
\begin{aligned}
& \mu_{1}=\tau-(\tau-p)\left(1-\frac{s}{\tau}\right), \\
& \mu_{2}=\tau-(p+1-\tau)\left(\frac{s}{\sigma}+1\right) .
\end{aligned}
$$

(1) Nous remplaçons $k$ par $k^{\prime}=r^{\frac{s \tau}{\sigma}}$; cette approximation est légitime car $k \lesseqgtr k^{\prime}<k+1$ et $k$ est aussi grand que l'on veut. 
Comme l'on a

$$
p<\sigma<s<\tau<p+1
$$

les inégalités excluant l'égalité, les valeurs de $\mu_{1}$ et de $\mu_{2}$ sont bien toutes deux inférieures à $\tau ; \mu$ est donc inférieur à $\tau$.

Mais $\mu_{1}$ et $\mu_{2}$ dépendent du nombre $s$, arbitraire sous la seule condition d'être compris entre $\sigma$ et $\tau$; lorsque $s$ varie, $\mu_{1}$ varie dans le même sens et $\mu_{2}$ en sens inverse; on obtiendra donc la valeur la plus avantageuse de $\mu$ (c'est-à-dire la plus petite possible) en déterminant $s$ de manière que l'on ait $\mu_{1}=\mu_{2}$; la valeur de $s$ obtenue, on calcule celle de $\mu$.

Sans discuter ce résultat, remarquons que si $\tau$ est très yoisin de $p$ ou de $p+I, \mu_{1}$ et $\mu_{2}$ sont respectivement très voisins de $\tau$; d'ailleurs $\mu$ est toujours supérieur à $\sigma\left({ }^{1}\right)$.

\section{Conclusion.}

L'étude complète de la croissance de $\mathbf{M}(r)$, dans le cas où l'ordre d'infinitude de $r_{n}$ n'est pas déterminé, paraît très difficile. La grande difficulté est la suivante: dire que l'ordre d'infinitude de $r_{n}$ n'est pas déterminé c'est dire que le mode de croissance de $r_{n}$ ne peut pas être défini, même à une première approximation, par comparaison avec les fonctions $n^{\lambda}$; il est nécessaire d'introduire de nouvelles fonctions croissantes, qui peuvent être de nature tout à fait arbitraire. Essayer de faire une théorie complète sans préciser la nature de ces nouvelles fonctions, c'est-à-dire en se bornant à la considération des fonctions $n^{\lambda}$, c'est chercher à mesurer une quantité au moyen d'une quantité qui ne serait pas de même nature : cela est manifestement impossible ( ${ }^{2}$ ).

Il faudrait, si l'on voulait étudier le cas où l'ordre d'infinitude de $r_{n}$ n'est pas déterminé, commencer par définir exactement les modes de croissance de $r_{n}$ et étudier en détail chacun de ces modes; ce que l'on pourrait essayer de faire, ce serait de classer ces divers modes en familles que l'on étudierait ensemble $\left({ }^{3}\right)$. Heureusement, les résultats que nous venons d'obtenir, quoique très particuliers en apparence, sont au contraire très

( ${ }^{1}$ ) On peut déduire ces résultats de la formule aisće à établir

$$
\frac{\mu-\sigma}{\tau-\mu}=\frac{\sigma}{p+1-\tau}+\frac{p}{\tau-p} .
$$

(2) Dans la Note suivante, nous parlerons des fonctions à croissance irrégulière, précisément pour bicn montrer quelle est la nature des diffjcultés qui se présentent.

$\left({ }^{3}\right)$ Par exemple, on pourrait chercher à comparer entre elles les fonctions $\mathbf{M}(\boldsymbol{r})$ correspondant à des suites $r_{n}$ dont les modes de croissance seraient comparables entre eux d'une manière simple; il serait sans doute intéressant de développer cette indication. 
généraux au point de vue pratique, car les fonctions entières que l'on rencontre naturellement sont généralement à croissance régulière. Cette importance particulière du mode de croissance exponentiel paraît tenir à des causes très profondes et aura, sans doute, dans l'avenir, des conséquences importantes. J'ai déjà développé cette idée à plusieurs reprises et cherché à la confirmer par des faits; les théorèmes qui viennent d'être démontrés y contribuent poür leur part (') et rapprochent le moment où il sera possible d'exposer la notion de croissance régulière à sa vraie place, au début de la Théorie des fonctions.

Rappelons en terminant que nous avons exclu le cas où le nombre $p$ est entier; il est clair qu'il faut alors tenir compte de la possibilité du cas d'exception de M. Picard. Il est probable que le second théorème prend alors la forme suivante:

Soient $\mathrm{F}(z)$ une fonction à croissance régulière et p son ordre; soient ${ }_{0}(z)$ et $\hat{O}_{1}(z)$ des fonctions entières d'ordre apparent inférieur à $p ; l$ l'on considère les zéros $a_{n}$ de la fonction

$$
\varphi(z) \mathrm{F}(z)+\varphi_{1}(z)
$$

l'ordre d'infinitude de $r_{n}=\left|a_{n}\right|$ est déterminé, sauf au plus pour une valeur particulière du rapport $\frac{\varphi_{1}(z)}{\varphi_{(}(z)}$. D'ailleurs il résulte manifestement des résultats antérieurs que cet ordre d’infinitude, lorsqu'il est déterminé, est égal à $\frac{1}{\rho}$.

Mais il y aurait lieu de démontrer le théorème qui vient d'ètre énoncé; c'est là un sujet de recherches qui peut être abordé avec confiance, car si, contre toute attente, l'énoncé n'est pas exact sous la forme même que nous lui avons donné, il n'est certainement pas besoin de le modifier beaucoup.

Mais, malgré cette lacune, il rèsulte de ce qui précède que, lorsque l'on a une fonction entière d'ordre (ou de genre) fini, la question de savoir si sa croissance est régulière est plus importante que la détermination même de l'ordre : c'est le premier problème que l'on doit aborder, de mème que l'analyse qualitative doit précéder l'analyse quantitative.

Récemment, M. Painlevé a formé des équations différentielles algébriques admettant comme intégrales des fonctions entières nouvelles, c'està-dire ne se réduisant pas à des combinaisons de fonctions connues. Le problème se pose dès lors d'étudier ces fonctions entières, qui vérifient

(') Signalons aussi un résultat fort intéressant, dù à M. E. Lindelöf, au sujet des fonctions réelles définies par une équation différentielle du premier ordre (Bulletin de la Société mathématique de France; 1899). Nous aurons sans doute l'occasion d'y revenir pour en faire ressortir l'importance au point de vue qui nous occupe ici. 
des équations différentielles fort simples. Dans cette étude, la première question à résoudre est la suivante : La croissance de ces fonctions estelle régulière? Il est d'ailleurs très probable qu'elle se résoudra par l'affirmative; dans le cas contraire, on aurait des types de croissance nouveaux, obtenus d'une manière naturelle. Ces types de croissance devraient être étudiés à côté du type exponentiel; il ne serait plus dès lors possible de dire que celui-ci est seul important en Analyse; il resterait cependant le plus important. 


\section{NOTE III.}

LES FONGTIONS A GROISSANGE IRRÉGULIÈRE.

Liimportance des fonctions à croissance irrégulière est beaucoup moins grande, au point de vue des applications, que celle des fonctions à croissance régulière : ce sont ces dernières que l'on a jusqu'ici exclusivement rencontrées. Il n'est cependant pas inutile de dire quelques mots des premières, ne serait-ce que pour montrer combien leur étude est plus compliquée et combien par suite il est désirable, lorsque qu'une fonction entière est définie par un procédé quelconque, de savoir démontrer que sa croissance est régulière.

Nous nous bornerons à énoncer des résultats; les méthodes développées dans ce Livre et dans les divers Mémoires auxquels nous avons renvoyé fournissent aisément les démonstrations.

Remarquons d'abord que l'expression croissance régulière (ou irrégulière) suppose un terme de comparaison supposé régulier par définition : ce terme de comparaison est la fonction exponentielle et les fonctions qui s'en déduisent par des combinaisons simples. A priori, on aurait pu prendre tel autre terme de comparaison que l'on aurait voulu, par exemple la fonction $\varpi(x)$. dont il sera question tout à l'heure. Deux raisons cependant pouvaient pousser à choisir la fonction exponentielle : d'abord les relations simples avec la suite naturelle des nombres entiers des coefficients de son développement de Taylor et des racines de l'équation obtenue en l'égalant à une constante; ensuite le fait que c'est la fonction entière la plus simple dépourvue de zéros (1). Aussi ne doit-on pas être

( $\left.{ }^{1}\right)$ On peut remarquer que cette dernière propriété conduirait nécessairement à considérer la fonction $e^{z}$, en partant de la théorie purement géométrique des fonctions analytiques (définies comme réalisant une représentation conforme). Si l'on se place à ce point de vue, on peut. remarquer que les notions du déplacement dans le plan et de l'égalité des angles, données a priori, suffisent pour définir $e^{z}$ comme fonction entière dépourvue de zéro, sans qu'il soit nécessaire d'avoir la notion de nombre entier. Dès lors, si l'on suppose que l'on a la notion de nombre ordinal, mais non celle de nombre cardinal, cette dernière s'imposera nécessairement lorsque l'on étudiera la distribution dans le plan des zéros de la fonction $e^{z}-c$. Ce fait nous a paru assez curieux pour mériter d'ètre mentionné en passant. 
étonné que ce choix se trouve justifié a posteriori par l'étude des applications.

Nous nous borncrons aux fonctions de genre fini; un principe analogue à celui de l'homogénéité du continu montrerait aisément que c'est là une restriction sans grande importance; les difficultés réelles sont aussi considérables, si l'on veut aller au fond des choses.

Une première remarque intéressante est la suivante: l'irrégularité de la croissance du module maximum $M(r)$ d'une fonction entière ne peut pas être quelconque. On verra très aisément que l'on peut trouver une fonction positive croissante $\theta(r)$ inférieure à $e^{r}$ pour toute valeur de $r$, et dont la dérivée $\theta^{\prime}(r)$, bien que toujours continue, soit, pour une infinité de valeurs de $r$ croissant indéfiniment, supérieure à toute fonction positive croissante donnée à l'avance. Lorsque l'on donne nne telle fonction $\theta(r)$, il n'existe pas de fonction entière telle que $\mathbf{M}(r)=\theta(r)$. Il y aurait lieu de rechercher, étant donnée une fonction positive croissante $\theta(r)$, quelles conditions elle doit remplir pour qu'il existe une fonction entière telle que son module maximum $M(r)$ soit du même ordre de grandeur (1) que $\theta(r)$.

Il est cependant possible de former des fonctions entières dont la croissance présente des irrégularités considérables. Indiquons, par exemple, comment on formera une fonction $\varpi(z)$ dont le module maximum $M(r)$ soit, dans une infinité d'intervalles d'étendue aussi grande qu'on veut, très voisin de $e^{r}$ et, dans une infinité d'intervalles d'étendue aussi grande qu'on veut, très voisin de $e^{r^{2}}$.

Désignons par $\varphi(x)$ une fonction positive croissante assujettie à la seule condition de croître plus vite que $e^{e^{x}}$; on peut même prendre si l'on veut $\varphi(x)=e^{e^{x}}$. Posons

$$
\begin{aligned}
& e^{z}=\Sigma a_{n} z^{n}, \\
& e^{z^{2}}=\Sigma b_{n} z^{n}
\end{aligned}
$$

et définissons les constantes $c_{n}$ par les conditions suivantes, dans lesquelles $k$ est un entier quelconque :

$$
\begin{array}{ll}
\varphi(2 k) \leqq n<\varphi(2 k+1) ; & c_{n}=a_{n}, \\
\varphi(2 k+1) \leqq n<\varphi(2 k+2) ; & c_{n}=b_{n} .
\end{array}
$$

En posant

$$
\varpi(z)=\Sigma c_{n} z^{n},
$$

la fonction $\varpi(z)$ satisfait, ainsi que toutes ses dérivées, aux conditions indiquées plus haut; il existe une intinité d'intervalles, que nous appelle-

( $\left.{ }^{1}\right)$ On entendra, par exemple, par là, que quelque petit que sojt le nombre $\varepsilon$ donné d'avance, on a, pourvu que $r$ soit assez grand,

$$
[\mathbf{M}(r)]^{1-\varepsilon}<\theta(r)<[M(r)]^{1+\varepsilon} .
$$

Cette définition pourrait d'ailleurs ètre variée suivant les besoins des applieations. 
rons intervalles de première espèce, et dont l'étendue croît indéfiniment, dans lesquels la fonction $M(r)$ correspondant à $\sigma(z)$ est inférieure à $e^{r^{1+\varepsilon}}, \varepsilon$ étant un nombre arbitrairement petit donné d'avance; il existe de même une infinité d'intervalles de seconde espèce, dans lesquels $M(r)$ est inférieur à $e^{r^{2-\varepsilon}}$.

D'après les conventions antérieurcs on doit dire que l'ordre apparent de $\varpi(z)$ est égal à deux; si l'on voulait exprimer les propriétés que nous venons d'énoncer on pourrait ajouter que cet ordre apparent est indéterminé entre un et deux, la loi de l'alternance étant réglée par la fonction $\varphi(x)$. Mais nous indiquons seulement, en passant, cette manière de s'exprimer, qu'il ne paraît pas utile d'introduire au moins pour le moment.

Relativement aux zéros de $\sigma(z)$, il résulte de ce qui précède que leurs modules forment une suite dont l'exposant de convergence cst égal à deux ; si l'on désigne par $r_{n}$ le module du $n^{\text {ième }}$ zéro, l'ordre d'infinitude de $r_{n}$ n'est pas déterminé, il est compris entre 1 et $\frac{1}{2}$; dans les intervalles de première espèce $r_{n}$ est supérieur à $n^{1-\varepsilon}$ et dans les intervalles de seconde espèce $r_{n}$ est inférieur à $n^{\frac{1}{2}+\varepsilon}$.

On démontrera aussi pour $\varpi(z)$, une fois ses zéros connus, un théorème analogue au second théorème de M. Hadamard; dans les intervalles de première espèce on trouvera des couronnes à l'intérieur desquelles le module de la fonction est supérieur à $e^{-r^{1+\varepsilon}}$ et dans les intervalles de seconde espèce des couronnes à l'intérieur desquelles le module de la fonction est supérieur à $e^{-r^{2+\varepsilon}}$.

On prouvera ainsi que l'on peut trouver une infinité de cercles de rayons indéfiniment croissants sur lesquels le rapport $\frac{\varpi^{\prime}(z)}{\tilde{\sigma}(z)}$ est aussi petit que l'on veut; on ne peut pas conclure de là que la fonction est de genre un, comme il pourrait sembler que certains auteurs l'ont pensé.

Nous arrètons là ces remarques que l'on pourrait multiplier aisément; on constatera aussi sans peine que le procédé qui nous a permis de former la fonction $o(z)$ est d'un emploi très général et qu'il est possible, par suite, de former des fonctions entières à croissance extrèmement irrégulière et s'éloignant de plus en plus du type exponentiel.

Il n'est cependant pas inutile d'observer que, en fait, les modes de croissance seront toujours liès à celui de la fonction exponentielle. En effet, nous ne connaissons primitivement que des fonctions dont le mode de croissance est exponentiel; si nous choisissons deux d'entre elles, par exemple $e^{z}$ et $e^{z^{2}}$, nous pourrons en les combinant comme plus haut en déduire une fonction irrégulière $\varpi(z)$. Mais la loi de l'alternance est nécessairement définie par une fonction croissante, que nous avons appelée $\varphi(x)$; nous devrons prendre pour $\varphi(x)$ une fonction à croissance exponentielle $\left({ }^{1}\right)$.

(') Nous donnons ici à ces mots un sens un peu plus général que plus haut; nous y comprenons les fonctions que l'on déduit de $\epsilon^{x}$ par l'itération. 
Nous obtenons ainsi la fonetion $\varpi(x)$ dont on peut dire qu'elle oscille suivant une loi exponentielle entre deux modes exponentiels. On peut former une infinité de fonctions de la même nature que $\sigma(x)$ et les combiner entre elles pour former des fonctions plus compliquées : on s'éloignera de plus en plus des types exponentiels, mais les fonctions obtenues pourront toujours être rattachées à ce type par des détours plus ou moins compliqués; elles n'en différeront pas complètement.

D'autre part, si l'on définit une fonction entière, soit par la loi de ses coefficients, soit par celle de ses zéros, on obtiendra les types de croissance analogues à ceux d'où l'on sera parti, C'est donc une question qui se pose de savoir s'il est possible de definir ${ }^{(1)}$ une fonction entière dont le type de croissance soit entièrement distinct du type exponentiel.

On pourrait songer, pour cela, à des lois arithmétiques; une des premières idées qui se présente consiste à utiliser la suite des nombres premiers; mais il résulte des recherches de Riemann que la distribution de ces nombres est intimement liée à une fonction entière $\xi(t)$ qui appartient au type exponentiel. Ce n'est donc pas là que nous pouvons trouver des types nouveaux, au moins en nous bornant aux premières approximations. Mais nous devons nous contenter de ces indications sur cette importante question des types de croissance, sur laquelle nous espérons pouvoir revenir un jour.

( ${ }^{1}$ ) Nous disons qu'une fonction est définie lorsque sa valeur numérique, pour une valeur donnée de $z$, peut itre calculée avec une approximation donnée d'avance. 


\section{TABLE DES MATIÈRES.}

Phe

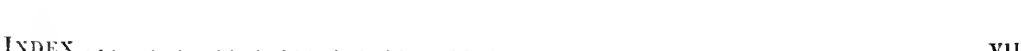

Ch.sptre I. -- Le theorème fondamental de Weierstrass............. I

Généralités sur les fonctions entières .................. I

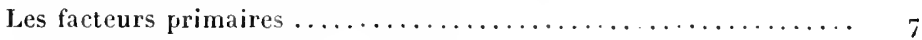

Quelques remarques sur les séries à termes positifs ........... ${ }_{\text {I }} 5$

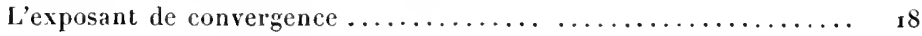

Chapitre II. - Les idees de Laguerre....................... 24

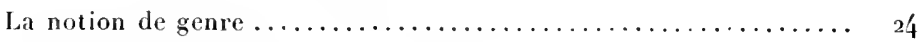

Les fonctions de genre zéro et de genre un............... 29

Les fonctions de genre fini ........................ 36

Chapitre III. - Les inégalités de $\boldsymbol{M}$. Poincaré................. 48

Le Mémoire de $\mathbf{M}$. Poincaré......................... 48

Le module maximum des fonctions d'ordre p.............. 56

Le module maximum et la fonction majorante $\ldots \ldots \ldots \ldots \ldots \ldots, 62$

Chapitre IV. - Les resultats de H. Hadamard............... ir

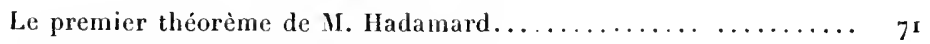

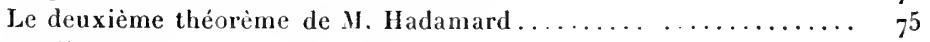

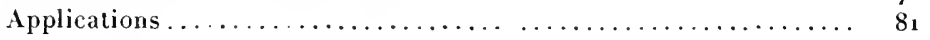

Cilapitre V. - Le théorème de. . Picard ..................... 88

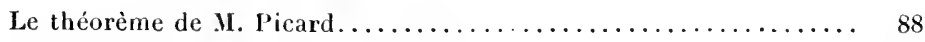

Le théorème de M. Picard généralisé ................... 94

NOTES.

Note I. - Démonstration élémentaire d'un théorème de M. Picard sur

les fonctions entieres............................... 103 
Note II. - Les fonctions à croissance régulière

Définitions et énoncés........................ ro7

Le premier théorème.......................... rog

Le deuxième théorème $\ldots \ldots \ldots \ldots \ldots \ldots \ldots \ldots \ldots \ldots \ldots \ldots \ldots \ldots$ r

Conclusion...................... 6

Note III. - Les fonctions à croissance irégulière.............. . I19

FIN DE LA TABLE DES MATIÈRES. 






\section{PLEASE DO NOT REMOVE CARDS OR SLIPS FROM THIS POCKET}

\section{UNIVERSITY OF TORONTO LIBRARY}

QA

Borel, Émile Félix Édouard

351 Justin

Leçons sur les fonctions

B67 entières

Physical \&

Applied Sci. 
